\title{
Multiparty COMMUNICATION COMPLEXITY
}

\author{
by
}

\author{
Matei David
}

A thesis submitted in conformity with the requirements for the degree of Doctor of Philosophy Graduate Department of Computer Science University of Toronto

Copyright $@ 2010$ by Matei David 


\author{
Abstract \\ Multiparty Communication Complexity \\ Matei David \\ Doctor of Philosophy \\ Graduate Department of Computer Science \\ University of Toronto
}

2010

Communication complexity is an area of complexity theory that studies an abstract model of computation called a communication protocol. In a $k$-player communication protocol, an input to a known function is partitioned into $k$ pieces of $n$ bits each, and each piece is assigned to one of the players in the protocol. The goal of the players is to evaluate the function on the distributed input by using as little communication as possible. In a Number-On-Forehead (NOF) protocol, the input piece assigned to each player is metaphorically placed on that player's forehead, so that each player sees everyone else's input but its own. In a Number-In-Hand (NIH) protocol, the piece assigned to each player is seen only by that player. Overall, the study of communication protocols has been used to obtain lower bounds and impossibility results for a wide variety of other models of computation.

Two of the main contributions presented in this thesis are negative results on the NOF model of communication, identifying limitations of NOF protocols. Together, these results consitute stepping stones towards a better fundamental understanding of this model. As the first contribution, we show that randomized NOF protocols are exponentially more powerful than deterministic NOF protocols, as long as $k \leq n^{c}$ for some constant $c$. As the second contribution, we show that nondeterministic NOF protocols are exponentially more powerful than randomized NOF protocols, as long as $k \leq \delta \cdot \log n$ for some constant $\delta<1$. 
For the third major contribution, we turn to the NIH model and we present a positive result. Informally, we show that a NIH communication protocol for a function $f$ can simulate a Stack Machine (a Turing Machine augmented with a stack) for a related function $F$, consisting of several instances of $f$ bundled together. Using this simulation and known communication complexity lower bounds, we obtain the first known (space vs. number of passes) trade-off lower bounds for Stack Machines. 


\section{Dedication}

To C.C. 


\section{Acknowledgements}

I would like to thank the members of my doctoral examination committee, including

Stephen Cook, Faith Ellen, Charles Rackoff, and Ran Raz, for their prompt reading of this thesis and for their constructive comments and suggestions.

I would like to thank Paul Beame, Periklis Papakonstantinou, Emanuele Viola, and Philipp Woelfel, for the fruitful collaboration that led to some of the results presented in this thesis.

I am especially grateful to my supervisor, Toniann Pitassi, for her guidance and support, in both professional and personal matters, throughout my doctoral studies.

Last but not least, I would like to thank my family, friends, and colleagues, for the balance they brought over the years. 


\section{Contents}

1 Introduction 1

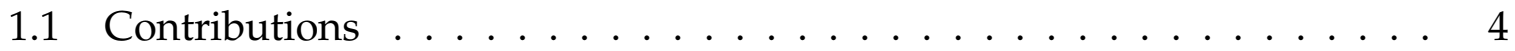

1.1.1 Separations Between Communication Complexity Classes . . . . 4

1.1.2 A New Application For Communication Complexity . . . . . . 8

1.2 Organization . . . . . . . . . . . . . . . 12

2 Preliminaries 13

2.1 Definitions . . . . . . . . . . . . . . . . 13

2.2 Some Known Facts . . . . . . . . . . . . . . . . . . . . . 20

2.2 .1 Covers and Partitions . . . . . . . . . . . . . . . 20

2.2.2 Relations Between Complexity Measures . . . . . . . . . . . . 20

2.2.3 The Discrepancy Method . . . . . . . . . . . . . . . . . 21

2.3 Important Functions . . . . . . . . . . . . . . . . . . . 23

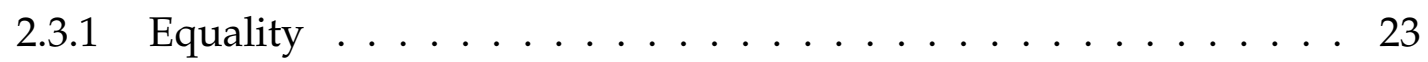

2.3 .2 Set Intersection . . . . . . . . . . . . . . . . . . . . . . 24

2.3 .3 Inner Product . . . . . . . . . . . . . . . . 26

3 Deterministic vs. Randomized Complexity 28

3.1 Special Functions and Protocols . . . . . . . . . . . . . . . . . . 30

3.2 An Alternative Characterization $\ldots \ldots \ldots \ldots$

3.3 A Lower Bound for Deterministic Protocols . . . . . . . . . . . . . . . . 35 
3.4 Public vs. Private Coins . . . . . . . . . . . . . . . . 37

3.5 Explicit Functions . . . . . . . . . . . . . . . . . . . . . . . . . 39

3.5.1 A Function Family for Three Players . . . . . . . . . . . . . 40

3.5.2 A Function Family for Three or More Players . . . . . . . . . . 48

3.6 Discussion . . . . . . . . . . . . . . . . . . . 51

4 Randomized vs. Nondeterministic Complexity $\quad 54$

4.1 Preliminaries. . . . . . . . . . . . . . . . . 58

$4.1 .1 \quad$ Lift Function . . . . . . . . . . . . . . . . . . . . . 58

4.1 .2 Correlation . . . . . . . . . . . . . . . . . . . . 59

4.1 .3 Approximate Degree . . . . . . . . . . . . . . . . . . . . 61

4.2 Non-explicit Separation . . . . . . . . . . . . . . . . . . . 62

4.3 Explicit Separation . . . . . . . . . . . . . . . . 73

4.4 Discussion . . . . . . . . . . . . . . . . . . . . . 79

5 Trade-off Lower Bounds for Stack Machines $\quad 82$

5.1 Outline of the Argument . . . . . . . . . . . . . . . 85

5.2 Preliminaries . . . . . . . . . . . . . . . . . 95

5.3 The Reduction . . . . . . . . . . . . . . . . . . . . . . . . . . 102

5.4 Bounding the Number of Corrupted Instances $\ldots \ldots \ldots$

5.5 Simulating a Stack Machine with a Protocol . . . . . . . . . . . . . 108

5.6 Consequences of the Reduction . . . . . . . . . . . . . . . 116

5.7 Discussion . . . . . . . . . . . . . . . . . 123

$\begin{array}{ll}\text { Bibliography } & 125\end{array}$ 


\section{Chapter 1}

\section{Introduction}

Communication complexity is an area of complexity theory whose object of study is the amount of communication that is needed in order to evaluate a function when its input is split among several computationally unbounded players. This question has been shown to have applications in a wide variety of other areas of theoretical computer science, such as: cell probe complexity, VLSI circuit design, Turing Machine and circuit complexity, pseudorandomness, proof complexity, and streaming. The communication model was initially designed as a theoretical tool for deriving lower bounds in other established models of computation via reductions. However, in time, the proliferation of such types of connections has dramatically increased the appeal of purely communication complexity theoretic results. Of the three major contributions presented in this work, two are of the latter type, answering fundamental questions about communication complexity, and the third is of the former type, providing new trade-off lower bounds for the computational power of Turing Machines equipped with an unbounded stack.

Communication complexity itself can be seen as the study of an abstract model of computation, called a communication protocol. Let $k$ denote the number of players, let 
$n$ denote the input size, 1 and let $f:\{0,1\}^{k n} \rightarrow Z$ be a function, for some range $Z$. An input $x$ to $f$ is split into $k$ parts of $n$ bits each, and every part is "given" to one of the $k$ players in one the two ways described below. Either way, none of the players can see the entire input $x$. Still, their common goal is to evaluate $f$ on $x$, and to that end, they "communicate" with each other. A communication protocol specifies the order in which the players communicate, as well as the content of what they communicate. We assume that the players act collaboratively, in that they do not deviate from the protocol agreed upon in advance. We say that a protocol $P$ computes the function $f$ if, for every input $x$, all players eventually learn $f(x)$ after following $P$. The cost of a communication protocol is the maximum amount of communication, over all inputs. The communication complexity of a function is the minimum cost of a protocol computing it. In general, we allow the number of players $k$ to be a function of the input size $n$, and we are interested in the asymptotic behaviour of the cost of a protocol, as a function of $n$.

We consider two types of communication protocols. In a number-in-hand (NIH) protocol, the part of the input given to each player is private to that player. Thus, every player gets to see $n$ of the $k \cdot n$ bits of $x$. In contrast, in a number-on-forehead (NOF) protocol, the part of the input given to each player is metaphorically placed on that player's forehead, so that each player sees everybody else's input but its own. Thus, every player gets to see $(k-1) \cdot n$ of the $k \cdot n$ bits of $x$. Communication complexity was first introduced by [Yao79] in the case of $k=2$ players, where the NIH and NOF models coincide. We see that, as the number of players $k$ grows with the input size $n$, the two models behave quite differently.

In the NIH model, each player sees a $1 / k$ fraction of the $k \cdot n$ input bits, so computing a function becomes harder as $k$ grows. Lower bounds for the communication com-

\footnotetext{
${ }^{1}$ In fact, $n$ is the number of bits given to each player, and $k \cdot n$ is the size of the input to the function. We discuss the difference between these measures in Section 2.1.
} 
plexity of functions in the case of $k=2$ players usually transfer to the NIH model for more players. There are, however, settings in which computation in the NIH model becomes easier as $k$ grows. Specifically, this is the case for certain promise functions, notably the promise Set Intersection function (discussed in Section 2.3), where a communication protocol is free to output anything on inputs which do not satisfy a certain condition. The NIH model has received increased attention after [AMS99] used it to obtain lower bounds for streaming algorithms.

The NOF model was introduced by [CFL83]. In this model, each player sees a $(k-1) / k$ fraction of all input bits, so computing a function becomes easier as $k$ grows. [HG91, BT94] show that proving strong lower bounds for the communication complexity of a function in the randomized NOF model with polylogarithmically $\left((\log n)^{\Theta(1)}\right)$ many players would answer a major open problem in circuit complexity. The best lower bounds that we know in this model are based on the discrepancy method [BNS92], and they all become trivial as soon as $k$, the number of players, is greater than or equal to the logarithm (base 2) of $n$, the number of bits assigned to each player.

An important underlying assumption in communication complexity is that the individual players participating in a protocol are computationally unbounded. That is, what a player communicates is an arbitrary function of the information available to that player: the part of the input the player sees and the communication that occurred so far. The motivation for this assumption is two-fold. On the one hand, by removing any (time, space) limits on the classical computational power of individual players, we are avoiding a return to classical complexity questions. On the other, this assumption makes communication protocols very powerful: two players can solve the Halting problem by simply having one send over its share of the input to the other, at which point the latter sees the entire input and can simply announce the value of the function. Since we are interested in using communication complexity for deriving lower 
bounds in other models of computation via reductions, making the communication model stronger can only widen the applicability of communication complexity results.

\subsection{Contributions}

In this section, we informally present the major contributions of this work. We defer the formal statements of these results to their respective Chapters.

\subsubsection{Separations Between Communication Complexity Classes}

As in the case of other models of computation, we can consider deterministic, randomized, and nondeterministic communication protocols. [BFS86] first studied communication complexity classes in the case of $k=2$ players. Motivated by the fact that the communication complexity of every function is at most linear in this case, they proposed that a communication protocol should be called "efficient" when it has at most polylogarithmic $\left((\log n)^{O(1)}\right)$ cost. Equipped with a notion of efficiency, one can define the corresponding classes (e.g., $\mathrm{P}^{\mathrm{cc}}, \mathrm{BPP}^{\mathrm{cc}}, \mathrm{NP}^{\mathrm{cc}}$ ) of functions which have efficient protocols of the type classically associated with that class. A fundamental problem in communication complexity is understanding the respective powers of these types of protocols, and, in particular, separating the corresponding complexity classes. While in the case of $k=2$ players (and in the NIH model in general), the answer to this problem was fairly well understood, much less was known about the NOF model for $k>2$ players. Two of the major results we present in this work are exponential separations between the powers of various types of NOF protocols.

Deterministic vs. Randomized NOF Protocols. A deterministic protocol must be correct on every input, whereas a randomized protocol has access to a random string and is allowed to err on every input with a small constant probability. The question of 
whether randomized polynomial time computation (i.e., BPP) is more powerful than deterministic polynomial time computation (i.e., P) is a long standing open problem in classical computational complexity.

It turns out that, in the context of $k=2$ player communication complexity, this question has a rather simple affirmative answer (see the discussion of the Equality function in Section 2.3). However, no similar answer was known in the NOF model prior to the results presented in this work. Intuitively, the difficulty in proving such a separation was that essentially the only method we knew for deriving strong lower bounds for NOF deterministic protocols also derives lower bounds for NOF randomized protocols [BNS92].

As the first major contribution of this work, we present the first (and to this date, the only) exponential separation $\left(n^{\Omega(1)}\right.$ vs. $\left.(\log n)^{O(1)}\right)$ between the powers of deterministic and randomized $k$-player NOF communication protocols, which holds as long as the number of players grows at most as a polynomial in the input size, that is, $k \leq n^{O(1)}$. We point out that the dependency of the lower bound for deterministic protocols on the number of players is exponentially better than that of all known lower bounds for randomized protocols, which become trivial when $k \geq \log n$.

Informally, to prove this result, we consider a class of special functions that have the following two properties: on the one hand, every function has an efficient randomized protocol; on the other, every efficient deterministic protocol for such a function has a small encoding. We are then able to apply a counting argument, showing that there are more functions in this class than there are encodings of efficient deterministic protocols for them.

It is natural to ask what is the "simplest" function that achieves this separation, in the sense of its classical time or space complexity. Unfortunately, the smallest computational complexity class in which we can currently place the separating function is doubly-exponential time (i.e., DTIME $\left(2^{2^{n}}\right)$ ). It would be interesting to find such a 
function in a smaller class. To this end, we propose a more natural candidate function that, we believe, might achieve this separation.

It is standard to consider two types of randomized communication protocols: in public-coin protocols the random string is shared by all players, whereas in privatecoin protocols each player has its own random string. Newman shows that for every function, its public-coin and private-coin randomized communication complexities are separated by at most an additive $O(\log n)$ term [New91]. As a corollary of the exponential separation between deterministic and randomized NOF protocols, we also obtain an optimal separation $(\Theta(\log n)$ vs. $O(1))$ between the powers of private-coin and public-coin randomized $k$-player NOF communication protocols, as long as $k \leq n^{c}$ for some constant $c<1$.

This work appears in [BDPW07].

Randomized vs. Nondeterministic NOF Protocols. One way to look at the difference between one-sided error randomized and nondeterministic protocols for a boolean function is through their correctness condition: both types of protocols have access to a random string; both are required to be correct on every 0 input of the function; on every 1 input, however, a randomized protocol must be correct with probability greater than a constant, whereas a nondeterministic protocol need only be correct with probability greater than 0 . Clearly, nondeterministic protocols are at least as powerful as one-sided error randomized protocols. Moreover, general (two-sided error) randomized protocols are allowed to err with constant probability on 0 inputs as well, so they are a priori incomparable in power to nondeterministic protocols. Classifying the relative powers of nondeterministic polynomial time computation (i.e., NP) and randomized polynomial time computation (i.e., RP and BPP) are long standing open problems in classical computational complexity.

In the context of $k=2$ player communication complexity, these questions are 
solved: we know that nondeterminism is more powerful than one-sided error randomization, and that nondeterminism and two-sided error randomization are incomparable (see the discussion in Section 2.3). Until recently, these questions have been open in the NOF model.

As in the case of determinism and randomization, the difficulty in obtaining such separation results was that the only lower bound method we knew for NOF randomized protocols, the so-called discrepancy method [BNS92], would in fact prove lower bounds even for NOF nondeterministic protocols. This difficulty was addressed by [Kla07, Raz03], who propose a general framework, called the generalized discrepancy method, which could potentially be used to prove lower bounds for randomized protocols without deriving lower bounds for nondeterministic protocols. A series of relatively recent results was initiated by [She09, She08b], who obtained communication lower bounds by studying the representation of boolean function by real polynomials. Building on this work, [LS08] and [CA08] developed a way to apply the generalized discrepancy method in order to prove lower bounds for the Set Intersection function, and they presented an exponential separation between the powers of randomized and nondeterministic $k$-player NOF communication protocols, when the number of players satisfies $k<\log \log n$.

As the second major contribution of this work, we extend the results above, and we present an exponential separation $\left(n^{\Omega(1)}\right.$ vs. $\left.(\log n)^{O(1)}\right)$ between the powers of randomized and nondeterministic $k$-player NOF communication protocols, for every constant $\delta<1$ and for every function $k=k(n)$ that satisfies $k<\delta \cdot \log n$ for all sufficiently large $n$. The dependency of this separation on the number of players is state-of-the-art: it is a major open problem in communication complexity to obtain any nontrivial lower bound for $k$-player randomized NOF protocols when $k \geq \log n$.

We obtain this separation by applying the generalized discrepancy method to a "lifted function", which is constructed from a "base function" and a "selection func- 
tion". In the case of [LS08, CA08], the separation between randomized and nondeterministic protocols is achieved by the Set Intersection function, which is itself a lifted function, with base function Or and selection function And. In our case, we observe that as long as the base function is Or, no matter what selection function we use, the resulting lifted function has an efficient nondeterministic protocol. Moreover, we show that if we choose a selection function at random, the resulting lifted function has no efficient randomized protocol. Using the probabilistic method, we conclude that there is some selection function for which the resulting lifted function separates randomized and nondeterministic protocols. Intuitively, our arguments are simpler and we achieve a better dependency on the number of players $(k<\delta \cdot \log n$ as opposed to $k<\log \log n$ in [LS08, CA08]) because of the advantage we gain by using a random selection function instead of the structured And function. We are subsequently able to derandomize the argument using a result of [NN93], obtaining a polynomial time computable function that achieves the same separation.

This work appears in [DPV09].

Subsequent to and building on our work, [BHN09] proved that a separation between randomized and nondeterministic protocols can also be achieved by a function computable by small depth $\left(\mathrm{AC}_{4}^{0}\right)$ circuits, albeit they can only handle $k \leq \delta \cdot \log n$ players, for some constant $\delta<1$, rather than for every $\delta<1$ in our case. They also improve the dependency on the number of players of the randomized lower bound for the Set Intersection function from $k<\log \log n$ in [LS08, CA08] to $k<O\left((\log n)^{1 / 3}\right)$.

\subsubsection{A New Application For Communication Complexity}

Understanding the computational power of Turing Machines and circuits in general, and understanding "efficient" computation in particular, are some of the most established goals of theoretical computer science. Consider the classes P and L, of languages decided by deterministic Turing Machines in polynomial time and logarithmic 
space, respectively. For every integer $i \geq 0$, let NC ${ }^{i}$ denote the class of languages decided by circuits ${ }^{2}$ of polynomial size, depth $O\left((\log n)^{i}\right)$, consisting of bounded fan-in And, Or and Not gates. In general, we regard $P$ and $L$ as modelling efficient timebounded and space-bounded computation, respectively, and we regard the classes $N C^{i}$ as modelling efficient parallelizable computation. We know that

$$
N C^{0} \subsetneq N C^{1} \subseteq \mathrm{L} \subseteq N C^{2} \subseteq \ldots \subseteq N C^{i} \subseteq \ldots \subseteq \mathrm{P},
$$

but we don't know whether any of the inclusions beyond $N C^{1}$ are proper.

One possible way to tackle the problem of separating complexity classes defined using different models of computations (e.g., Turing Machines, combinatorial circuits) and different resource bounds (e.g., time, space, size, depth) is to rephrase them as separations of classes based a common computational model, a common resource bound, and prove lower bounds for that common resource. For the complexity classes above, such a line of attack was initiated by [Coo71]. A stack, also called a push-down storage, is a model for a possibly unbounded storage space which comes with a First-In LastOut access restriction. A Stack Machine is a classical Turing Machine equipped with an unbounded stack. ${ }^{3}$ [Coo71] gave a fascinating characterization of a time-bounded complexity class in terms of a space-bounded computational model, showing that the class of languages decided by logarithmic space Stack Machines exactly equals P. It is not hard to see that there is no loss in computational power in assuming that a logarithmic space Stack Machine also operates in at most exponential $\left(2^{\mathrm{O}(n)}\right)$ time. Moreover, in a series of subsequent results [Ruz80, Ruz81, $\left.\mathrm{BCD}^{+} 89, \mathrm{All} 89\right]$, a surprising connection was established between space- and time- bounded Stack Machines and combinatorial circuits. Denoting by $\mathrm{SM}(s, t)$ the class of languages decided by deterministic Stack Machines operating in space $s$ and time $t$, it was shown that, for every

\footnotetext{
${ }^{2}$ We are referring to logarithmic space uniform circuits in this case.

${ }^{3}$ This model of computation is also called an AuxPDA.
} 
integer $i \geq 1$,

$$
\mathrm{NC}^{i} \subseteq \mathrm{SM}\left(\mathrm{O}(\log n), 2^{O\left((\log n)^{i}\right)}\right) \subseteq \mathrm{NC}^{i+1} \subseteq \ldots \subseteq \mathrm{P}=\mathrm{SM}\left(\mathrm{O}(\log n), 2^{O(n)}\right)
$$

Thus, separations between such classes as $L, N C^{i}$, and $P$, can be achieved by proving time lower bounds for logarithmic space Stack Machines. While it is not clear how to prove such lower bounds, it is still useful to have different avenues for attacking computational class separations.

Trade-off Lower Bounds for Stack Machines. In spite of the connections laid out above between Stack Machines and major open problems in computational complexity, this model is not well understood.

As the third major contribution of this work, we provide the first unconditional lower bounds for Stack Machines. We do not know how to tackle time lower bounds directly. Instead, we consider the number of passes a Stack Machine makes over its input. We do not lose generality in assuming that in every pass, the input head moves from one end of the tape to the other. Sometimes this measure is also called reversal complexity, as the number of passes equals one plus the number of reversals. In this work, we prove the first unconditional lower bounds for the number of passes a logarithmic space Stack Machine makes on its input tape. Specifically, we give two examples of functions for which any logarithmic space Stack Machine requires a sub-linear polynomial $\left(\Omega\left(n^{\beta}\right)\right.$ for some $\left.\beta<1\right)$ number of passes.

Clearly, the number of passes is also a lower bound on the number of transitions of a Stack Machine, and hence, on its time. Unfortunately however, our method comes with an important limitation. Specifically, by using a reduction to communication complexity it is not clear how to obtain any super-linear lower bounds, because, as we discuss in Section 2.1, the communication complexity of any function is at most linear. Still, it is our hope that these results would further the understanding of the Stack Machine model of computation. 
Our technical contribution comes in the form of a reduction, showing that we can use known communication complexity lower bounds to derive trade-off lower bounds between the space and the number of passes required by a Stack Machine. Informally speaking, we show that for every "base function" $f$, we can define a "lifted function" $F$, consisting of a number of instances of $f$ applied on a larger input and combined together, such that the following holds: given an efficient (in terms of space and passes) Stack Machine for $F$, we can construct an efficient communication protocol for $f$. Applying this construction to base functions $f$ which are known to be hard in the communication complexity model yields functions $F$ which are hard for Stack Machines.

This type of reduction, connecting efficient communication protocols and space bounded computation, is not new. One of the first examples is [BNS92], which derived time-space trade-offs for multi-head Turing Machines. Subsequently, such reductions have been used to derive lower bounds in streaming [AMS99], an area of computer science whose object of study is the power of Turing Machines with small space and a single (or, very few) pass(es) over the input tape. The method by which we obtain our results is inspired by [BHN08], which gave similar trade-off lower bounds, between space and number of passes, for Turing Machines that have access to $a$ constant number of "external" tapes, and where passes are counted over all of these tapes. Instead, in our model, the Turing Machine has access to an unbounded stack. To the best of our knowledge, these models are incomparable [DPS09]. It is the treatment of the stack that constitutes the main technical novelty in our result. To put this into perspective, observe that a Turing Machine equipped with two unbounded stacks can decide any decidable language, using no workspace and a single pass over the input. 


\subsection{Organization}

In Chapter 2, we give formal definitions for the communication complexity model, and we present several important background results. The following three Chapters correspond to the three major contributions of this work. In Chapter 3 , we give the separation between deterministic and randomized NOF communication protocols. In Chapter 4 , we give the separation between randomized and nondeterministic NOF communication protocols. In Chapter 5, we give the reduction connecting Stack Machines and NIH communication protocols. Additional definitions and related work are presented in each of these three Chapters. 


\section{Chapter 2}

\section{Preliminaries}

We begin in Section 2.1 with the communication complexity notation, definitions, and terminology that will be used throughout this work. Additional definitions pertaining to specific chapters will be introduced at the beginning of those chapters. In Section 2.2, we survey some relevant known facts about communication complexity measures. Finally, in Section 2.3, we introduce some of the important functions that have been studied in the context of communication complexity.

\subsection{Definitions}

General Notation Let $\mathbb{N}=\{1, \ldots\}$ denote the set of positive integers, and let $\mathbb{R}$ denote the set of real numbers. For $a \in \mathbb{N}$, we write $[a]$ for $\{1, \ldots, a\}$. We write $\log$ for $\log _{2}$

Whenever we define an integer quantity using a real-valued expression, we are implicitly taking the floor of that expression. For example, if $m$ must be an integer, when we write $m:=n^{1 / 2}$, we mean $m:=\left\lfloor n^{1 / 2}\right\rfloor$.

We use the standard notation $O(\cdot), o(\cdot), \Omega(\cdot), \omega(\cdot)$, and $\Theta(\cdot)$, to make statements about the asymptotic growth of functions. We combine asymptotic notation with other 
functions in the standard way. Thus, for example, for functions $f, g$ of a common parameter $n$, and for an increasing positive function $h$ :

- " $f=h(O(g))$ " or " $f \leq h(O(g))$ " means that there exist constants $c$ and $n_{0}$ such that for all $n \geq n_{0}, f(n) \leq h(c \cdot g(n))$;

- " $f=h(\Omega(g))$ " and " $f \geq h(\Omega(g))$ " have a similar meaning, except that the inequality is now $\geq$ instead of $\leq$;

- " $f=h(\Theta(g))$ " means that there exist constants $c_{1}, c_{2}$ and $n_{0}$ such that for all $n \geq n_{0}, h\left(c_{1} \cdot g(n)\right) \leq f(n) \leq h\left(c_{2} \cdot g(n)\right)$.

Communication Protocols [KN97] is a good communication complexity reference, even though it largely focuses on the case of only $k=2$ players. We adapt the notation and terminology in here.

In general, we use $k$ to denote the number of players in a communication protocol. Let $n$ denote the number of bits "assigned" to each player (in one of the ways described below). We allow $k=k(n)$ to be a non-decreasing function of $n$.

A $k$-player communication protocol over the input set $\left(\{0,1\}^{n}\right)^{k}$ operates as follows. An input $x=\left(x_{1}, \ldots, x_{k}\right) \in\left(\{0,1\}^{n}\right)^{k}$ is (conceptually) split among $k$ players in one of the following two ways:

- In a number-in-hand (NIH) protocol, player $i \in[k]$ sees only $x_{i}$.

- In a number-on-forehead (NOF) protocol, player $i \in[k]$ sees $x_{j}$, for all $j \in[k] \backslash\{i\}$.

The players communicate by writing bits on a blackboard seen by everybody. A deterministic protocol specifies:

- A function mapping every blackboard content to:

- whether or not the communication is over;

- if the communication is over, the output of the protocol (a single bit); and 
- if the communication is not over, the identity of the next player to communicate.

- For every player $i$, a function mapping the input player $i$ sees and the blackboard contents to a bit that player $i$ communicates. (This function is only used when it is the turn of player $i$ to communicate.)

The cost $c=c(n)$ of a protocol is the maximum number of bits written on the blackboard over all possible inputs.

A public-coin randomized protocol is one where the players have access to a shared uniform random string, which is not counted towards the cost of the protocol. Alternatively, one can see a public-coin randomized protocol of cost $c$ as a probability distribution over deterministic protocols of cost $c$.

A private-coin randomized protocol is one where the players each have access to a private uniform random string. Note that, in a private-coin randomized NOF protocol, each input bit is seen by many players, but each random bit is private to only one player.

Every deterministic communication protocol $P$ computes a function in the obvious way: on input $x \in\left(\{0,1\}^{n}\right)^{k}$, the players communicate as dictated by $P$, until an output is produced. We assume that protocols never run forever. Slightly overloading notation, we denote by $P(x)$ the output of $P$ on $x$. When $P$ is a randomized protocol, we write $P(x, \rho)$ for the output of $P$ on $x$ when the random string(s) in $P$ are set to $\rho$.

Communication Complexity Measures Let $f:\left(\{0,1\}^{n}\right)^{k} \rightarrow\{0,1\}$ be a boolean function. All the communication complexity measures below are defined independently for the NIH and the NOF models. When it is unclear which of the two models we are referring to, we include it as a subscript. Thus, for any of the complexity measures $C$ defined below, we usually write $C(f)$, but in full notation we write $C_{\mathrm{NIH}}(f)$ or $C_{\mathrm{NOF}}(f)$. 
The deterministic communication complexity of $f$, written $D_{k}(f)$, is the minimum cost of a $k$-player deterministic protocol for $f$ that always outputs the correct answer.

Let $\mu$ be a probability distribution over $\left(\{0,1\}^{n}\right)^{k}$, and let $0 \leq \epsilon<1$. Let $D_{k}^{\mu, \epsilon}(f)$ denote the minimum cost of a deterministic protocol for $f$ that outputs the correct answer on all but an $\epsilon$ measure of inputs, as weighted by $\mu$. Let $D_{k}^{\mu}(f):=D_{k}^{\mu, 1 / 3}(f)$. We refer to this quantity as the distributional communication complexity of $f$ under $\mu$.

For $0 \leq \epsilon<1$, we say that a (public-coin or private-coin) randomized protocol $P$ has error $\epsilon$ if, for every input, $P$ is correct with probability at least $1-\epsilon$ over the choice of the random string(s). Formally,

$$
\forall x \in\left(\{0,1\}^{n}\right)^{k}, \operatorname{Pr}_{\rho}[P(x, \rho)=f(x)] \geq 1-\epsilon .
$$

We refer to this type of error as two-sided error. Trivially, any function has a two-sided 1/2 error protocol: just output a random bit. We say that a protocol has two-sided unbounded error if $\epsilon<1 / 2$. By standard arguments, a two-sided $\epsilon$ error protocol can be repeated a constant number of times (taking the majority vote) in order to decrease the error probability below any constant, as long as $\epsilon \leq \epsilon^{\prime}$ for a constant $\epsilon^{\prime}<1 / 2$. We say that a protocol has two-sided bounded error if $\epsilon \leq 1 / 3$.

We say that $P$ has false-negatives (respectively, false-positives) error if $P$ is correct on all 0 -inputs (respectively, 1-inputs). Formally, $P$ has false-negatives error $\epsilon$ if

$$
\forall x \in\left(\{0,1\}^{n}\right)^{k},\left\{\begin{array}{l}
f(x)=0 \Rightarrow \forall \rho, P(x, \rho)=0 \\
f(x)=1 \Rightarrow \operatorname{Pr}_{\rho}[P(x, \rho)=1] \geq 1-\epsilon
\end{array}\right.
$$

We refer to either false-negatives or false-positives as one-sided error. Trivially, any function has a one-sided 1 error protocol: just output the value on which we're not allowed any error. We say that a protocol has one-sided unbounded error if $\epsilon<1$. By standard arguments, a one-sided $\epsilon$ error protocol can be repeated a constant number of times in order to decrease the error probability below any constant, as long as $\epsilon \leq \epsilon^{\prime}$ for a constant $\epsilon^{\prime}<1$. We say that a protocol has one-sided bounded error if $\epsilon \leq 1 / 2$. 
Let $R_{k}^{\epsilon}(f)$ denote the minimum cost of a private-coin randomized protocol for $f$ with (two-sided) error $\epsilon$. The private-coin randomized communication complexity of $f$ is $R_{k}(f):=R_{k}^{1 / 3}(f)$.

For $b \in\{0,1\}$, let $R_{k}^{b, \epsilon}(f)$ denote the minimum cost of a private-coin randomized protocol for $f$ with one-sided error $\epsilon$ that is correct on $b$-inputs. The private-coin false-negatives (respectively, false-positives) randomized communication complexity of $f$ is $R_{k}^{b}(f):=R_{k}^{b, 1 / 2}(f)$, where $b=1$ for false-negatives, and $b=0$ for false-positives.

The public-coin communication complexity measures $R_{k}^{\mathrm{pub}, \epsilon}(f), R_{k}^{\mathrm{pub}}(f), R_{k}^{\mathrm{pub}, b, \epsilon}(f)$, and $R_{k}^{\text {pub,b}}(f)$ are defined similarly, by considering public-coin protocols.

A nondeterministic protocol is a private-coin randomized protocol with false-negatives unbounded error. Let $N_{k}(f)$, the nondeterministic communication complexity of $f$, be the minimum cost of a nondeterministic protocol for $f$. The co-nondeterministic communication complexity $N_{k}^{0}(f)$ is defined similarly, by allowing false-positives unbounded error.

For any of these complexity measures above, we omit the subscript $k$ when it is clear from the context.

Nonuniformity and Input Size Communication complexity is a nonuniform model of computation, meaning that, when computing the functions in a certain family, we are allowed to used different protocols for different input sizes.

Properly speaking, we are interested in computing a function family $f=\left(f_{N}\right)_{N \in \mathbb{N}}$ with a protocol family $P=\left(P_{N}\right)_{N \in \mathbb{N}}$, where $P_{N}$ is used to compute $f_{N}$, and we study the growth of the cost $c=c(N)$ of $P_{N}$ as a function of $N$. We sometimes use the terms "function" and "protocol" even though we mean "function family" and "protocol family".

All communication complexity measures defined above are functions of the input size $N$. However, we find it more convenient, and quite standard, to study the growth 
of these functions in terms of $n$, the number of bits assigned to each player, instead of $N$, the total input size. We implicitly assume that $k=k(n)$ is a "well-behaved" integer function of $n$, in the sense that: it is non-decreasing, it is greater than or equal to 2 , and it doesn't grow faster than a polynomial in $n$ (i.e., $k \leq n^{O(1)}$ ).

We use the notation " $f=\left(f_{k, n}\right)_{n}$ " to denote a family of functions where for $n \in \mathbb{N}$, the corresponding function for that input size is $f_{k(n), n}$, and its domain of definition is $\left(\{0,1\}^{n}\right)^{k(n)}$. Since we are only interested in the asymptotic growth of the various communication complexities of $f$, we sometimes leave functions families undefined for small $n$. We use a similar notation for protocol families.

Communication Complexity Classes Every function has a trivial deterministic NIH protocol with cost $(k-1) \cdot n+1$ : players $1, \ldots, k-1$ each communicate their input, and player $k$ announces the output. Similarly, every function has a trivial deterministic NOF protocol with cost $n+1$ : player 1 communicates the input on the forehead of player 2, and player 2 announces the output. Motivated by these upper bounds and following [BFS86], we say that a protocol family $P=\left(P_{k, n}\right)_{n}$ is efficient if the cost $c=c(n)$ of $P_{k, n}$ grows like at most a polylogarithm, that is, $c \leq(\log n)^{O(1)}$.

Equipped with this notion, we are ready to define the communication complexity analogues of classical computational complexity classes. Let $\mathrm{P}_{k}^{\mathrm{cc}}, \mathrm{RP}_{k}^{\mathrm{cc}}, \operatorname{coRP}_{k}^{\mathrm{cc}}, \mathrm{BPP}_{k}^{\mathrm{cc}}$, $\mathrm{NP}_{k}^{c c}$, and coNP ${ }_{k}^{c c}$ denote the sets of function families $f=\left(f_{k, n}\right)_{n}$ for which $D_{k}\left(f_{k, n}\right)$, $R_{k}^{1}\left(f_{k, n}\right), R_{k}^{0}\left(f_{k, n}\right), R_{k}\left(f_{k, n}\right), N_{k}\left(f_{k, n}\right)$ and $N_{k}^{0}\left(f_{k, n}\right)$ are $(\log n)^{O(1)}$, respectively.

These classes are defined both in the NIH and the NOF models. When it is unclear which model we are referring to, we include it as a subscript. Thus, for example, we write $\mathrm{P}_{\mathrm{NIH}, k}^{\mathrm{cc}}$ and $\mathrm{P}_{\mathrm{NOF}, k}^{\mathrm{cc}}$.

Rectangles and Cylinder Intersections The following types of sets play an important role in analyzing communication protocols. A set $R \subseteq\left(\{0,1\}^{n}\right)^{k}$ is called a rectangle if there are sets $R_{1}, \ldots, R_{k}$ such that for all $i \in[k], R_{i} \subseteq\{0,1\}^{n}$, and $R=$ 
$R_{1} \times \cdots \times R_{k}$. A set $S \subseteq\left(\{0,1\}^{n}\right)^{k}$ is a cylinder in the $i$-th dimension if membership of a $k$-tuple in $S$ is not dependent on the $i$-th coordinate. A set $T \subseteq\left(\{0,1\}^{n}\right)^{k}$ is a cylinder intersection if there are sets $S_{1}, \ldots, S_{k}$ such that for all $i \in[k], S_{i} \subseteq\left(\{0,1\}^{n}\right)^{k}$ is a cylinder in the $i$-th dimension, and $T=S_{1} \cap \cdots \cap S_{k}$. Note that for $k=2$, rectangles and cylinder intersections coincide.

Discrepancy For a boolean function $f: Z \rightarrow\{0,1\}$, a set $S \subseteq Z$, and a distribution $\mu$ on $Z$, the discrepancy of $f$ on $S$ under $\mu$ is defined as the difference between the weight of the 0 s and $1 \mathrm{~s}$ of $f$ in $S$ :

$$
\operatorname{disc}_{\mu}(f, S):=\left|\mu\left(f^{-1}(0) \cap S\right)-\mu\left(f^{-1}(1) \cap S\right)\right|
$$

When $f$ is a function over the range $\{-1,+1\}$, observe that:

$$
\operatorname{disc}_{\mu}(f, S)=\underset{x \sim \mu}{\mathrm{E}}\left[f(x) \cdot 1_{S}(x)\right]
$$

where $1_{S}: Z \rightarrow\{0,1\}$ is the characteristic function of the set $S$. That is, $1_{S}(x)=1$ if and only if $x \in S$.

For a function $f:\left(\{0,1\}^{n}\right)^{k} \rightarrow\{0,1\}$ and a distribution $\mu$ on $\left(\{0,1\}^{n}\right)^{k}$, we define the NIH (respectively, NOF) discrepancy of $f$ as the maximum discrepancy of $f$ under rectangles (respectively, cylinder intersections).

$$
\begin{aligned}
\operatorname{disc}_{\mathrm{NIH}, \mu}(f) & :=\max _{\text {rectangle } R \subseteq\left(\{0,1\}^{n}\right)^{k}} \operatorname{disc}_{\mu}(f, R) \\
\operatorname{disc}_{\mathrm{NOF}, \mu}(f) & :=\operatorname{myx}_{\text {cylinder intersection } T \subseteq\left(\{0,1\}^{n}\right)^{k}} \operatorname{disc}_{\mu}(f, T)
\end{aligned}
$$

For a function $f: X \rightarrow Z$ and a set $A \subseteq X$, we say that $A$ is $f$-monochromatic if there exists $z \in Z$ such that for all $x \in A, f(x)=z$. 


\subsection{Some Known Facts}

\subsubsection{Covers and Partitions}

The following fact is an immediate consequence of the definition of a communication protocol.

Fact 2.1 (Proposition 1.14 and Lemma 6.9 in [KN97]). For every NIH (respectively, NOF) protocol over $\left(\{0,1\}^{n}\right)^{k}$, the set of inputs which lead to a certain blackboard content is a rectangle (respectively, cylinder intersection) in $\left(\{0,1\}^{n}\right)^{k}$.

Since the output of a protocol is determined by the blackboard content, we also obtain the following:

Fact 2.2 (Lemma 1.16 and Lemma 6.10 in [KN97]). Every c-bit NIH (respectively, NOF) protocol $P$ over $\left(\{0,1\}^{n}\right)^{k}$ induces a partition of the input space $\left(\{0,1\}^{n}\right)^{k}$ into at most $2^{c}$ rectangles (respectively, cylinder intersections) $S_{1}, \ldots, S_{2 c}$, such that Poutputs the same value on all inputs from the same set in the partition. That is, every set $S_{i}$ is $P$-monochromatic.

In particular, if $P$ is a deterministic protocol for $f$, then every set $S_{i}$ is $f$-monochromatic.

In the case of nondeterministic protocols, we have a similar result.

Fact 2.3 (Section 2.1 in [KN97]). Every c-bit NIH (respectively, NOF) nondeterministic protocol P for $f:\left(\{0,1\}^{n}\right)^{k} \rightarrow\{0,1\}$ induces a cover of all 1-inputs of $f$ by at most $2^{c}$ $f$-monochromatic rectangles (respectively, cylinder intersections).

\subsubsection{Relations Between Complexity Measures}

Deterministic vs. Private Coin Randomized For the case of $k=2$ players, [KN97] give the following connection between deterministic and private-coin randomized protocols. Intuitively, this is obtained by having a deterministic protocol estimate the probabilities of following certain paths in a randomized communication protocol. 
Fact 2.4 (Lemma 3.8 in [KN97]). For every function family $f=\left(f_{2, n}\right)_{n}$,

$$
R\left(f_{2, n}\right)=\Omega\left(\log D\left(f_{2, n}\right)\right)
$$

Public Coin vs. Private Coin Randomized Clearly, a private-coin protocol can be simulated by tossing the coins in public, so for every function $f:\left(\{0,1\}^{n}\right)^{k} \rightarrow\{0,1\}$, $R_{k}^{\text {pub }}(f) \leq R_{k}(f)$. In the other direction, [New91] provides a simulation of a publiccoin protocol by a private-coin protocol. Although it is stated for the special case of 2 players, the proof works for any number of players, in both NIH and NOF models.

Fact 2.5 ([New91]). There is a constant $c>0$ such that for every $k=k(n) \geq 2$, for every function family $f=\left(f_{k, n}\right)_{n}$, for every $\epsilon, \delta>0$,

$$
R_{k}^{\epsilon+\delta}\left(f_{k, n}\right) \leq R_{k}^{\mathrm{pub}, \epsilon}\left(f_{k, n}\right)+O\left(\log n+\log \frac{1}{\delta}\right)
$$

Public Coin Randomized vs. Distributional Using the min-max Theorem for zerosum games we get the following connection between the randomized and the distributional complexity of a function. This holds in both NIH and NOF models.

Fact 2.6 (Theorem 3.20 in [KN97]). For every function $f:\left(\{0,1\}^{n}\right)^{k} \rightarrow\{0,1\}$,

$$
R_{k}^{\mathrm{pub}}(f)=\max _{\text {distribution } \mu \text { on }\left(\{0,1\}^{n}\right)^{k}} D_{k}^{\mu}(f) .
$$

\subsubsection{The Discrepancy Method}

Let $f_{k, n}:\left(\{0,1\}^{n}\right)^{k} \rightarrow\{0,1\}$ be a boolean function, let $\mu$ be a distribution on $\left(\{0,1\}^{n}\right)^{k}$, and let $\epsilon<1 / 2$. Let $c$ be the cost of the best NIH deterministic protocol $P$ that computes $f$ with error at most $\epsilon$ under $\mu$. By Fact 2.2, $P$ induces a partition of $\left(\{0,1\}^{n}\right)^{k}$ into at most $2^{c} P$-monochromatic rectangles $R_{1}, \ldots, R_{2^{c}}$.

Consider a rectangle $R_{i}$ in this partition. If $P$ outputs $b \in\{0,1\}$ on $R_{i}$, then $R_{i}$ contributes $\mu\left(f^{-1}(1-b) \cap R_{i}\right)$ to the total error of $P$. Thus, it is better for $P$ to output 
the value $b$ for which $\mu\left(f^{-1}(b) \cap R_{i}\right)$ is larger. Still, since $P$ must output a single value on $R_{i}$, the contribution $\operatorname{err}\left(R_{i}\right)$ of $R_{i}$ to the total error in $P$ satisfies:

$$
\operatorname{err}\left(R_{i}\right) \geq \frac{\mu\left(R_{i}\right)-\operatorname{disc}_{\mu}\left(f, R_{i}\right)}{2} \geq \frac{\mu\left(R_{i}\right)-\operatorname{disc}_{\mathrm{NIH}, \mu}(f)}{2} .
$$

Summing over all rectangles $R_{i}$ we obtain the following lower bound on the distributional communication complexity of $f$. The statement holds in the NOF model as well.

Fact 2.7 (Proposition 3.28 in [KN97]). For every $\mathcal{M} \in\{\mathrm{NIH}, \mathrm{NOF}\}$, for every function $f_{k, n}:\left(\{0,1\}^{n}\right)^{k} \rightarrow\{0,1\}$, for every distribution $\mu$ on $\left(\{0,1\}^{n}\right)^{k}$, and for every $\epsilon<1 / 2$,

$$
D_{\mathcal{M}, k}^{\mu, \epsilon}\left(f_{k, n}\right) \geq \log \left(\frac{1-2 \cdot \epsilon}{\operatorname{disc}_{\mathcal{M}, \mu}\left(f_{k, n}\right)}\right) .
$$

Combining Facts 2.7 and 2.6 we see that by proving upper bounds for the discrepancy of a function under any distribution, we obtain lower bounds for its randomized communication complexity. This connection has been used in [CG88] to obtain randomized lower bounds for the Inner Product function (defined in Section 2.3) in the case of $k=2$ players.

If in the 2-player case the communication complexity of a function is ultimately defined in terms of "relatively benign" combinatorial objects (rectangles), this is no longer the case in the NOF model for $k>2$ players, where we have surprisingly few tools to handle cylinder intersections. Apart from non-constructive counting arguments and Ramsey-type results [CFL83, Pud03], which usually yield very weak bounds, essentially all known lower bounds use Facts 2.7 and 2.6. Still, it was unclear how to compute the NOF discrepancy of a function.

In a seminal paper, [BNS92] show that by $k$ repeated applications of the CauchySchwarz inequality $\mathrm{E}[X]^{2} \leq \mathrm{E}\left[X^{2}\right]$, we can reduce computing the NOF discrepancy of a function under the uniform distribution to computing the expected value of its product on the vertices of random $k$-dimensional cubes. Below, we write $\bar{x}$ for $\left(x_{1}, \ldots, x_{k}\right)$ and $\bar{x}^{u}$ for $\left(x_{1}^{u_{1}}, \ldots, x_{k}^{u_{k}}\right)$. 
Fact 2.8 ([BNS92]). For every function $f:\left(\{0,1\}^{n}\right)^{k} \rightarrow \mathbb{R}$ and for every cylinder intersection $T \in\left(\{0,1\}^{n}\right)^{k}$,

$$
\left(\underset{\bar{x} \sim \mathcal{U}\left(\left(\{0,1\}^{n}\right)^{k}\right)}{\mathrm{E}}\left[f(\bar{x}) \cdot 1_{T}(\bar{x})\right]\right)^{2^{k}} \leq \underset{\bar{x}^{0}, \bar{x}^{1} \sim \mathcal{U}\left(\left(\{0,1\}^{n}\right)^{k}\right)}{\mathrm{E}}\left[\prod_{u \in\{0,1\}^{k}} f\left(\bar{x}^{u}\right)\right] .
$$

In particular, if $f$ takes values in $\{-1,+1\}$ and $T$ is the cylinder intersection that witnesses the discrepancy of $f$ under the uniform distribution, then

$$
\left(\operatorname{disc}_{\mathrm{NOF}, \mathcal{U}}(f)\right)^{2^{k}} \leq \underset{\bar{x}^{0}, \bar{x}^{1} \sim \mathcal{U}\left(\left(\{0,1\}^{n}\right)^{k}\right)}{\mathrm{E}}\left[\prod_{u \in\{0,1\}^{k}} f\left(\bar{x}^{u}\right)\right] .
$$

This inequality has been rewritten in many ways [CT93, Raz00, FG05, VW08]. In this work, we use an alternative formulation where we stop just before the last application of the Cauchy-Schwarz inequality.

Fact 2.9 ([BNS92], [VW08]). For every function $f:\left(\{0,1\}^{n}\right)^{k} \rightarrow \mathbb{R}$ and for every cylinder intersection $T \subseteq\left(\{0,1\}^{n}\right)^{k}$,

$$
\left(\underset{\bar{x} \sim \mathcal{U}\left(\left(\{0,1\}^{n}\right)^{k}\right)}{\mathrm{E}}\left[f(\bar{x}) \cdot 1_{T}(\bar{x})\right]\right)^{2^{k-1}} \underset{\bar{y}^{0}, \bar{y}^{1} \sim \mathcal{U}\left(\left(\{0,1\}^{n}\right)^{k-1}\right)}{\mathrm{E}}\left[\mid \underset{x \sim \mathcal{U}\left(\{0,1\}^{n}\right)}{\mathrm{E}}\left[\prod_{u \in\{0,1\}^{k-1}} f\left(x, \bar{y}^{u}\right)\right]\right] .
$$

\subsection{Important Functions}

We denote by $\mathrm{OR}_{n}, \mathrm{AND}_{n}, \mathrm{XOR}_{n}$ the regular Or, And, and Xor functions on $n$ bits. We usually consider them over the range $\{0,1\}$, where we interpret 1 as true. Occasionally, we also consider them over the range $\{-1,+1\}$, where we interpret -1 as true.

\subsubsection{Equality}

For every $k=k(n)$, the Equality function family is $\mathrm{EQ}=\left(\mathrm{EQ}_{k, n}\right)_{n}$, where $\mathrm{EQ}_{k, n}$ : $\left(\{0,1\}^{n}\right)^{k} \rightarrow\{0,1\}$ is defined by $\mathrm{EQ}_{k, n}\left(x_{1}, \ldots, x_{k}\right):=1$ if and only if $x_{1}=\ldots=x_{k}$. The Inequality function family is INEQ $=\left(\mathrm{INEQ}_{k, n}\right)_{n}$, where $\mathrm{INEQ}_{k, n}:=1-\mathrm{EQ}_{k, n}$.

For the case of $k=2$ players, we know the following facts: 
- The deterministic communication complexity of both Equality and Inequality is linear: $D_{2}\left(\mathrm{EQ}_{2, n}\right) \geq \Omega(n)$ and $D_{2}\left(\mathrm{INEQ}_{2, n}\right) \geq \Omega(n)$.

- The nondeterministic communication complexity of the Equality function is also linear: $N_{2}\left(\mathrm{EQ}_{2, n}\right) \geq \Omega(n)$.

- Using a simple fingerprinting technique, the one-sided error randomized communication complexity of Inequality is $R_{2}^{1}\left(\mathrm{INEQ}_{2, n}\right) \leq O(\log n)$. As consequences, $R_{2}^{0}\left(\mathrm{EQ}_{2, n}\right) \leq O(\log n)$, and $N_{2}\left(\mathrm{INEQ}_{2, n}\right) \leq O(\log n)$.

In particular, Equality and Inequality witness that $\mathrm{P}_{2}^{\mathrm{cc}} \neq \mathrm{RP}_{2}^{\mathrm{cc}}$ and coRP $\mathrm{R}_{2}^{\mathrm{cc}} \nsubseteq \mathrm{NP}_{2}^{\mathrm{cc}}$.

Both functions are trivial in the NOF model when $k>2$, because if the inputs are not all equal, one of the players sees two different inputs without any communication.

\subsubsection{Set Intersection}

For every $k=k(n)$, the Set Intersection function family is SetInt $=\left(\operatorname{SetInt}_{k, n}\right)_{n}$, where SetInt $_{k, n}:\left(\{0,1\}^{n}\right)^{k} \rightarrow\{0,1\}$ is defined as follows. Let $x_{1}, \ldots, x_{k}$ be $n$-bit strings, and let $x_{i}=\left(x_{i, 1}, \ldots, x_{i, n}\right)$ be the individual bits. Then, $\operatorname{SetInt}_{k, n}\left(x_{1}, \ldots, x_{k}\right):=1$ if and only if there exists $j \in[n]$ such that for all $i \in[k], x_{i, j}=1$. Viewing the $x_{i}$ 's as the rows in a $k \times n$ matrix, the function is 1 if and only if there exists an all- 1 column. Alternatively, identifying each $n$-bit string $x_{i}$ with the characteristic vector of a subset $x_{i} \subseteq[n]$, we have SetInt $t_{k, n}=1$ if and only if the intersection of all $x_{i}{ }^{\prime}$ s is not empty: $\bigcap_{i=1}^{k} x_{i} \neq \varnothing$. The Set Disjointness function family is SetDisj $=\left(\text { SetDisj }_{k, n}\right)_{n}$, where $\operatorname{SetDisj}_{k, n}:=1-$ SetInt $_{k, n}$. The promise Set Intersection function family is pSetInt $=$ $\left(\text { pSetInt }_{k, n}\right)_{n}$, where pSetInt $k_{k, n}:=$ SetInt $_{k, n}$, except that the inputs satisfy the following promise:

Either the sets $x_{i}$ are mutually disjoint; or there exists a unique element $j$ in their intersection, and the sets $x_{i} \backslash\{j\}$ are mutually disjoint. 
Alternatively, in the matrix interpretation, the promise says there is at most one all-1 column, and all other columns contain at most a single 1. A protocol is free to output anything if given an input that does not satisfy the promise, hence computing the promise function is easier than the non-promise function, and lower bounds for the promise function imply lower bounds for the non-promise function.

For the case of $k=2$ player, we know the following facts:

- The deterministic communication complexities are all linear: $D_{2}\left(\right.$ SetInt $\left._{2, n}\right) \geq$ $D_{2}\left(\right.$ pSetInt $\left._{2, n}\right) \geq \Omega(n)$ and $D_{2}\left(\right.$ SetDisj $\left._{2, n}\right) \geq \Omega(n)$.

- The nondeterministic communication complexity of Set Disjointness is also linear: $N_{2}\left(\right.$ SetDisj $\left._{2, n}\right) \geq \Omega(n)$.

- In a nondeterministic protocol for Set Intersection, the players can simply guess an element in the intersection of the $x_{i}{ }^{\prime} \mathrm{s}$, so $N_{2}\left(\right.$ pSetInt $\left._{2, n}\right) \leq N_{2}\left(\right.$ SetInt $\left._{2, n}\right) \leq$ $O(\log n)$.

- [KS92, Raz90] show that the randomized communication complexity of promise Set Intersection (and hence, also of Set Disjointness) is linear: $R_{2}\left(\right.$ SetInt $\left._{2, n}\right) \geq$ $R_{2}\left(\right.$ pSetInt $\left._{2, n}\right) \geq \Omega(n)$ and $R_{2}\left(\right.$ SetDisj $\left._{2, n}\right) \geq \Omega(n)$.

In particular, Set Intersection witnesses that $\mathrm{NP}_{2}^{\mathrm{cc}} \nsubseteq \mathbb{\mathrm { BPP }}{ }_{2}^{\mathrm{cc}}$.

NIH It is easy to see that for Set Intersection and Set Disjointness, lower bounds from the case of $k=2$ players carry to the NIH model for more players. For example, a 2 player protocol $P$ can simulate a 3 player protocol $P^{\prime}$ as follows:

Let the players in $P$ be 1 and 2, and the players in $P^{\prime}$ be $A, B$, and $C$. In $P$, players 1 and 2 receive inputs $x_{1}$ and $x_{2}$, respectively. Henceforth, player 1 simulates the role of player $A$ with input $x_{1}$, and player 2 simulates the roles of both players $B$ and $C$, each with input $x_{2}$. 
This works because for Set Intersection and Set Disjointness, duplicating one of the sets $x_{i}$ does not change the output of the function.

However, the simple simulation above does not work for the promise Set Intersection function, because duplicating an input is easily seen to violate the promise in general. In fact, we know from [CKS03, Gro09] that $R_{\mathrm{NIH}, k}\left(\right.$ pSetInt $\left._{k, n}\right)=\Theta\left(\frac{n}{k}\right)$.

NOF It is easy to see that the promise Set Intersection function is trivial in the NOF model for $k \geq 3$ players. Recently, [LS08,CA08] showed that $R_{\mathrm{NOF}, k}\left(\operatorname{SetInt}_{k, n}\right) \geq n^{\Omega(1)}$ when $k<\log \log n$. Even more recently, this result was improved by [BHN09] to work for up to $k \leq \Theta\left((\log n)^{1 / 3}\right)$ players. The nondeterministic protocol from the 2-player case in fact works for SetInt ${ }_{k, n}$ for every $k$, so $N_{\mathrm{NOF}, k}\left(\operatorname{SetInt}_{k, n}\right) \leq O(\log n)$. In particular, these results imply that Set Intersection witnesses that $\mathrm{NP}_{k}^{\mathrm{cc}} \nsubseteq \mathrm{BPP}_{k}^{\mathrm{cc}}$ for $k \leq \Theta\left((\log n)^{1 / 3}\right)$.

\subsubsection{Inner Product}

For a prime power $q$, let $\mathbb{F}_{q}$ denote the finite field with $q$ elements. For every $k=k(n)$ and for every $q=q(n)$ which is a prime power, the Field Generalized Inner Product function family is FGIP $=\left(\operatorname{FGIP}_{k, n}^{\mathbb{F}_{q}}\right)_{n}$, where $\operatorname{FGIP}_{k, n}^{\mathbb{F}_{q}}:\left(\left(\mathbb{F}_{q}\right)^{n}\right)^{k} \rightarrow \mathbb{F}_{q}$ is defined as follows. For $i \in[k]$, let $x_{i}=\left(x_{i, 1}, \ldots, x_{i, n}\right) \in\left(\mathbb{F}_{q}\right)^{n}$, where for $j \in[n], x_{i, j} \in \mathbb{F}_{q}$. Then,

$$
\operatorname{FGIP}_{k, n}^{\mathbb{F}_{q}}\left(x_{1}, \ldots, x_{k}\right):=\sum_{j=1}^{n}\left(\prod_{i=1}^{k} x_{i, j}\right)
$$

where all operations are performed over $\mathbb{F}_{q}$. In the case when $q=2^{m}$, we identify $\mathbb{F}_{q}$ with $\{0,1\}^{m}$. So, in this case the domain of $\operatorname{FGIP}_{k, n}^{\mathbb{F}_{q}}$ is $\{0,1\}^{\mathrm{knm} m}$.

In the special case when $q=2$, we get the Generalized Inner Product function family GIP $=\left(\operatorname{GIP}_{k, n}\right)_{n}$ where $\operatorname{GIP}_{k, n}:=\mathrm{FGIP}_{k, n}^{\mathbb{F}_{2}}$. Furthermore, in the special case when $k=2$ and $q=2$, we get the usual Inner Product function family IP $=\left(\operatorname{IP}_{2, n}\right)_{n}$ where $\operatorname{IP}_{2, n}:=\mathrm{FGIP}_{2, n}^{\mathrm{F}_{2}}$. 
In the case of $k=2$ players, we know that Inner Product is hard for all types of protocols considered: $R_{2}\left(\mathrm{IP}_{2, n}\right) \geq \Omega(n)$ [CG88], $N_{2}\left(\mathrm{IP}_{2, n}\right) \geq \Omega(n)$, and $N_{2}^{0}\left(\mathrm{IP}_{2, n}\right) \geq$ $\Omega(n)$.

As in the case of the Set Intersection function, lower bounds from the 2-player case carry to the NIH model for more players.

Using the discrepancy method (see Section 2.2), [BNS92] proved the first strong lower bound in the NOF model for the communication complexity of the Generalized Inner Product function: $R_{\mathrm{NOF}, k}\left(\mathrm{GIP}_{k, n}\right) \geq \Omega\left(\frac{n}{4^{k}}\right)$. Observe that this bound becomes trivial $(\Omega(1))$ when $k \geq(1 / 2) \cdot \log n$. 


\section{Chapter 3}

\section{Deterministic vs. Randomized}

\section{Complexity}

In this chapter, we present the first major contribution of this work, which is a nonexplicit exponential separation between the powers of deterministic and one sided error randomized NOF communication protocols.

Theorem 3.1. For every function $k=k(N)$ satisfying $k \leq N^{O(1)}$, there exists a family of functions $F=\left(F_{k, N}\right)_{N}$, where $F_{k, N}:\left(\{0,1\}^{N}\right)^{k} \rightarrow\{0,1\}$, such that:

- $D_{\mathrm{NOF}, k}\left(F_{k, N}\right) \geq \Omega(N)$;

- $R_{\mathrm{NOF}, k}^{1, \mathrm{pub}}\left(F_{k, N}\right) \leq O(1) ;$ and

- $R_{\mathrm{NOF}, k}^{1}\left(F_{k, N}\right) \leq O(\log N)$.

In particular, the family $F$ witnesses the fact that $\mathrm{P}_{\mathrm{NOF}, k}^{\mathrm{cc}} \subsetneq \mathrm{RP}_{\mathrm{NOF}, k}^{\mathrm{cc}}$ for $k \leq N^{\mathrm{O}(1)}$.

In general, most lower bound proofs for deterministic NOF communication complexity use the fact shown in [BNS92] that any $k$-player protocol with complexity $d$ for a function $F$ yields a partitioning of the input into $O\left(2^{d}\right)$ disjoint cylinder intersections on which $F$ is constant. For $k \geq 3$ players, the known techniques for proving 
lower bounds on the number of cylinder intersections needed for such a partitioning are discrepancy-based and inherently yield lower bounds even for randomized protocols. Therefore, these techniques are not suitable for proving good lower bounds for functions with low randomized communication complexity.

The key idea that allows us to prove Theorem 3.1 is to consider a special class of boolean functions, called "graph" functions, which have the following two key properties. On the one hand, every graph function is reducible without communication to 2-player equality, which has a well known (fingerprint-based) efficient randomized protocol with bounded false-positives error. Thus, every graph function is in $\mathrm{coRP}_{k}^{\mathrm{cc}}$. On the other hand, we show that every deterministic protocol for a graph function can be put in a very special form, called an "oblivious-and-check" protocol, in which the bulk of the communication is done by a single player acting obliviously of all others, and the communication of every other player consists of a single check bit. The crux of the proof is that, by a counting argument, there are more graph functions than there are efficient oblivious-and-check protocols. Hence, some graph function has no efficient oblivious-and-check protocol, thus, no efficient deterministic protocol, placing it outside $\mathrm{P}_{k}^{c c}$.

In Section 3.1, we formally introduce graph functions and oblivious-and-check protocols. Then, we show that every graph function has an efficient randomized protocol. Next, we show that, in the case of graph functions, we can transform an efficient deterministic protocol into an efficient oblivious-and-check protocol. In Section 3.2, we give an alternative characterization of oblivious-and-check protocols, which ultimately allows us to count them. In Section 3.3, we give the actual counting argument that establishes Theorem 3.1.

As a corollary of Theorem 3.1, we also establish an optimal separation between the public-coin and private-coin randomized NOF models. We know from [KN97] that the largest possible gap between the public-coin randomized complexity of a function 
and its private-coin randomized complexity is achieved when the former is constant and the latter is logarithmic. We show that this gap can indeed be achieved.

Theorem 3.2. For every $\delta<1$ and for every function $k=k(N)$ satisfying $k<N^{\delta}$ for all sufficiently large $N$, there exists a family of functions $F=\left(F_{k, N}\right)_{N}$, where $F_{k, N}:\left(\{0,1\}^{N}\right)^{k} \rightarrow$ $\{0,1\}$, such that:

- $R_{\mathrm{NOF}, k}\left(F_{k, N}\right)=\Theta(\log N)$; and

- $R_{\mathrm{NOF}, k}^{\mathrm{pub}}\left(F_{k, N}\right) \leq O(1)$.

We prove Theorem 3.2 in Section 3.4.

Finally, in Section 3.5, we consider the problem of making the above separations explicit. We introduce a certain "mixing" property, we prove a (weak) lower bound of $\Omega(\log N)$ for the deterministic communication complexity of any graph function satisfying this mixing property, and we provide some evidence supporting the conjecture that, in fact, the lower bound is super-polylogarithmic.

The work in this chapter appears in [BDPW07]. 1 Throughout, the number of players in a protocol is given by the function $k=k(N)$, and we exclusively discuss the NOF model of communication complexity.

\subsection{Special Functions and Protocols}

The functions we use in the proof of Theorem 3.1 satisfy the following property.

Definition 3.3. We say that $F:\left(\{0,1\}^{N}\right)^{k} \rightarrow\{0,1\}$ is a graph function if for all $\left(x_{2}, \ldots, x_{k}\right)$, there exists at most one $x_{1}^{*}$ such that $F\left(x_{1}, x_{2}, \ldots, x_{k}\right)=1$.

First, we show that every graph function has an efficient randomized protocol.

\footnotetext{
${ }^{1}$ The function used in Section 3.5.2 is different. In [BDPW07], we used a construction based on [BV02, Vee04]. The construction given in this work is cleaner, as it simply adapts a result from [BHK01].
} 
Lemma 3.4. Let $F=\left(F_{k, N}\right)_{N}$ be a family of functions such that $F_{k, N}:\left(\{0,1\}^{N}\right)^{k} \rightarrow\{0,1\}$ is graph for all sufficiently large $N$. Then, $R_{k, 1 / n}^{0}\left(F_{k, N}\right) \leq O(\log n)$ and $R_{k}^{0, p u b}\left(F_{k, N}\right) \leq$ $O(1)$. In particular, $F \in \operatorname{coRP}_{k}^{c c}$.

Proof of Lemma 3.4. Observe that if $F_{k, N}$ is graph, then it is reducible with no communication to 2-player $N$-bit equality $\mathrm{EQ}_{2, N}$ : player 1 , seeing $\left(x_{2}, \ldots, x_{k}\right)$, computes the unique value $x_{1}^{*}$ for which $F_{k, N}\left(x_{1}, x_{2}, \ldots, x_{k}\right)=1$ (if it exists); any other player $j>1$ sees the real input $x_{1}$; now players 1 and 2 run the 2-player randomized protocol testing whether $x_{1}^{*}=x_{1}$. We know that $R_{2}^{0,1 / N}\left(\mathrm{EQ}_{2, N}\right) \leq O(\log N)$ and $R_{2}^{0, p u b}\left(\mathrm{EQ}_{2, N}\right) \leq$ $O(1)$ [KN97].

Next, we show that, for a graph function, we can efficiently convert any deterministic protocol to the following special type of protocol.

Definition 3.5. Let $P$ be a deterministic protocol. We say that $P$ is an oblivious-andcheck protocol if on every input $x=\left(x_{1}, x_{2}, \ldots, x_{k}\right)$, player 1 first communicates a message, possibly consisting of many bits (the term "oblivious" reflects the fact that this message is not function of any other communication); then every player $j>1$ simultaneously communicates a single bit $b_{j}$, based on the input player $j$ sees and on the initial message from player 1 ; and now the output of $P$ is 1 if and only if $b_{j}=1$ for all $j>1$ (this is reflected in the term "check").

Lemma 3.6. Let $F:\left(\{0,1\}^{N}\right)^{k} \rightarrow\{0,1\}$ be a graph function and let $P$ be a deterministic protocol for $F$ with cost $c$. Then, there exists an oblivious-and-check protocol $P^{\prime}$ for $F$ in which the initial message consists of $c$ bits.

Proof of Lemma 3.6. We describe the protocol $P^{\prime}$ on input $\left(x_{1}, x_{2}, \ldots, x_{k}\right)$.

Player 1 (call her Alice) sees the partial input $\left(x_{2}, \ldots, x_{k}\right)$. She privately computes $x_{1}^{*} \in\{0,1\}^{N}$, the value for which $F\left(x_{1}^{*}, x_{2}, \ldots, x_{k}\right)=1$, if it exists. If such a value does not exist, she lets $x_{1}^{*}$ be arbitrary. 
Next, Alice "simulates" the protocol $P$ on input $\left(x_{1}^{*}, x_{2}, \ldots, x_{k}\right)$. That is, she writes on the blackboard the string $\tau$ that would have been written collaboratively by players $1, \ldots, k$ if $P$ were executed on $\left(x_{1}^{*}, x_{2}, \ldots, x_{k}\right)$.

Then, each player $i>1$ verifies that $\tau$ is consistent with what that player would have sent in protocol $P$ on input $\left(x_{1}, x_{2}, \ldots, x_{k}\right)$. More precisely, player $i$ (call him Bob) reads the bits $\tau_{1}, \tau_{2}, \ldots$ of $\tau$, until he has read a partial message $\tau_{1}, \ldots, \tau_{j}$ upon which, in protocol $P$, it would be Bob's turn to write the next bit. Now, Bob checks whether the bit he would write in $P$ (on input $\left(x_{1}, x_{2}, \ldots, x_{k}\right)$ ) does in fact coincide with $\tau_{j+1}$ (which is what Alice computed Bob would write on input $\left(x_{1}^{*}, x_{2}, \ldots, x_{k}\right)$ ). This is repeated until Bob has read all bits from $\tau$. In addition, Bob also checks that the output of $P$ is 1 when the blackboard content is $\tau$. If Bob does not find an error, and $P$ outputs 1 on $\tau$, then he sends bit $b_{i}=1$. Otherwise he sends $b_{i}=0$.

Consider an input $\left(x_{1}, x_{2}, \ldots, x_{k}\right)$, and let $\tau$ be the message sent by Alice for that input. If there is no $x_{1}^{*}$ such that $F\left(x_{1}^{*}, x_{2}, \ldots, x_{k}\right)=1$, no matter what $x_{1}^{*}$ is chosen by Alice (even when $x_{1}^{*}=x_{1}$ ), the blackboard contents $\tau$ imply an output of 0 in protocol $P$. Hence, every player $i>1$ communicates $b_{i}=0$, so $P^{\prime}$ outputs 0 , which is correct because $F\left(x_{1}, x_{2}, \ldots, x_{k}\right)=0$ in this case.

Now assume that there is a unique $x_{1}^{*}$ such that $F\left(x_{1}^{*}, x_{2}, \ldots, x_{k}\right)=1$, and hence $\tau$ is the blackboard contents for protocol $P$ on input $\left(x_{1}^{*}, x_{2}, \ldots, x_{k}\right)$.

If $x_{1}=x_{1}^{*}$, clearly every player $i>1$ communicates $b_{i}=1$. Hence, $P^{\prime}$ correctly computes outputs 1 .

Finally, assume that $x_{1} \neq x_{1}^{*}$, and hence $F\left(x_{1}, x_{2}, \ldots, x_{k}\right)=0$. Let $\tau^{\prime}$ be the blackboard contents in protocol $P$ on input $\left(x_{1}, x_{2}, \ldots, x_{k}\right)$. Since $F\left(x_{1}, x_{2}, \ldots, x_{k}\right) \neq$ $F\left(x_{1}^{*}, x_{2}, \ldots, x_{k}\right)$, we have $\tau^{\prime} \neq \tau$. Let $j$ be the index of the first bit different in $\tau^{\prime}$ from $\tau$. Note that, at any time before the $j$-th bit is written on the blackboard, the information Alice obtains from the blackboard and the other players' foreheads is the same for the inputs $\left(x_{1}, x_{2}, \ldots, x_{k}\right)$ and $\left(x_{1}^{*}, x_{2}, \ldots, x_{k}\right)$. Therefore, Alice does not write the 
$j$-th bit in protocol $P$. Hence, there must be some other player $i>1$ whose messages in $P$ on input $\left(x_{1}, x_{2}, \ldots, x_{k}\right)$ differ from $\tau$. This player sends $b_{i}=0$ in the protocol $P^{\prime}$, and thus $P^{\prime}$ correctly outputs 0 in this case.

\subsection{An Alternative Characterization}

Some of the functions we study are defined as embeddings of other functions in the domain $\left(\{0,1\}^{N}\right)^{k}$, and, as a result, they are constant (usually, 0 ) unless their inputs a appropriately 0-padded. For such functions, we use the term effective domain to refer to the portion of the input bits that really influence the output.

Definition 3.7. For a function $F:\left(\{0,1\}^{N}\right)^{k} \rightarrow\{0,1\}$, the effective domain for player $i$ is the set $X_{i} \subseteq\{0,1\}^{N}$ defined by

$$
x_{i} \in X_{i} \Longleftrightarrow \exists\left(x_{1}, \ldots, x_{i-1}, x_{i+1}, \ldots, x_{k}\right) \text { such that } F\left(x_{1}, \ldots, x_{k}\right)=1 .
$$

We say that $X_{1} \times \ldots \times X_{k}$ is the effective domain of $F$ when for every $i, X_{i}$ is the effective domain for player $i$.

For a natural number $D$ and a set $S$, a $D$-coloring of $S$ is a mapping $\chi: S \rightarrow[D]$. The following is an alternative representation of functions computed by obliviousand-check protocols.

Lemma 3.8. Let $F:\left(\{0,1\}^{N}\right)^{k} \rightarrow\{0,1\}$ be a function that is computed by an obliviousand-check protocol $P^{\prime}$ in which the first message consists of $c$ bits. Let $X_{1}$ be the effective domain of $F$ for player 1 . Then, there exists a set of $(k-1)$-dimensional cylinder intersections $\mathcal{I}=\left\{I_{m, a} \subseteq\left(\{0,1\}^{N}\right)^{k-1} \mid m \in\left[2^{c}\right], a \in X_{1}\right\}$, and a $2^{c}$-coloring $\chi$ of $\left(\{0,1\}^{N}\right)^{k-1}$, such that

$$
\forall x=\left(x_{1}, x_{2}, \ldots, x_{k}\right) \in\left(\{0,1\}^{N}\right)^{k}, \quad F(x)=1 \Leftrightarrow\left(x_{2}, \ldots, x_{k}\right) \in I_{\chi\left(x_{2}, \ldots, x_{k}\right), x_{1}} .
$$

In particular, $I_{m, a}$ contains all points $y \in\left(\{0,1\}^{N}\right)^{k-1}$ with color $\chi(y)=m$ and $F(a, y)=1$, but no point $y^{\prime}$ with color $\chi\left(y^{\prime}\right)=m$ and $F\left(a, y^{\prime}\right)=0$. 
Proof of Lemma 3.8. First, by definition of the effective domain, $F\left(a^{\prime}, x_{2}, \ldots, x_{k}\right)=0$ for every $a^{\prime} \notin X_{1}$ and for every $\left(x_{2}, \ldots, x_{k}\right)$.

Let $\chi$ be the function according to which Alice speaks in $P^{\prime}$, so $\chi\left(x_{2}, \ldots, x_{k}\right) \in\left[2^{c}\right]$ is the $c$-bit message Alice sends in $P^{\prime}$ if she sees $\left(x_{2}, \ldots, x_{k}\right)$. Let $m \in\left[2^{c}\right]$ be a fixed message from Alice, let $a \in X_{1}$ be a fixed value on Alice's forehead, and consider player $i>1$ (Bob). The set of points $\left(x_{2}, \ldots, x_{k}\right) \in\left(\{0,1\}^{N}\right)^{k-1}$ for which Bob communicates $b_{i}=1$ when he sees $a$ on Alice's forehead and gets initial message $m$ from Alice is a cylinder in the $(i-1)$-st dimension (note, in $\left(\{0,1\}^{N}\right)^{k-1}$, dimension 1 corresponds to player 2). Hence, the set of points $\left(x_{2}, \ldots, x_{k}\right) \in\left(\{0,1\}^{N}\right)^{k-1}$ for which all players $i>1$ communicate $b_{i}=1$ is a $(k-1)$-dimensional cylinder intersection $I_{m, a}$.

To prove the if and only if, first consider an $x$ such that $F(x)=1$. On this input, Alice communicates $\chi\left(x_{2}, \ldots, x_{k}\right)$, and all players $i>1$ communicate $b_{i}=1$. Hence, by definition, $\left(x_{2}, \ldots, x_{k}\right) \in I_{\chi\left(x_{2}, \ldots, x_{k}\right), x_{1}}$.

Finally, consider an $x$ such that $\left(x_{2}, \ldots, x_{k}\right) \in I_{\chi\left(x_{2}, \ldots, x_{k}\right), x_{1}}$. In $P^{\prime}$, the first message of Alice is by definition $\chi\left(x_{2}, \ldots, x_{k}\right)$. Subsequently, all players $i>1$ send $b_{i}=1$ because they see $x_{1}$ on Alice's forehead and they get $\chi\left(x_{2}, \ldots, x_{k}\right)$. Hence, $F(x)=1$.

In the representation from Lemma 3.8, each cylinder intersection $I_{m, a}$ may also contain points that are not colored $m$. However, it is not possible that a point $\left(x_{2}, \ldots, x_{k}\right) \in$ $I_{m, a}$ has color $m$ but $P^{\prime}\left(a, x_{2}, \ldots, x_{k}\right)=0$ because then Alice would send message $m$ if she saw $\left(x_{2}, \ldots, x_{k}\right)$ and the other players would all accept if they saw $a$ on Alice's forehead. Hence, $\left(a, x_{2}, \ldots, x_{k}\right)$ would be accepted by $P^{\prime}$, a contradiction.

Furthermore, the pair $(\mathcal{I}, \chi)$ in Lemma 3.8 is not unique. First, $F$ can be computed by several different protocols, which results in different representations. Second, even when considering a single protocol $P^{\prime}$ for $F$, if Alice never sends a particular message $m$ in $P^{\prime}$, then, the choice of cylinder intersections $I_{m, a}$ for $a \in X_{1}$ is arbitrary in the representation on $F$. To be able to talk about "the" alternative representation of $F$, we 
break ties arbitrarily.

Definition 3.9. For a given $c$, let $F$ be a function that can be computed by some oblivious-and-check protocol $P^{\prime}$ in which the first message consists of $c$ bits. The alternative representation of $F$ is the lexicographically smallest (over an arbitrary alphabet) pair $(\mathcal{I}, \chi)$ satisfying the condition in Lemma 3.8.

An important property of the alternative representation is that it can be used to reconstruct the function value on any given point. Thus, we get the following immediate result.

Corollary 3.10. For a given $c$, let $F_{1}, F_{2}$ be different functions. Let $P_{1}^{\prime}, P_{2}^{\prime}$ be oblivious-andcheck protocols in which the first message consists of $c$ bits, computing $F_{1}, F_{2}$, respectively, Then, the alternative representations of $F_{1}$ and $F_{2}$ are different.

\subsection{A Lower Bound for Deterministic Protocols}

Below, we consider a class of functions that have logarithmic communication complexity for private-coin randomized protocols with bounded one-sided error. Using Lemma 3.8 we give an upper bound on the number of efficient deterministic protocols for the functions in that class in order to show that at least one such function requires at least linear deterministic communication complexity.

For every function $m=m(N)$ satisfying $m \leq N$, let $G_{k-1, N, m}$ be the set of all mappings $g:\left(\{0,1\}^{N}\right)^{k-1} \rightarrow\{0,1\}^{m}$. For every $g \in G_{k-1, N, m}$, define $F_{k, N}^{g}:\left(\{0,1\}^{N}\right)^{k} \rightarrow$ $\{0,1\}$ by $F_{k, N}^{g}\left(x_{1}, \ldots, x_{k}\right)=1$ if and only if $x_{1}=g\left(x_{2}, \ldots, x_{k}\right) \circ 0^{N-m}$. That is, the first $m$ bits of $x_{1}$ are $g\left(x_{2}, \ldots, x_{k}\right)$, and the last $N-m$ bits are 0 . Note that the effective domain of $F_{k, N}^{g}$ is $\{0,1\}^{m} \times\left(\{0,1\}^{N}\right)^{k-1}$. The following Lemma is the heart of our counting argument. 
Lemma 3.11. There exist a function $m=m(N) \leq N$ such that for all sufficiently large $N$, there exists a function $g \in G_{k-1, N, m}$ such that $D_{k}\left(F_{k, N}^{g}\right) \geq \Omega(N-\log k)$. In particular, for the function family $F=\left(F_{k, N}^{g}\right)_{N}$, we have $F \notin \mathrm{P}_{k}^{c c}$.

Assuming we have proved Lemma 3.11 above, we complete the proof of the main Theorem in this chapter.

Proof of Theorem 3.1. Let $m$ and $g$ be the functions given by Lemma 3.11, achieving the deterministic lower bound. The randomized upper bounds follow from Lemma 3.4, since for every $g \in G_{k-1, N, m}, F_{k, N}^{g}$ is graph.

We now give the proof our central counting argument.

Proof of Lemma 3.11. A function $g \in G_{k-1, N, m}$ has a domain of size $2^{(k-1) N}$ and a range of size $2^{m}$. Therefore, it is not possible to encode every such $g$ with less than $m \cdot 2^{(k-1) N}$ bits. Note that if two functions $g, g^{\prime}$ are different, then $F_{k, N}^{g}$ and $F_{k, N}^{g^{\prime}}$ are different, too. We write $F^{g}$ for $F_{k, N}^{g}$.

Clearly, for all $g \in G_{k-1, N, m}, F^{g}$ is graph. Let $d=d(N)$ be a value such that for all $g \in G_{k-1, N, m}, F^{g}$ has deterministic complexity $D_{k}\left(F^{g}\right) \leq d$. Let $P^{g}$ be a deterministic protocol for $F^{g}$ of cost $d$. Let $P^{\prime g}$ be the oblivious-and-check protocol for $F^{g}$ given by Lemma 3.6, in which the first message consists of $d$ bits.

Since the effective domain of $F_{g}$ for player 1 is $(\{0,1\})^{m}$, by Lemma $3.8, F^{g}$ can be represented by a $2^{d}$-coloring of $\left(\{0,1\}^{N}\right)^{k-1}$ and $2^{m} \cdot 2^{d}$ cylinder intersections in $\left(\{0,1\}^{N}\right)^{k-1}$. The $2^{d}$-coloring can be encoded on $d \cdot 2^{(k-1) N}$ bits. The number of $i$ cylinders in $X_{1} \times \ldots \times X_{t}$ is $2^{\prod_{j \neq i}\left|X_{j}\right|}$. Hence, $(k-1) \cdot 2^{(k-2) N}$ bits suffice to encode a cylinder intersection in $\left(\{0,1\}^{N}\right)^{k-1}$. Thus, the alternative representation of $F^{g}$ can be encoded on at most

$$
d \cdot 2^{(k-1) N}+2^{d+m} \cdot(k-1) \cdot 2^{(k-2) N}=d \cdot 2^{(k-1) N}+(k-1) \cdot 2^{d+m+(k-2) N}
$$


By Corollary $3.10, F^{g}$ and $F^{g^{\prime}}$ must have different alternative representations when $g \neq g^{\prime}$. Since the number of bits needed to describe a function $F^{g}$ for $g \in G_{k-1, N, m}$ is at least $m \cdot 2^{(k-1) N}$, we get:

$$
d \cdot 2^{(k-1) N}+(k-1) \cdot 2^{d+m+(k-2) N} \geq m \cdot 2^{(k-1) N} .
$$

This is equivalent to $2^{d} \geq 2^{N-m} \cdot(m-d) /(k-1)=2^{N-m-\log (k-1)} \cdot(m-d)$. Hence, either $d \geq m$ or $d \geq N-m-\log (k-1)$. Setting $m=\lfloor(N-\log (k-1)) / 2\rfloor$, we get $d \geq(N-\log k) / 2-O(1)$.

\subsection{Public vs. Private Coins}

We now consider the difference between public-coin and private-coin randomized protocols. Trivially, any private-coin protocol can be simulated by tossing the coins in public, so for all $F$ and $k, R_{k}^{\mathrm{pub}}(F) \leq R_{k}(F)$. By Fact 2.5 ([New91]), $R_{k}(F) \leq$ $O(1) \cdot R_{k}^{\text {pub }}(F)+O(\log N)$. We see that the maximum gap between the public-coin and private-coin randomized complexities of $F$ is $\Theta(\log N)$, and it is achieved when $R_{k}^{\text {pub }}(F)$ is $O(1)$ and $R_{k}(F)$ is $\Theta(\log N)$. The natural question arises, is there a function that achieves this gap? Our results allow us to answer this question affirmatively.

To obtain lower bounds, we need the following extension of Fact 2.4 (Lemma 3.8 in [KN97]) to $k$ players.

Lemma 3.12. For every constant $0<\delta<1$, for every function $k=k(N)$, and for every function family $F=\left(F_{k, N}\right)_{N}$, where $F_{k, N}:\left(\{0,1\}^{N}\right)^{k} \rightarrow\{0,1\}$, the following holds. If $D_{k}\left(F_{k, N}\right)$ is increasing and $k<D_{k}\left(F_{k, N}\right)^{\delta}$ for all sufficiently large $N$, then $R_{k}(F) \geq \Omega\left(\log D_{k}(F)\right)$.

Proof of Lemma 3.12. We write $F$ for $F_{k, N}$. First, we claim the following holds for every $\epsilon<1 / 2$ and for all sufficiently large $N$ :

$$
D_{k}(F) \leq(k-1) \cdot 2^{R_{k}^{\epsilon}(F)} \cdot\left(1+\log (k-1)-\log \left(\frac{1}{2}-\epsilon\right)+R_{k}^{\epsilon}(F)\right)
$$


Provided we prove the above statement, assume that $R_{k}(F) \leq \frac{1-\delta}{2} \cdot \log D_{k}(F)$. Recall that $R_{k}(F)=R_{k}^{1 / 3}(F)$. Then,

$$
\begin{aligned}
D_{k}(F) & =O\left(D_{k}(F)^{\delta} \cdot D_{k}(F)^{\frac{1-\delta}{2}} \cdot\left(\delta \cdot \log D_{k}(F)+\frac{1-\delta}{2} \cdot \log D_{k}(F)\right)\right) \\
& =O\left(D_{k}(F)^{\frac{1+\delta}{2}} \cdot \log D_{k}(F)\right)
\end{aligned}
$$

Since $\delta<1$ and $D_{k}(F)$ is increasing, this cannot hold for all $N$, which is a contradiction. Hence, $R_{k}(F)=\Omega\left(\log D_{k}(F)\right)$.

The proof of our initial claim follows the same idea as the proof of Lemma 3.8 in [KN97]. Let $c=R_{k, \epsilon}(F)$ and consider an $\epsilon$-error randomized protocol $P$ of cost $c$. For a $t$ that we determine later, we construct a deterministic protocol $P^{\prime}$ for $F$ of cost $(k-1) \cdot 2^{c} \cdot t$ as follows.

For every player $i$ and every leaf $l$ of the randomized protocol $P$, let $p_{i}(l)$ denote the probability player $i$ responds according to the path leading to $l$ (over their own private random strings). Player $i$ (for $1 \leq i \leq k-1$ ) computes, for every leaf $l$, the real number $p_{i}(l)$ and publishes $p_{i}^{*}(l)$, a $t$-bit approximation of $p_{i}(l)$. This introduces an error of at most $\phi=2^{-t}$ for every such value.

Player $k$ now computes, for every $l$, the value $\left(\prod_{i=1}^{k-1} p_{i}^{*}(l)\right) \cdot p_{k}(l)$. Since $p_{i}^{*}(l) \in$ $\left\{p_{i}(l)-\phi, p_{i}(l)+\phi\right\}$ and since $p_{i}(l) \leq 1$, we get

$$
\left(\prod_{i=1}^{k-1} p_{i}^{*}(l)\right) \cdot p_{k}(l) \in\left\{\prod_{i=1}^{k} p_{i}(l)-\left((1+\phi)^{k-1}-1\right), \prod_{i=1}^{k} p_{i}(l)+\left((1+\phi)^{k-1}-1\right)\right\} .
$$

Each leaf of the randomized protocol is associated with an output (0 or 1$)$. Player $k$ now estimates the probability of an output in the randomized protocol by summing over the estimates of the probabilities for each leaf corresponding to that output. Finally, player $k$ decides to output in $P^{\prime}$ the value that has a probability higher than $1 / 2$.

In the original protocol $P$, an error is made with probability at most $\epsilon$. For the deterministic simulation to work, we need to make sure that the extra error introduced 
by the rounding process is less than $1 / 2-\epsilon$. Since each estimate has error at most $(1+\phi)^{k-1}-1$ and there are at most $2^{c}$ leaves, we need to make sure that $2^{c} \cdot((1+$ $\left.\phi)^{k-1}-1\right)<(1 / 2-\epsilon)$. Equivalently, we need $(1+\phi)^{k-1}<1+(1 / 2-\epsilon) / 2^{c}$.

Let $t=1+\log (k-1)-\log (1 / 2-\epsilon)+c$. Then, $\phi=2^{-t}=\frac{1 / 2-\epsilon}{2 \cdot(k-1) 2^{2}}$. We know that, for $0 \leq x<1 / 2$, we have $(1+x /(k-1))^{k-1} \leq e^{x} \leq 1+2 \cdot x$. Moreover, $(1 / 2-\epsilon) /\left(2 \cdot 2^{c}\right)<1 / 2$. Therefore,

$$
(1+\phi)^{k-1}<1+2 \cdot \frac{1 / 2-\epsilon}{2 \cdot 2^{c}}=1+\frac{1 / 2-\epsilon}{2^{c}} .
$$

Thus, for this setting of parameters, $P^{\prime}$ is correct. This completes the proof of the claim, hence also the proof of the Lemma.

We can now complete the proof of our Theorem giving the optimal separation between public-coin and private-coin randomized NOF protocols.

Proof of Theorem 3.2. Fix a constant $0<\delta<1$ and a function $k=k(N)$ satisfying $k<$ $N^{\delta}$ for all sufficiently large $N$. By Theorem 3.1 there is a function family $F=\left(F_{k, N}\right)_{N}$ such that $D_{k}\left(F_{k, N}\right) \geq \Omega(N), R_{k}^{\mathrm{pub}}\left(F_{k, N}\right) \leq O(1)$, and $R_{k}\left(F_{k, N}\right) \leq O(\log N)$.

Let $\delta^{\prime}:=(1+\delta) / 2$. Then, $0<\delta<\delta^{\prime}<1$ and $k<N^{\delta}<D_{k}\left(F_{k, N}\right)^{\delta^{\prime}}$ for all sufficiently large $N$. By Lemma $3.12, R_{k}\left(F_{k, N}\right) \geq \Omega(\log N)$. Thus, $R_{k}\left(F_{k, N}\right)=$ $\Theta(\log N)$.

\subsection{Explicit Functions}

The result given in Theorem 3.1 is non-constructive, we simply show that there exists a function family separating deterministic and randomized protocols. We conjecture that there also exist explicit graph functions that give a linear or near linear separation between deterministic and one-sided error randomized $k$-player NOF communication complexities. In this section, we give two constructions of explicit graph functions (which are in $\operatorname{coRP}_{k}^{c c}$ by Lemma 3.4) that, we believe, might be outside $\mathrm{P}_{k}^{c c}$ for $k \geq 3$. 
While we are unable to prove the super-polylogarithmic $\left((\log N)^{\omega(1)}\right)$ deterministic communication complexity lower bounds required to place them outside $\mathrm{P}_{k}^{c c}$, we are, however, able to prove much weaker logarithmic $(\Omega(\log N))$ lower bounds. As a corollary, we obtain a separation between deterministic and public coin randomized communication complexities of graph functions, though this is much weaker than our conjecture.

We begin in Section 3.5.1, by giving a family of graph functions for the case where we have only $k=3$ players. This construction does not immediately generalize for $k>3$ players, but we present it first because it is both simple enough and illustrative of a certain "mixing" property (made precise later) that plays a crucial role in our arguments. We then prove the lower bound $D_{k}(F) \geq \Omega(\log N)$ for, in fact, any function $F$ that satisfies this mixing property. In Section 3.5.2, we present a different family of graph functions, that can be defined for any $k \geq 3$, and we show that for $3 \leq k=k(N) \leq(1 / 9) \cdot \log N$, this function satisfies the same mixing property that we use in Section 3.5.1, thus allowing us to obtain a similar lower bound.

\subsubsection{A Function Family for Three Players}

In the case of $k=3$ players, our construction is based on universal families of hash functions. We begin with an informal description of our arguments.

Given a universal family $H$ of hash functions from $A$ to $B$, our three player function $F$ is defined on the set $B \times H \times A$ as follows. The second player holds a hash function $h \in H$, the third player holds an input $x \in A$, and $h(x) \in B$ is the unique value for the input of the first player that makes $F$ evaluate to 1 . The key "mixing" property that this construction satisfies is closely related to the Hash Mixing Lemma from [MNT93], and says that, for every large rectangle $R \subseteq H \times A$ and every $b \in B$, if we visualize $R$ as a matrix where the entry at location $(h, x)$ has value $h(x)$, then the number of $b$-valued entries in $R$ is very close to uniform, that is, $|R| /|B|$. After making these 
definitions precise, we use the mixing property combined with the alternative characterization of graph functions from Lemma 3.8, to give some evidence as to why we believe $D_{k}(F)$ might be large. Then, we prove in Theorem 3.17 that $D_{k}(F) \geq \Omega(\log n)$ for any function $F$ that satisfies the mixing property.

Definition of the Family. We write $\mathbb{F}_{q}$ for the finite field of $q$ elements when $q$ is a prime power. Let $n \geq 4$ be a positive integer, and let $m:=n^{1 / 2}$. For $x \in\{0,1\}^{N}$ and $a \in\{0,1\}^{n+m-1}$, let $a \odot x$ (the convolution of $a$ and $x$ ) be the $m$-bit string $z$ whose $i$-th bit is defined by $z_{i}=\sum_{j=1}^{n} x_{j} a_{(i-1)+j} \bmod 2$. For two $m$-bit strings $z$ and $b$, let $z \oplus b$ be the bitwise exclusive-or of the two strings. For $(a, b) \in \mathbb{F}_{2^{n+m-1}} \times \mathbb{F}_{2^{n}}$, let $h_{a, b}: \mathbb{F}_{2^{n}} \rightarrow \mathbb{F}_{2^{m}}$ be defined by $h_{a, b}(x)=(a \odot x) \oplus b$. [MNT93] show that $\mathcal{H}^{n, m}=$ $\left\{h_{a, b} \mid a \in \mathbb{F}_{2^{n+m-1}}, b \in \mathbb{F}_{2^{n}}\right\}$ is a universal family of hash functions from $\mathbb{F}_{2^{n}}$ to $\mathbb{F}_{2^{m}}$. We are now ready to define our family of graph functions for $k=3$ players.

Definition 3.13. Let $F=\left(F_{3, N}\right)_{N}$ be the family where $F_{3, N}:\left(\{0,1\}^{N}\right)^{3} \rightarrow\{0,1\}$ is defined as follows. Let $n:=N / 3$ and let $m:=n^{1 / 2}$. On input $\left(x_{1}, x_{2}, x_{3}\right), F_{3, N}$ is 0 unless:

(i) $x_{1}=y \circ 0^{N-m}$,

(ii) $x_{2}=a \circ b \circ 0^{N-2 n-m+1}$,

(iii) $x_{3}=x^{\prime} \circ 0^{N-n}$, and

(iv) $h_{a, b}\left(x^{\prime}\right)=y$.

In other words, $F_{3, N}$ is 0 unless its arguments are 0 -padded encodings of $y \in \mathbb{F}_{2^{m}}$, $h \in \mathcal{H}^{n, m}$ and $x^{\prime} \in \mathbb{F}_{2^{n}}$, such that $h\left(x^{\prime}\right)=y$. Note that the effective domain of $F_{3, N}$ is $\mathbb{F}_{2^{m}} \times \mathcal{H}^{n, m} \times \mathbb{F}_{2^{n}}$.

Clearly, $F_{3, N}$ is a graph function, so by Lemma $3.4, R_{3}^{0,1 / N}\left(F_{3, N}\right)=O(\log N)$ and $R_{3}^{0, \text { pub }}\left(F_{3, N}\right)=O(1)$. 
Mixing Property. The following is a "mixing" property of $F$, that we will be use to prove lower bounds on $D_{3}(F)$.

Definition 3.14 (Mixing Property). Let $F=\left(F_{k, N}\right)$ be a function family where $F_{k, N}$ : $\left(\{0,1\}^{N}\right)^{k} \rightarrow\{0,1\}$ is a graph function with effective domain $X_{1} \times \ldots \times X_{k}$. Let $Z=X_{2} \times \ldots \times X_{k}$ and let $m=\log \left|X_{1}\right|$. Let $g: Z \rightarrow X_{1} \cup\{\perp\}$ be the function defined by $g\left(x_{2}, \ldots, x_{k}\right):=x_{1}$ if there exists an $x_{1}$ such that $F_{k, N}\left(x_{1}, x_{2}, \ldots, x_{k}\right)=1$, and $g\left(x_{2}, \ldots, x_{k}\right):=\perp$ otherwise. We say that the function family $F$ has the mixing property if the following holds. For large enough $N$, for every cylinder intersection $I \subseteq Z$ with $|I| \geq|Z| \cdot 2^{-2 m}$, and for every $x_{1} \in X_{1}$, when $\left(x_{2}, \ldots, x_{k}\right)$ is drawn from $\mathcal{U}(I)$, the uniform distribution on $I$,

$$
\underset{\left(x_{2}, \ldots, x_{k}\right) \sim \mathcal{U}(I)}{\operatorname{Pr}}\left[g\left(x_{2}, \ldots, x_{k}\right)=x_{1}\right] \leq 2 \cdot \frac{1}{2^{m}}=2^{-m+1} .
$$

The intuition behind this definition is that, if $g$ were a true random function, $22 \mathrm{we} \mathrm{e}^{\prime} \mathrm{d}$ have $\operatorname{Pr}_{\left(x_{2}, \ldots, x_{k}\right) \sim \mathcal{U}(S)}\left[g\left(x_{2}, \ldots, x_{k}\right)=x_{1}\right]=2^{-m}$ for every $x_{1}$ and every $S$. The mixing property in Definition 3.14 says that $F$ looks "almost random" on any "large" cylinder intersection.

Remark 3.15. In the condition $|I| \geq|Z| \cdot 2^{-2 m}$, the choice of $-2 m$ as an exponent might seem arbitrary. As it is, Definition 3.14 allows us to prove Theorem 3.17. But, in fact, for both the construction in this section, and for the construction in Section 3.5.2, we could prove an even stronger mixing property, one that would apply to even smaller rectangles: for every constant $\alpha$, for large enough $N$ (how large could depend on $\alpha$ ), for every cylinder intersection $I$ with $|I| \geq|Z| \cdot 2^{-\alpha m}$, the same property as above would be required of $I$. However, we do not know how to use this stronger mixing property to prove a larger lower bound than the one in Theorem 3.17 .

In the special case when $F$ is the function family from Definition 3.13, we have $k=$ 3 and $F_{3, N}$ has effective domain $\mathbb{F}_{2^{m}} \times \mathcal{H}^{n, m} \times \mathbb{F}_{2^{n}}$, so we can visualize $Z=\mathcal{H}^{n, m} \times \mathbb{F}_{2^{n}}$

\footnotetext{
${ }^{2}$ We're omitting $\{\perp\}$ from the range of $g$ in this informal statement.
} 
as a 2-dimensional matrix in which rows correspond to hash functions $h \in \mathcal{H}^{n, m}$, columns correspond to inputs $x^{\prime} \in \mathbb{F}_{2^{n}}$, and the entry at row $h$ and column $x^{\prime}$ is $Z_{h, x^{\prime}}=$ $h\left(x^{\prime}\right) \in \mathbb{F}_{2^{m}}$. Furthermore, cylinder intersections in 2 dimensions are rectangles. With this interpretation, the mixing property says that for every large rectangle $R \subseteq Z$, more precisely, when $|R| \geq|Z| \cdot 2^{-2 m}$, the number of $y$-entries in $R$ is at most twice the expected number if $R$ was filled at random with values from $\mathbb{F}_{2^{m}}$. The fact that $F$ has this property follows directly from the Hash Mixing Lemma in [MNT93].

Lemma 3.16. The function family F from Definition 3.13 has the mixing property from Definition 3.14 .

Proof of Lemma 3.16. Since $\mathcal{H}^{n, m}$ is a universal family of hash functions, by Lemma 13 in [MNT93], for every rectangle $R \subseteq Z$ and for every $y \in \mathbb{F}_{2^{m}}$, when $\left(h, x^{\prime}\right)$ are drawn uniformly from $R$, we have

$$
\operatorname{Pr}_{\left(h, x^{\prime}\right) \sim \mathcal{U}(R)}\left[h\left(x^{\prime}\right)=y\right] \leq \frac{1}{\left|\mathbb{F}_{2^{m}}\right|}+\left(\frac{\left|\mathcal{H}^{n, m}\right|}{|R| \cdot\left|\mathbb{F}_{2^{m}}\right|}\right)^{1 / 2}=2^{-m}+\left(\frac{|Z|}{|R|} \cdot 2^{-n-m}\right)^{1 / 2} .
$$

When $|R| \geq|Z| \cdot 2^{-2 m}$, as in Definition 3.14, we have $\operatorname{Pr}\left[h\left(x^{\prime}\right)=y\right] \leq 2^{-m}+2^{-n / 2+m / 2}$. Since $n / 2 \geq 3 m / 2$ for large enough $N$, we get $\operatorname{Pr}\left[h\left(x^{\prime}\right)=y\right] \leq 2^{-m+1}$.

Evidence Towards a Conjecture. The following is not a precise argument, but we consider it evidence towards the conjecture that for a function that has the mixing property above, the deterministic communication complexity of is large.

Let $F$ be the function family from Definition 3.13 . So $F_{3, N}$ has effective domain $\mathbb{F}_{2^{m}} \times \mathcal{H}^{n, m} \times \mathbb{F}_{2^{n}}$. Let $Z=\mathcal{H}^{n, m} \times \mathbb{F}_{2^{n}}$. Let $d=D_{3}\left(F_{3, N}\right)$ be the cost of the best deterministic protocol for $F_{3, N}$. By Lemma 3.8 , there exists a $2^{d}$-coloring $\chi$ of $Z$ and there are $2^{d+m}$ rectangles $R_{\ell, y} \subseteq Z$, for $\ell \in\left[2^{d}\right]$ and $y \in \mathbb{F}_{2^{m}}$, such that

$$
\forall\left(y, h, x^{\prime}\right) \in \mathbb{F}_{m} \times \mathcal{H}^{n, m} \times \mathbb{F}_{n}: \quad\left(h, x^{\prime}\right) \in R_{\chi\left(h, x^{\prime}\right), y} \Leftrightarrow h(x)=y .
$$

In keeping with the matrix interpretation of $Z$, we say that $\left(h, x^{\prime}\right) \in Z$ is an $(\ell, y)$-entry in $Z$ if and only if $\chi\left(h, x^{\prime}\right)=\ell$ and $h\left(x^{\prime}\right)=y$. For a subset $S \subseteq Z$, let $\#_{(\ell, y)}(S)$ denote 
the number of $(\ell, y)$-entries in $S$ and let $\rho_{(\ell, y)}(S)=\#_{(\ell, y)}(S) /|S|$ denote the density of $(\ell, y)$-entries in $S$. We use the notation $(\ell, \cdot)$ and $(; y)$ to refer to all entries with color $\ell$ and all entries with value $y$, respectively. For every $(\ell, y) \in\left[2^{d}\right] \times \mathbb{F}_{2^{m}}$, let

$$
B_{\ell, y}=R_{\ell, y} \backslash \bigcup_{y^{\prime} \neq y} R_{\ell, y^{\prime}}
$$

We call this set the boundary of the rectangle $R_{\ell, y}$. Note that, by Definition 3.9 of the alternative representation of $F$, all $(\ell, y)$-entries in $Z$ are in $B_{\ell, y}$.

The entries in $Z$ are colored with $2^{d}$ colours. Let red be a "popular" color, in the sense that $\rho_{\left(\mathrm{red}_{i}\right)}(Z) \geq 1 / 2^{d}$. When $y$ is chosen uniformly at random from $\mathbb{F}_{2^{m}}$,

$$
\begin{aligned}
& \underset{y \sim \mathcal{U}\left(\mathbb{F}_{2^{m}}\right)}{\mathrm{E}}\left[\#_{(\cdot y)}\left(B_{\text {red, }, y}\right)\right] \geq \mathrm{E}\left[\#_{(\operatorname{red}, y)}\left(B_{\text {red }, y}\right)\right] \quad\left(\text { might contain }\left(\ell^{\prime}, y\right) \text { entries for } \ell^{\prime} \neq \ell\right) \\
& =\mathrm{E}\left[\#_{(\operatorname{red}, y)}(Z)\right] \quad\left(\text { all }(\text { red }, y) \text { entries in } Z \text { are in } B_{\text {red }, y}\right) \\
& =\frac{\#\left(\mathrm{red}_{;}\right)}{2^{m}} \\
& \geq \frac{|Z|}{2^{m+d}} \text {. }
\end{aligned}
$$

Furthermore, for various $y$, the sets $B_{\text {red }, y}$ are disjoint, so

$$
\underset{y \sim \mathcal{U}\left(\mathbb{F}_{2^{m}}\right)}{\mathrm{E}}\left[\left|B_{\text {red }, y}\right|\right] \leq \frac{|Z|}{2^{m}}
$$

If we fixed a $y$ for which both quantities above are within a constant factor of their respective expectations (this is imprecise, but we believe it could be made precise), we would obtain a pair $($ red, $y)$ such that

$$
\rho_{(; y)}\left(B_{\mathrm{red}, y}\right)=\frac{\#(, y)\left(B_{\mathrm{red}, y}\right)}{\left|B_{\mathrm{red}, y}\right|} \geq \Omega\left(\frac{|Z|}{2^{m+d}} \cdot \frac{2^{m}}{|Z|}\right) \geq \Omega\left(\frac{1}{2^{d}}\right) .
$$

However, by mixing property in Definition 3.14 if $R_{\text {red }, y}$ is large enough,

$$
\rho_{(, y)}\left(R_{\mathrm{red}, y}\right) \leq O\left(\frac{1}{2^{m}}\right) .
$$

The smaller $d$ is, the larger the gap between these numbers. The reason this situation does not immediately translate into a lower bound for $d$ in terms of $m$ is that $B_{\text {red, } y}$ is far from a rectangle, so its density of $(; y)$-entries could be much larger than that of the rectangle $R_{\text {red, } y}$. However, we consider this evidence that $d$ might have to be large. 
A Weaker Lower Bound. In the following Theorem, we show that every family of graph functions $F=\left(F_{k, N}\right)_{N}$, such that $F_{k, N}:\left(\{0,1\}^{N}\right)^{k} \rightarrow\{0,1\}$ has effective domain $X_{1} \times \ldots \times X_{k}$, and that satisfies the mixing property from Definition 3.14 has deterministic communication complexity at least logarithmic in $m=m(N)=\log \left|X_{1}\right|$, the number of bits required to represent an element from the effective domain for player 1.

We describe the proof idea for the case of $k=3$ players, when we can view $Z$ as a matrix, but the proof itself works in general for $k \geq 3$. Let $d=D_{3}\left(F_{3, N}\right)$ and consider the $2^{d}$-coloring $\chi$ of the matrix $Z$ given by Lemma 3.8 . The proof proceeds by inductively decreasing the number of colors available and shrinking the matrix. During each step, we introduce a number of "holes" in the matrix (entries that are colored in the original matrix with one of the removed colors). We show that eventually there are no colors left to use, but the matrix still does not consist only of holes.

To see the limitation of this technique, observe that even if we were able to shrink the matrix by, say, a factor of 2 in order to remove every color (the factor we use below is larger), because there are $2^{d}$ colors to remove, at the end we would have shrunk the matrix by a factor of $2^{2^{d}}$. Since $Z$ has size at most $2^{(k-1) N}$, this technique can only produce lower bounds of the form $d \geq \Omega(\log N)$.

Theorem 3.17. Let $F=\left(F_{k, N}\right)_{N}$ be a family of functions that satisfies the mixing property in Definition 3.14. Let $X_{1} \times \ldots \times X_{k}$ be the effective domain of $F_{k, N}$ and let $m:=\log \left|X_{1}\right|$. Then, $D_{k}\left(F_{k, N}\right) \geq \Omega(\log m)$.

Proof of Theorem 3.17. Let $d=D_{k}\left(F_{k, N}\right)$, let $Z:=X_{2} \times \cdots \times X_{k}$, and let $g: Z \rightarrow$ $X_{1} \cup\{\perp\}$ be the unique function such that $F\left(y, x_{2}, \ldots, x_{k}\right)=1$ if and only if $y=$ $g\left(x_{2}, \ldots, x_{k}\right)$. By Lemma 3.8 , there is a $2^{d}$-coloring $\chi$ of $Z$ and there are $2^{d+m}$ cylinder intersections $I_{\ell, y}$, for $(\ell, y) \in\left[2^{d}\right] \times X_{1}$, such that

$$
\forall\left(y, x_{2}, \ldots, x_{k}\right) \in X_{1} \times Z, \quad\left(x_{2}, \ldots, x_{k}\right) \in I_{\chi\left(x_{2}, \ldots, x_{k}\right), y} \Leftrightarrow g\left(x_{2}, \ldots, x_{k}\right)=y .
$$

We say that a point $\left(x_{2}, \ldots, x_{k}\right)$ has color $\chi\left(x_{2}, \ldots, x_{k}\right)$ and value $g\left(x_{2}, \ldots, x_{k}\right)$. For a set 
$S \subseteq Z$, let $\#_{(\ell, y)}(S)$ denote the number of points $\left(x_{2}, \ldots, x_{k}\right) \in S$ with color $\ell$ and value $y$.

Assume there exists some $\epsilon<1$ such that for all $m, d \leq \epsilon \cdot \log m$. We will derive a contradiction.

For large enough $m$, we prove by induction that for all $0 \leq i \leq 2^{d}$, there exists a cylinder intersection $I_{i} \subseteq Z$ and a set of "holes" $H_{i} \subseteq I_{i}$ such that:

- $\left|I_{i}\right| \geq|Z| \cdot 2^{-i(d+2)} ; 3$

- $\left|H_{i}\right| \leq i \cdot|Z| \cdot 2^{-m+1} ;$ and

- the initial coloring induces a coloring of the points in $I_{i} \backslash H_{i}$ with $2^{d}-i$ colors. Assuming we have established this inductive statement, letting $i=2^{d}$, we see that

$$
\begin{array}{rlrl}
\left|I_{2^{d}}\right| & \geq|Z| \cdot 2^{-2^{d}(d+2)} \quad & & \text { (from inductive statement) } \\
& \geq|Z| \cdot 2^{-m^{\epsilon}(\epsilon \log m+2)} \quad & \text { (since } d<\epsilon \log m)
\end{array}
$$

Also,

$$
\begin{aligned}
\left|H_{2^{d}}\right| & \leq|Z| \cdot 2^{-m+d+1} \quad \text { (from inductive statement) } \\
& \leq|Z| \cdot 2^{-m+\epsilon \log m+1}
\end{aligned}
$$

For large enough $m,-m^{\epsilon}(\epsilon \log m+2)>-m+\epsilon \log m+1$, so $\left|H_{2^{d}}\right|<\left|I_{2^{d}}\right|$ and $I_{2^{d}} \backslash$ $H_{2^{d}} \neq \varnothing$. But there are no colours left for the points in $I_{2^{d}} \backslash H_{2^{d}}$, which means they were uncoloured in the original colouring $\chi$. This is a contradiction.

We now prove the inductive statement. For $i=0$, let $I_{0}=Z$ and let $H_{0}=\varnothing$. By definition, $\chi$ is a coloring of $Z=I_{0} \backslash H_{0}$ with $2^{d}=2^{d}-0$ colors.

Next, assume the inductive statement is true for some $0 \leq i<2^{d}$. We have

$$
\left|I_{i} \backslash H_{i}\right| \geq|Z| \cdot\left(2^{-i(d+2)}-i \cdot 2^{-m+1}\right)>|Z| \cdot\left(2^{-i(d+2)}-2^{-m+d+1}\right)>|Z| \cdot 2^{-i(d+2)-1},
$$

\footnotetext{
${ }^{3}$ This corresponds to a shrinking factor of $2^{d+2}$ at every step.
} 
where in the last inequality we used $m-d-1>m^{\epsilon}(\epsilon \cdot \log m+2)>i(d+2)$, for large enough $m$.

Let $(\ell, y)$ be the most popular color-value pair in $I_{i} \backslash H_{i}$. There are at most $2^{m+d}$ such pairs, so

$$
\#_{(\ell, y)}\left(I_{i} \backslash H_{i}\right) \geq\left|I_{i} \backslash H_{i}\right| \cdot 2^{-m-d} \geq|Z| \cdot 2^{-i(d+2)-1-m-d}=|Z| \cdot 2^{-(i+1)(d+2)-m+1} .
$$

Let $I_{i+1}=I_{i} \cap I_{\ell, y}$, which is a cylinder intersection in $Z$ because both $I_{i}$ and $I_{\ell, y}$ are. By the property of the coloring $\chi$ in Lemma 3.8 , all points in $Z$ with color-value $(\ell, y)$ are in $I_{\ell, y}$. Hence,

$$
\left|I_{i+1}\right| \geq \#_{(\ell, y)}\left(I_{i} \backslash H_{i}\right) \geq|Z| \cdot 2^{-(i+1)(d+2)-m+1} .
$$

Note that $(i+1)(d+2)-1 \leq m^{\epsilon}(\epsilon \log m+2)-1<m$, so $\left|I_{i+1}\right| \geq|Z| \cdot 2^{-2 m}$. Then, by the mixing property in Definition $3.14, \#_{(, y)}\left(I_{i+1}\right) /\left|I_{i+1}\right| \leq 2^{-m+1}$. Hence, we can improve the lower bound on $\left|I_{i+1}\right|$ as follows

$$
\begin{aligned}
\left|I_{i+1}\right| & \geq 2^{m-1} \cdot \#_{(\cdot y)}\left(I_{i+1}\right) \\
& \geq 2^{m-1} \cdot \#_{(\ell, y)}\left(I_{i+1}\right) \\
& \geq 2^{m-1} \cdot \#_{(\ell, y)}\left(I_{i} \backslash H_{i}\right) \\
& \geq 2^{m-1} \cdot|Z| \cdot 2^{-(i+1)(d+2)-m+1} \\
& =|Z| \cdot 2^{-(i+1)(d+2)},
\end{aligned}
$$

establishing the first part of the inductive statement.

Let $H_{i+1}=H_{i} \cup\left\{\left(x_{2}, \ldots, x_{k}\right) \in I_{i+1} \mid \chi\left(x_{2}, \ldots, x_{k}\right)=\ell\right\}$. By induction hypothesis, points in $I_{i} \backslash H_{i}$ are colored with at most $2^{d}-i$ colors. Since $\ell$ is no longer available, we see that all points left in $I_{i+1} \backslash H_{i+1}$ must now be colored with at most $2^{d}-i-1$ colors, establishing the third part of the inductive statement.

Finally, by the property of $\chi$ in Lemma 3.8 , all points in $I_{\ell, y}$ that have color $\ell$ must have value $y$. In the entire set $Z$, by the mixing property, there are at most $|Z| \cdot 2^{-m+1}$ points with value $y$. Then, $\left|H_{i+1}\right| \leq\left|H_{i}\right|+|Z| \cdot 2^{-m+1} \leq(i+1) \cdot|Z| \cdot 2^{-m+1}$, establishing the second part of the inductive statement. 
Corollary 3.18. The function family F from Definition 3.13 satisfies $D_{3}\left(F_{3, N}\right) \geq \Omega(\log N)$. Furthermore, F separates public-coin and private-coin randomized protocols, as $R_{3}^{\mathrm{pub}}\left(F_{3, N}\right) \leq$ $O(1)$ and $R_{3}\left(F_{3, N}\right) \geq \Omega(\log \log N)$.

Proof of Corollary 3.18, By Lemma 3.16, $F$ has the mixing property. By Theorem 3.17, $D_{3}\left(F_{3, N}\right) \geq \Omega(\log m)$. By definition of $F, m=(N / 3)^{1 / 2}$, so $D_{3}\left(F_{3, N}\right) \geq \Omega(\log N)$. Since $F_{3, N}$ is graph, by Lemma $3.4, R_{3}^{\text {pub }}\left(F_{3, N}\right) \leq O(1)$. Finally, by Lemma 3.12, $R_{3}\left(F_{3, N}\right) \geq \Omega(\log \log N)$.

\subsubsection{A Function Family for Three or More Players}

In the case of $k \geq 3$ players, the family of graph functions we construct is based on the Generalized Inner Product function over a finite field. It turns out that the crucial "mixing property" in Definition 3.14 follows quite easily from a bound on the "strong discrepancy" of the Generalized Inner Product function obtained by [BHK01].

Recall the definition of discrepancy for a boolean function $f: Z \rightarrow\{0,1\}$ on a set $S \subseteq Z$ from Section 2.1. In particular, if we consider the uniform distribution $\mathcal{U}$ on $Z$, we have:

$$
\operatorname{disc}(f, S)=\operatorname{disc}_{\mathcal{U}}(f, S)=\frac{1}{|Z|} \cdot|| f^{-1}(0) \cap S|-| f^{-1}(1) \cap S||
$$

A natural generalization for the notion of discrepancy for a non-boolean function $f$ : $Z \rightarrow B$ on a set $S \subseteq Z$ is the strong discrepancy, defined in [BHK01]. This measure considers all possible function values $y \in B$, and it reflects the largest difference between the actual number of $y$-inputs in $S$ and the average number of such inputs if $f$ were 
perfectly balanced on $S$. Formally, 4

$$
\begin{aligned}
\operatorname{disc}^{*}(f, S) & :=\max _{y \in B} \frac{1}{|Z|} \cdot|| f^{-1}(y) \cap S\left|-\frac{|S|}{|B|}\right| \\
& =\max _{y \in B} \frac{|S|}{|Z|} \cdot\left|\operatorname{Pr}_{z \sim \mathcal{U}(S)}[f(z)=y]-\frac{1}{|B|}\right|
\end{aligned}
$$

It is not hard to see that strong discrepancy is connected to the mixing property from Definition 3.14. Before making this connection formal, we define the function we will be using.

Let $n, m \geq 1$, and $p \geq 2$. Let $V=\left(\mathbb{F}_{2^{m}}\right)^{n}$. We view $V$ as an $n$-dimensional vector space over the field $\mathbb{F}_{2^{m}}$. Recall the definition of the Field Generalized Inner Product function $\mathrm{FGIP}_{k, n}^{\mathbb{F}_{2 m}}: V^{k} \rightarrow \mathbb{F}_{2^{m}}$ from Section 2.3. [BHK01] show the following upper bound on its strong discrepancy.

Fact 3.19 ([BHK01] $\left.]^{5}\right)$. For every cylinder intersection $I \subseteq V^{p}$,

$$
\operatorname{disc}^{*}\left(\operatorname{FGIP}_{k, n}^{\mathbb{F}_{2^{m}}}, I\right) \leq\left(1-\frac{1}{2^{m}}\right) \cdot\left(1-\left(1-\frac{1}{2^{m}}\right)^{k-1}\right)^{n \cdot 2^{1-k}}
$$

For $k \geq 3$ players, consider the following graph function.

Definition 3.20. Let $k=k(N)$ be a function satisfying $3 \leq k \leq(1 / 9) \cdot \log N$, for all sufficiently large $N$. Let $F=\left(F_{k, N}\right)_{N}$ be the function family where $F_{k, N}$ is defined as follows. Let $n:=N^{1 / 2}$, let $m:=n^{1 / 2}=N^{1 / 4}$. On input $\left(x_{1}, x_{2}, \ldots, x_{k}\right), F_{k, N}$ is 0 unless:

(i) $x_{1}=y \circ 0^{N-m}$ for some $y \in \mathbb{F}_{2^{m}}$,

(ii) for every $2 \leq i \leq k, x_{i}=z_{i, 1} \circ \ldots \circ z_{i, n} \circ 0^{N-m n}$ where $z_{i}=\left(z_{i, 1}, \ldots, z_{i, n}\right) \in V$, and

\footnotetext{
${ }^{4}$ Observe that strong discrepancy when $|B|=2$ and regular discrepancy are off by a factor of 2 . To get them to agree, we'd have to multiply strong discrepancy by $|B|$. In here, we simply use the definition given in [BHK01].

${ }^{5}$ In [BHK01], this fact is not stated as a stand-alone Lemma, but it is proved and used as part of the proof of Corollary 4.12.
} 
(iii) $y=\operatorname{FGIP}_{k-1, n}^{\mathbb{F}_{2 m}}\left(z_{2}, \ldots, z_{k}\right)$.

Note that the effective domain of $F_{k, N}$ is $\mathbb{F}_{2^{m}} \times V \times \ldots \times V$.

We claim that the small strong discrepancy of $\operatorname{FGIP}_{k-1, n}^{\mathbb{F}_{2 m}}$ implies that $F$ satisfies the mixing property from Definition 3.14 .

Lemma 3.21. The function family F from Definition 3.20 has the mixing property from Definition 3.14 .

Proof of Lemma 3.21. Let $I \subseteq V^{k-1}$ be a cylinder intersection with $|I| \geq\left|V^{k-1}\right| \cdot 2^{-2 m}$ and let $y \in \mathbb{F}_{2^{m}}$. We want to show that

$$
\operatorname{Pr}_{\left(z_{2}, \ldots, z_{k}\right) \sim \mathcal{U}(I)}\left[\operatorname{FGIP}_{k-1, n}^{\mathbb{F}_{2 m}}\left(z_{2}, \ldots, z_{k}\right)=y\right] \leq 2^{-m+1}
$$

Writing $z$ for $\left(z_{2}, \ldots, z_{k}\right)$ and $g$ for $\operatorname{FGIP}_{k-1, n^{\prime}}^{\mathbb{F}_{2^{m}}}$, from the definition of strong discrepancy we get

$$
\begin{aligned}
\left|\operatorname{Pr}_{z \sim \mathcal{U}(I)}[g(z)=y]-\frac{1}{2^{m}}\right| & \leq \frac{\left|V^{k-1}\right|}{|I|} \cdot \operatorname{disc}^{*}(g, I) \\
& \leq 2^{2 m} \cdot \operatorname{disc}^{*}(g, I)
\end{aligned}
$$

By Fact 3.19 (with $p=k-1$ ),

$$
\begin{array}{rlrl}
\operatorname{disc}^{*}(g, I) & \leq\left(1-\frac{1}{2^{m}}\right) \cdot\left(1-\left(1-\frac{1}{2^{m}}\right)^{k-2}\right)^{n \cdot 2^{2-k}} & \\
& \leq\left(1-\left(1-\frac{1}{2^{m}}\right)^{k-2}\right)^{n \cdot 2^{2-k}} & & \text { (since } m \geq 1) \\
& \leq \exp \left(-\left(1-\frac{1}{2^{m}}\right)^{k-2} \cdot n \cdot 2^{2-k}\right) & & \text { (using } \left.1-x \leq e^{-x}\right) \\
& \leq \exp \left(-\frac{1}{2^{k-2}} \cdot n \cdot 2^{2-k}\right) & & \text { (since } \left.1-1 / 2^{m} \geq 1 / 2\right) \\
& =2^{-16(\log e) n \cdot 2^{-2 k}} &
\end{array}
$$


Putting everything together,

$$
\begin{aligned}
\operatorname{Pr}_{z \sim \mathcal{U}(I)}[g(z)=y] & \leq \frac{1}{2^{m}}+2^{2 m} \cdot \operatorname{disc}^{*}(g, I) \\
& \leq 2^{-m}+2^{2 m-16(\log e) n \cdot 2^{-2 k}} \\
& \leq 2^{-m}+2^{-m}=2^{-m+1} \quad(\text { for large enough } N)
\end{aligned}
$$

Above, we need $n \geq\left(3 \cdot m \cdot 2^{2 k}\right) /(16 \cdot \log e)$. Since $\log n=(1 / 2) \cdot \log N, \log m=(1 / 4)$. $\log N$ and $2 k \leq(2 / 9) \cdot \log N<(1 / 4) \cdot \log N$, the inequality holds for large enough $N$.

Corollary 3.22. The function family $F$ from Definition 3.20 has $D_{k}\left(F_{k, N}\right) \geq \Omega(\log N)$. Furthermore, F separates public-coin and private-coin randomized protocols, as $R_{k}^{\mathrm{pub}}\left(F_{k, N}\right) \leq$ $O(1)$ and $R_{k}\left(F_{k, N}\right) \geq \Omega(\log \log N)$.

Proof of Corollary 3.22. By Lemma 3.21 and Theorem 3.17, $D_{k}\left(F_{k, N}\right) \geq \Omega(\log m)$. Since $m=N^{1 / 4}, D_{k}\left(F_{k, N}\right) \geq \Omega(\log N)$. Since $F_{k, N}$ is graph, by Lemma $3.4, R_{k}^{\text {pub }}\left(F_{k, N}\right) \leq$ $O(1)$. Finally, by Lemma 3.12, $R_{k}\left(F_{k, N}\right) \geq \Omega(\log \log N)$.

\subsection{Discussion}

In this chapter, we proved that there exists a function family $F=\left(F_{k, N}\right)_{N}$ which has efficient randomized protocols, but no efficient deterministic protocols. To do this, we considered the class of "graph" functions $f^{g}:\left(\{0,1\}^{N}\right)^{k} \rightarrow\{0,1\}$ defined by $f^{g}\left(x_{1}, x_{2}, \ldots, x_{k}\right)=1$ if and only if $x_{1}=g\left(x_{2}, \ldots, x_{k}\right)$, where $g$ is a function with domain $\left(\{0,1\}^{N}\right)^{k-1}$ and range $\{0,1\}^{m}$ for some $m \leq N$. We proved that for every $g, f^{g}$ reduces easily to 2-player Equality, which has an efficient randomized protocol. Then, we showed that every $c$-bit deterministic protocol for $f^{g}$ can be transformed into an "oblivious-and-check" protocol, where player 1 first communicates $c$ bits, and then 
the remaining players simultaneously send 1 bit each. We gave a combinatorial interpretation of protocols of this type in terms of colourings and cylinder intersections. Using this interpretation, we were able to apply a counting argument to show that, when $m \approx N / 2$ and $c \approx N / 2$, there are more functions $g:\left(\{0,1\}^{N}\right)^{k-1} \rightarrow\{0,1\}^{m}$ (and hence, $f^{g}$ ) than there are oblivious-and-check protocols where player 1 communicates $c$ bits. From this, it follows that for some $g$, the best deterministic protocol for $f^{g}$ requires $\approx N / 2$ bits of communication.

The original discrepancy method of [BNS92] for proving lower bounds in the NOF model automatically proves lower bounds for randomized protocols, making it unsuitable for separating deterministic and randomized protocols. It is an open problem to adapt the recent "generalized" discrepancy method [Kla07, Raz03, She08a, LS08, CA08] to separate deterministic and randomized protocols. Apart from the results in this chapter, the only previously known non-discrepancy-based lower bounds for deterministic protocols are based on Ramsey theory [CFL83, Pud03], and the actual lower bounds they obtain are very weak (only super-constant, $\omega(1)$ ). The results in here are the first, and to this date, the only, strong $\left(n^{\Omega}(1)\right)$ deterministic lower bounds for functions with efficient randomized protocols.

Perhaps surprisingly, some of the technical arguments we use in this paper bear a similarity to the ones in [BHK01]. In that paper, the goal was to obtain lower bounds for $k$-player NOF protocols "with help". These are protocols for non-boolean functions $g:\left(\{0,1\}^{N}\right)^{k} \rightarrow\{0,1\}^{m}$, where at the start of the protocol, the players receive an $(m-1)$-bit message from a benevolent helper that sees the entire input. Thus, potentially, the helper can give away the first $m-1$ bits of the output of $g$, and the goal of the protocol is to retrieve the remaining bit. The result proved in that paper is discrepancy-based, so it works even for randomized protocols with help, and it says that there are functions for which the best protocol with help requires $\Omega\left(N / c^{k}\right)$ bits of communication. 
Even though the results in [BHK01] and the ones presented in this chapter seem rather different, it turns out that they are somewhat similar at the technical level. We have seen that if a function $f^{g}$ has a deterministic protocol of $\operatorname{cost} c$, then there is a $2^{c}$ colouring of the inputs to $g$, and cylinder intersections $I_{\ell, x}$ such that, for every $\ell$, $\left(I_{\ell, x} \mid x \in\{0,1\}^{m}\right)$ partition the $\ell$-coloured inputs to $g$. In contrast, if a function $g$ has a protocol with $c$ bits of help and communication cost $s$, it can be shown that there are cylinder intersections $T_{\ell, x}$ such that, for every $\ell,\left(T_{\ell, x} \mid x \in\{0,1\}^{s}\right)$ partition the entire set of inputs to $g$. Because the partitioning is more strict in the case of [BHK01], they were able to connect the cost of a protocol with help directly to the strong discrepancy of the function being computed. In our case, we were not able to make this connection because the sets corresponding to colour $\ell$ can freely intersect on inputs which are not $\ell$-coloured.

We leave as open problem the separation of deterministic and randomized protocols by an explicit (say, polynomial time computable) function. It would also be interesting to study whether counting arguments can be used to separate other types of protocols, for example, randomized from nondeterministic. Such a separation exists, and is the subject of the next chapter. However, the dependency of this separation on the number of players is limited to $k<\log n$ because of its ultimate reliance on the discrepancy method [BNS92]. Potentially, a counting argument might avoid this limitation. 


\section{Chapter 4}

\section{Randomized vs. Nondeterministic}

\section{Complexity}

Until recently, it was far from clear how to obtain communication lower bounds in the NOF model of communication for any function $F$ with efficient nondeterministic protocols. The difficulty can be described as follows. The standard method for obtaining lower bounds is the so-called discrepancy method introduced in [BNS92], and rewritten in [CT93, Raz00]. This is a method for showing that certain functions $F$ have exponentially small $\left(2^{-N^{\Omega(1)}}\right)$ correlation 1 with efficient deterministic protocols. Using Yao's min-max principle, this immediately implies that $F$ does not have an efficient randomized protocol. The drawback of this method is that, although for the conclusion that $F$ does not have an efficient randomized protocol, it is enough to show that the correlation of $F$ with efficient deterministic protocols is strictly less than 1 , the discrepancy method actually proves a stronger, exponentially small, correlation bound. However, it is not hard to show that every function that has an efficient nondeterministic protocol also has noticeable $\left(\geq 2^{-\log (1)} N\right)$ correlation with an efficient deterministic protocol, and thus, this method does not seem useful for separating nondeterministic

\footnotetext{
${ }^{1}$ With respect to some probability distribution which is, in general, not uniform.
} 
from randomized protocols.

In a recent line of work, surveyed in [She08a], these difficulties were overcome to obtain a lower bound for a function with an efficient nondeterministic protocol: the Set Intersection function [LS08, CA08]. The starting point is the work by Sherstov [She09, She08b] which applied the original discrepancy method [BNS92] in a more general way for the 2-player model in order to overcome the above difficulties. This generalized discrepancy method was then adapted to the NOF model with more players $(k \gg 2)$ in [LS08, CA08].

The high-level idea of the method is as follows. Suppose that we want to prove that some specific function $F$ does not have efficient randomized protocols. The idea is to come up with another function $F^{\prime}$ and a distribution $\lambda$ such that:

(1) $F$ and $F^{\prime}$ have high (constant) correlation, say $F$ and $F^{\prime}$ disagree on at most $1 / 10$ mass of the inputs with respect to $\lambda$; and

(2) $F^{\prime}$ has exponentially small $\left(2^{-N^{\Omega(1)}}\right)$ correlation with efficient deterministic protocols with respect to $\lambda$.

The combination of (1) and (2) easily implies that $F$ also has correlation at most $1 / 10+$ $2^{-N^{\Omega(1)}}<1$ with efficient deterministic protocols, which gives the desired lower bound for $F$. This method is useful because for $F^{\prime}$ we can use the original discrepancy method, and yet the correlation of $F$ with efficient deterministic protocols is not shown to be exponentially small, only bounded away from 1 by a constant. Thus, it is conceivable that $F$ has an efficient nondeterministic protocol, and in fact this is the case in [LS08, CA08] and in this work.

Although a similar framework was present in [Kla07, Raz03], it is the work by Sherstov [She09, She08b] that found a way to successfully apply it to functions $F$ with efficient nondeterministic protocols. For this, two main ideas were introduced in [She09, She08b], and generalized to the NOF model in [LS08, CA08]. (We present them specialized for our needs, see [She08a] for a broader view.) The first is to con- 
sider a special class of functions Lift $(\mathrm{OR}, \phi)$ with efficient nondeterministic protocols. These are obtained by combining the "base" function OR on $m$ bits with a "selection" function $\phi$, as described next. It is convenient to think of Lift $(\mathrm{OR}, \phi)$ as a function on $(k+1) \cdot N$ bits distributed among $k+1$ players as follows: player 0 receives an $N$-bit vector $x$, while player $i$, for $1 \leq i \leq k$, gets an $N$-bit vector $y_{i}$. The selection function $\phi$ takes as input $y_{1}, \ldots, y_{k}$ and outputs an $m$-bit subset of $\{1, \ldots, N\}$. We view $\phi$ as selecting $m$ bits of the input $x$ of player 0 , denoted $x \mid \phi\left(y_{1}, \ldots, y_{k}\right)$. Lift $(\mathrm{OR}, \phi)$ outputs the value of OR on those $m$ bits of $x$ :

$$
\operatorname{Lift}(\mathrm{OR}, \phi)\left(x, y_{1}, \ldots, y_{k}\right):=\mathrm{OR}\left(x \mid \phi\left(y_{1}, \ldots, y_{k}\right)\right) \text {. }
$$

The second idea is to apply to such a function $F=\operatorname{Lift}(\mathrm{OR}, \phi)$ a certain orthogonality principle, in order to produce a function $F^{\prime}$ that satisfies (1) and (2) above. The structure of Lift $(\mathrm{OR}, \phi)$ is crucially exploited to argue that $F^{\prime}$ satisfies (2), and it is here that previous works required $k<\log \log N$ [Cha07, LS08, CA08].

Building upon this line of results, in this chapter, we present the second major contribution of this work, which extends the above separation between randomized and nondeterministic NOF communication protocols for up to $k^{\prime} \leq \delta \cdot \log N$ players, for every $\delta<1$.

Theorem 4.1. For every constant $\delta<1$ and for every increasing function $k^{\prime}=k^{\prime}(N)$ satisfying $k^{\prime} \leq \delta \cdot \log N$ for sufficiently large $N$, there exists an polynomial time computable function family $F=\left(F_{k^{\prime}, N}\right)_{N}$, where $F_{k^{\prime}, N}:\left(\{0,1\}^{N}\right)^{k^{\prime}} \rightarrow\{0,1\}$, such that:

- $R_{\mathrm{NOF}, k^{\prime}}\left(F_{k^{\prime}, N}\right) \geq N^{\Omega(1)} ;$ and

- $N_{\mathrm{NOF}, k^{\prime}}\left(F_{k^{\prime}, N}\right) \leq O(\log N)$.

In particular, $F$ witnesses the fact that, for $k^{\prime}$ as above, $\mathrm{NP}_{\mathrm{NOF}, k^{\prime}}^{\mathrm{cc}} \not \subset \mathrm{BPP}_{\mathrm{NOF}, k^{\prime}}^{\mathrm{cc}}$ (and thus, that $\left.\mathrm{RP}_{\mathrm{NOF}, k^{\prime}}^{\mathrm{cc}} \subsetneq \mathrm{NP}_{\mathrm{NOF}, k^{\prime}}^{\mathrm{cc}}\right)$. 
To prove Theorem 4.1, we start by noting that regardless of what selection function $\phi$ is chosen, $\mathrm{Lift}(\mathrm{OR}, \phi)$ has an efficient nondeterministic protocol: player 0 simply guesses an index $j$ that is one of the indices chosen by $\phi$ (it can do so because it knows the input to $\phi$ ) and then any of the other players can easily verify whether or not $x_{j}$ is 1 in that position. In previous work [LS08, CA08], $\phi$ is the bitwise And function, and this makes $\operatorname{Lift}(\mathrm{OR}, \phi)$ the Set Intersection function. In contrast, in this work, we choose the selection function $\phi$ uniformly at random and we argue that, for almost all $\phi$, Lift $(\mathrm{OR}, \phi)$ does not have an efficient randomized protocol.

The above argument gives a non-explicit separation, we're simply proving that there exists a function $\phi$ such that $\operatorname{Lift}(\mathrm{OR}, \phi)$ is hard for randomized NOF protocols, without saying anything in particular about $\phi$. To make it explicit, we derandomize the choice of $\phi$. Specifically, we note that the above argument goes through as long as $\phi$ is $2^{k}$-wise independent (where $k^{\prime}=k+1$ ), i.e. as long as $\phi$ comes from a distribution such that for every $2^{k}$ fixed inputs $\bar{y}^{1}, \ldots, \bar{y}^{k} \in\left(\{0,1\}^{N}\right)^{k}$ the values $\phi\left(\bar{y}^{1}\right), \ldots, \phi\left(\bar{y}^{2^{k}}\right)$ are uniform and independent (over the choice of $\phi$ ). Known constructions of such distributions [ABI86, CG89] only require about $N \cdot 2^{k}=N^{O(1)}$ random bits, which can be given as part of the input. Two things should perhaps be stressed. The first is that giving a description of $\phi$ as part of the input does not affect the lower bound in the previous paragraph, which turns out to hold even against protocols that depend on $\phi$. The second is that, actually, using $2^{k}$-wise independence seems to add the constraint $k<(1 / 2) \cdot \log N$; to achieve $k \leq \delta \cdot \log N$ for every $\delta<1$, we use a distribution on $\phi$ that is almost $2^{k}$-wise independent [NN93].

We begin in Section 4.1 by introducing several definitions and known facts. In Section 4.2, we prove a non-explicit version of Theorem 4.1. Finally, in Section 4.3, we prove the explicit version.

The work in this chapter appears in [DPV09]. 


\subsection{Preliminaries}

In this chapter, we only consider the NOF model of communication. We find it more convenient to denote the number of players by $k^{\prime}=k+1$ (rather than by $k$, as usual). Throughout, let $k=k(N)$ be a nondecreasing function that satisfies $k+1 \leq \delta \cdot \log N$ for all sufficiently large $N$. Let $m=m(N)$ be an increasing function, which we eventually set to be a sub-linear polynomial $m=N^{\alpha}$ for some constant $0<\alpha<1$.

For analytical purposes, it is more convenient to consider some boolean functions over the range $\{-1,+1\}$ rather than the usual $\{0,1\}$. In doing so, we think of -1 as representing true and 1 as representing false. Thus, for example the $m$-bit Or function OR $:\{0,1\}^{m} \rightarrow\{-1,+1\}$ is defined by $\operatorname{OR}\left(x_{1}, \ldots, x_{m}\right)=-1$ if and only if there exists $1 \leq i \leq m$ such that $x_{i}=1$.

\subsubsection{Lift Function}

We restrict our attention to analyzing the communication complexity of certain functions constructed from a base function $f_{m}:\{0,1\}^{m} \rightarrow\{-1,+1\}$, and a selection function $\phi$. One of the base functions we work with is the Or function.

Definition 4.2. Let $\phi_{k, N}:\left(\{0,1\}^{N}\right)^{k} \rightarrow\left(\begin{array}{c}{[N]} \\ m\end{array}\right)$ be a function that takes as input $k N$-bit strings $y_{1}, \ldots, y_{k}$ and outputs an $m$-element subset of $[N]$. Let $f_{m}:\{0,1\}^{m} \rightarrow\{-1,+1\}$ be a function. We construct a lifted function $\operatorname{Lift}\left(f_{m}, \phi_{k, N}\right):\left(\{0,1\}^{N}\right)^{k+1} \rightarrow\{-1,+1\}$ as follows. On input $\left(x, y_{1}, \ldots, y_{k}\right) \in\left(\{0,1\}^{N}\right)^{k+1}, \operatorname{Lift}\left(f_{m}, \phi_{k, N}\right)$ evaluates $\phi_{k, N}$ on the latter $k$ inputs to select a set of $m$ bits in $x$ and returns the value of $f_{m}$ on those $m$ bits. Formally,

$$
\operatorname{Lift}\left(f_{m}, \phi_{k, N}\right)\left(x, y_{1}, \ldots, y_{k}\right):=f_{m}\left(x \mid \phi_{k, N}\left(y_{1}, \ldots, y_{k}\right)\right)
$$

where for a set $S=\left\{i_{1}<\ldots<i_{m}\right\} \subseteq[N], x \mid S$ denotes the substring $x_{i_{1}}, \ldots, x_{i_{m}}$ of $x$ indexed by the elements in $S$. 
The inputs to $\operatorname{Lift}\left(f_{m}, \phi_{k, N}\right)$ are partitioned among $k+1$ players as follows: player 0 is given $x$ and, for all $1 \leq i \leq k$, player $i$ is given $y_{i}$.

\subsubsection{Correlation}

Let $f, g: X \rightarrow \mathbb{R}$ be two functions, and let $\mu$ be a distribution on $X$. We define the correlation between $f$ and $g$ under $\mu$ to be $\operatorname{corr}_{\mu}(f, g):=\mathrm{E}_{x \sim \mu}[f(x) g(x)]$. Let $\mathcal{G}$ be a class of functions $g: X \rightarrow \mathbb{R}$ (e.g. efficient communication protocols). We define the correlation between $f$ and $\mathcal{G}$ under $\mu$ to be $\operatorname{corr}_{\mu}(f, \mathcal{G}):=\max _{g \in \mathcal{G}} \operatorname{corr}_{\mu}(f, g)$. Note that, whenever $\mathcal{G}$ is closed under complements, which will always be the case in here, this correlation is non-negative. Whenever we omit mentioning a specific distribution when computing the correlation, it is to be assumed that we are referring to the uniform distribution, $\mathcal{U}$.

We manipulate correlation using the following simple facts.

Fact 4.3. For all functions $f, g: X \rightarrow\{-1,+1\}$ and for every distribution $\mu$ on $X$,

$$
\operatorname{corr}_{\mu}(f, g)=1-2 \cdot \operatorname{Pr}_{x \sim \mu}[f(x) \neq g(x)]
$$

Proof of Fact 4.3 .

$$
\begin{aligned}
\operatorname{corr}_{\mu}(f, g) & =\underset{x \sim \mu}{\mathrm{E}}[f(x) \cdot g(x)]=\sum_{x}(\mu \cdot f \cdot g)(x) \\
& =\sum_{x: f(x)=g(x)} \mu(x)+(-1) \cdot \sum_{x: f(x) \neq g(x)} \mu(x) \\
& =\operatorname{Pr}_{x \sim \mu}[f(x)=g(x)]-\operatorname{Pr}_{x \sim \mu}[f(x) \neq g(x)] \\
& =1-2 \cdot \operatorname{Pr}_{x \sim \mu}[f(x) \neq g(x)] .
\end{aligned}
$$

Fact 4.4. For all functions $f, g, h: X \rightarrow\{-1,+1\}$ and for every distribution $\mu$ on $X$,

$$
\operatorname{corr}_{\mu}(f, h) \leq \operatorname{corr}_{\mu}(g, h)+1-\operatorname{corr}_{\mu}(f, g) .
$$


Proof of Fact 4.4 .

$$
\begin{array}{rlrl}
\operatorname{corr}_{\mu}(f, h) & =\underset{x \sim \mu}{\operatorname{E}}[f(x) \cdot h(x)]=\sum_{x}(\mu \cdot f \cdot h)(x) \\
& =\sum_{x: f(x)=g(x)}(\mu \cdot g \cdot h)(x)-\sum_{x: f(x) \neq g(x)}(\mu \cdot g \cdot h)(x) \\
& =\sum_{x}(\mu \cdot g \cdot h)(x)-2 \cdot\left(\sum_{z: f(x) \neq g(x)}(\mu \cdot g \cdot h)(x)\right) \\
& =\operatorname{corr}_{\mu}(g, h)-2 \cdot\left(\sum_{z: f(x) \neq g(x)}(\mu \cdot g \cdot h)(x)\right) \\
& \leq \operatorname{corr}_{\mu}(g, h)+2 \cdot\left(\sum_{x: f(x) \neq g(x)} \mu(x)\right) & \\
& =\operatorname{corr}_{\mu}(g, h)+2 \cdot \operatorname{Pr}_{x \sim \mu}[f(x) \neq g(x)] & \\
& \left.=\operatorname{corr}_{\mu}(g, h)+1-\operatorname{corr}_{\mu}(f, g) \quad \quad \text { (by Fact } g \cdot h \geq-1\right)
\end{array}
$$

Definition 4.5. Let $\Pi_{k^{\prime}, N}^{c}$ be the class of all deterministic $k^{\prime}$-player NOF communication protocols with input from $\left(\{0,1\}^{N}\right)^{k^{\prime}}$, output in $\{-1,+1\}$, and cost at most $c$.

The following fact allows us to derive lower bounds on the randomized communication complexity of $F$ from upper bounds on the correlation between $F_{k^{\prime}, N}$ and the class $\Pi_{k^{\prime}, N}^{c}$.

Fact 4.6. Let $F_{k^{\prime}, N}:\left(\{0,1\}^{N}\right)^{k^{\prime}} \rightarrow\{-1,+1\}$ be a function. If there exists a distribution $\mu$ on $\left(\{0,1\}^{N}\right)^{k^{\prime}}$ such that $\operatorname{corr}_{\mu}\left(F_{k^{\prime}, N}, \Pi_{k^{\prime}, N}^{c}\right)<1 / 3$, then $R_{k^{\prime}}(F)>c$.

Proof of Fact 4.6. We prove the contrapositive. Assume $R_{k^{\prime}}\left(F_{k^{\prime}, N}\right) \leq c$. Let $\mu$ be any distribution. By Fact 2.6, $D_{k^{\prime}}^{\mu}\left(F_{k^{\prime}, N}\right) \leq c$. So, there exists a protocol $\pi$ for $F_{k^{\prime}, N}$ that has cost at most $c$ and error at most $1 / 3$ under $\mu$. Then $\pi \in \Pi_{k^{\prime}, N}^{c}$ and

$$
\begin{aligned}
\operatorname{corr}_{\mu}\left(F_{k^{\prime}, N}, \Pi_{k^{\prime}, N}^{c}\right) & \geq \operatorname{corr}_{\mu}\left(F_{k^{\prime}, N}, \pi\right) \\
& =1-2 \cdot \operatorname{Pr}_{x \sim \mu}[f(x) \neq \pi(x)] \quad \text { (by Fact 4.3) } \\
& \geq 1-2 \cdot(1 / 3)=1 / 3 . \quad \square
\end{aligned}
$$


The following observation connects the correlation between a function and a class of protocols with its discrepancy (see Section 2.1).

Fact 4.7. For every function $F_{k^{\prime}, N}:\left(\{0,1\}^{N}\right)^{k^{\prime}} \rightarrow\{-1,+1\}$, for every distribution $\mu$ on $\left(\{0,1\}^{N}\right)^{k^{\prime}}$, and for every $c$,

$$
\operatorname{corr}_{\mu}\left(F_{k^{\prime}, N}, \Pi_{k^{\prime}, N}^{c}\right) \leq 2^{c} \cdot \operatorname{disc}_{\mathrm{NOF}, \mu}\left(F_{k^{\prime}, N}\right) .
$$

Proof of Fact 4.7. Let $\pi \in \Pi_{k^{\prime}, N}^{c}$ be the protocol achieving the highest correlation with $F_{k^{\prime}, N}$. So, $\operatorname{corr}_{\mu}\left(F_{k^{\prime}, N}, \pi\right)=\operatorname{corr}_{\mu}\left(F_{k^{\prime}, N}, \Pi_{k^{\prime}, N}^{c}\right)$. By Fact 2.2, there exists a partition of $\left(\{0,1\}^{N}\right)^{k^{\prime}}$ into at most $2^{c} \pi$-monochromatic cylinder intersections $T_{1}, \ldots, T_{2^{c}}$. Let

$$
\operatorname{err}\left(T_{i}\right):=\operatorname{Pr}_{x \sim \mu}\left[F_{k^{\prime}, N}(x) \neq \pi(x) \wedge x \in T_{i}\right] .
$$

Observe that $\operatorname{err}\left(T_{i}\right)$ is the contribution of $T_{i}$ to the total error in $\pi$. That is,

$$
\operatorname{Pr}_{x \sim \mu}\left[F_{k^{\prime}, N}(x) \neq \pi(x)\right]=\sum_{i=1}^{2^{c}} \operatorname{err}\left(T_{i}\right) .
$$

Moreover,

$$
\operatorname{err}\left(T_{i}\right) \geq \frac{\mu\left(T_{i}\right)-\operatorname{disc}_{\mu}\left(F_{k^{\prime}, N}, T_{i}\right)}{2} \geq \frac{\mu\left(T_{i}\right)-\operatorname{disc}_{\mathrm{NOF}, \mu}\left(F_{k^{\prime}, N}\right)}{2} .
$$

Summing over all $T_{i}$,

$$
\operatorname{Pr}_{x \sim \mu}\left[F_{k^{\prime}, N}(x) \neq \pi(x)\right] \geq \frac{1-2^{c} \cdot \operatorname{disc}_{\mathrm{NOF}, \mu}\left(F_{k^{\prime}, n}\right)}{2} .
$$

Hence, using Fact 4.3 ,

$$
\begin{aligned}
\operatorname{corr}_{\mu}\left(F_{k^{\prime}, N}, \Pi_{k^{\prime}, N}^{c}\right) & =\operatorname{corr}_{\mu}\left(F_{k^{\prime}, N}, \pi\right) \\
& =1-2 \cdot \operatorname{Pr}_{x \sim \mu}\left[F_{k^{\prime}, N}(x) \neq \pi(x)\right] \leq 2^{c} \cdot \operatorname{disc}_{\mathrm{NOF}, \mu}\left(F_{k^{\prime}, n}\right)
\end{aligned}
$$

\subsubsection{Approximate Degree}

For a function $f_{m}:\{0,1\}^{m} \rightarrow \mathbb{R}$, the $\epsilon$-approximate degree of $f$ is the smallest $d=d(m)$ for which there exists a multivariate polynomial $g: \mathbb{R}^{m} \rightarrow \mathbb{R}$ of degree $d$ such that 
$\max _{x}|f(x)-g(x)| \leq \epsilon$. We use the following result of [NS94]; see [Pat92] for a result that applies to more functions.

Fact 4.8 ([NS94]). There exists a constant $\gamma>0$ such that for large enough $m$, the (5/6)approximate degree of the Or function on $m$ bits is at least $\gamma \cdot \sqrt{m}$.

The following key result shows that if a function $f$ has $\epsilon$-approximate degree $d$ then there is another function $g$ and a distribution $\mu$ such that, under $\mu, g$ is orthogonal to degree- $d$ polynomials and it has correlation $\epsilon$ with $f$. [She08b] gives references in the mathematics literature and points out a short proof by duality.

Fact 4.9 ([She08b]). Let $f:\{0,1\}^{m} \rightarrow\{-1,+1\}$ be a function with $\epsilon$-approximate degree $d$. There exist a function $g:\{0,1\}^{m} \rightarrow\{-1,+1\}$ and a distribution $\mu$ on $\{0,1\}^{m}$ such that:

(i) $\operatorname{corr}_{\mu}(g, f) \geq \epsilon$; and

(ii) for every $T \subseteq[m]$ with $|T| \leq d$ and every function $h:\{0,1\}^{|T|} \rightarrow \mathbb{R}$, we have $\mathrm{E}_{x \sim \mu}[g(x) \cdot h(x \mid T)]=0$,

where $x \mid T$ denotes the $m$ bits of $x$ indexed by $T$.

\subsection{Non-explicit Separation}

In this section we prove the following non-explicit separation between nondeterministic and randomized protocols.

Theorem 4.10. For every $\delta<1$ and for every function $k^{\prime}=k^{\prime}(N)$ satisfying $k^{\prime} \leq \delta \cdot \log N$ for all sufficiently large $N$, there exist constants $\epsilon, \alpha>0$ such that, for $m:=N^{\epsilon}$ and for all sufficiently large $N$, the following holds. There is a distribution $\lambda$ on $\left(\{0,1\}^{N}\right)^{k^{\prime}}$ such that if we choose a selection function $\phi_{k, N}:\left(\{0,1\}^{N}\right)^{k} \rightarrow\left(\begin{array}{c}{[N]} \\ m\end{array}\right)$ uniformly at random, we have:

$$
\underset{\phi_{k, N}}{\mathrm{E}}\left[\operatorname{corr}_{\lambda}\left(\operatorname{Lift}\left(\mathrm{OR}_{m}, \phi_{k, N}\right), \Pi_{k+1, N}^{N^{\alpha}}\right)\right]<1 / 3
$$


We obtain our lower bound on the randomized communication complexity of Lift $(\mathrm{OR}, \phi)$ using an analysis that follows [CA08]. In their paper, [CA08] analyzed the Set Intersection function, and for that reason, their selection function $\phi$ must be the And function. In our case, we allow $\phi$ to be a random function. While our results no longer apply to the Set Intersection function, we still obtain a separation between randomized and nondeterministic communication $\left(\mathrm{BPP}_{k}^{\mathrm{cc}}\right.$ and $\mathrm{NP}_{k}^{\mathrm{cc}}$ ) because, no matter what selection function is used, $\operatorname{Lift}(\mathrm{OR}, \phi)$ always has an efficient nondeterministic protocol.

At a more technical level, the results of [CA08] require $k<\log \log N$ because of the relationship between $N$ (the size of the input of player 0 ) and $m$ (the number of bits the base function OR gets applied to). For their analysis to go through, they need $N>2^{2^{k}} \cdot m^{O(1)}$. In our case, $N=2^{k} \cdot m^{O(1)}$ is sufficient, and this allows our results to be non-trivial for $k \leq \delta \cdot \log N$ for any $\delta<1$.

As mentioned earlier, we start with the base function OR : $\{0,1\}^{m} \rightarrow\{-1,+1\}$, for $m=N^{\epsilon} \ll N$. We lift the base function OR in order to obtain the lifted function Lift $(\mathrm{OR}, \phi)$. Our goal is to prove that, for a random $\phi, \operatorname{Lift}(\mathrm{OR}, \phi)$ has high randomized communication complexity.

A result of [NS94] gave a lower bound for the approximate degree of the Or function. By Fact 4.9, this implies that there exists a function $g:\{0,1\}^{m} \rightarrow\{-1,+1\}$ and a distribution $\mu$ on $\{0,1\}^{m}$ such that the functions $g$ and OR are highly correlated over $\mu$ and, furthermore, $g$ is orthogonal to low-degree polynomials. Now, we lift the function $g$ to obtain $\operatorname{Lift}(g, \phi)$. We also define $\lambda$ to be a distribution on $\left(\{0,1\}^{N}\right)^{k+1}$ where the $y_{i}{ }^{\prime}$ s are chosen uniformly at random and $x$ is also chosen uniformly at random, except on the $m$ bits indexed by $\phi\left(y_{1}, \ldots, y_{k}\right)$ which are selected according to $\mu$. Since $g$ and OR are highly correlated with respect to $\mu$, it is not hard to see that the lifted functions $\operatorname{Lift}(\mathrm{OR}, \phi)$ and $\operatorname{Lift}(g, \phi)$ are also highly correlated with respect to $\lambda$. Therefore, to prove that Lift $(\mathrm{OR}, \phi)$ has low correlation with $c$-bit protocols it suffices 
to prove that Lift $(g, \phi)$ has. For this, we use the original discrepancy method [BNS92]. As explained in Fact 2.8, this involves bounding the average value of Lift $(g, \phi)$ on certain $k$-dimensional cubes. To this end, we need to analyze the distribution of the $2^{k}$ sets that arise from evaluating $\phi$ on the $2^{k}$ points of the cube. Specifically, we are interested in how much these $2^{k}$ sets are "spread out," as measured by the size of their union. If the sets are spread out, we use (in Lemma 4.15) the fact that $g$ is orthogonal to low-degree polynomials to bound the average value of $\operatorname{Lift}(g, \phi)$ on the cubes. This step is similar to [She09, Cha07, LS08, CA08]. The main novelty in our analysis is that since we choose $\phi$ at random, we can prove good lower bounds (in Lemma 4.17) on the probability that the sets are indeed spread out.

Proof of Theorem 4.10 Let $\epsilon:=(1-\delta) / 5$ and let $\alpha:=\epsilon / 4$. Let $m:=N^{\epsilon}$ and let $c:=N^{\alpha}$. We write OR for $\mathrm{OR}_{m}, \phi$ for $\phi_{k, N}$, and $\Pi^{c}$ for $\Pi_{k+1, N}^{c}$.

Let $N$ be large enough so that, by Fact 4.8, the 5/6-approximate degree of OR is $\gamma \cdot \sqrt{m}$. Combining Facts 4.8 and 4.9 , we see that there exists a function $g:\{0,1\}^{m} \rightarrow$ $\{-1,+1\}$ and a distribution $\mu$ on $\{0,1\}^{m}$ such that:

(i) $\operatorname{corr}_{\mu}(g, \mathrm{OR}) \geq 5 / 6$; and

(ii) for every $T \subseteq[m],|T| \leq \gamma \sqrt{m}$ and $h:\{0,1\}^{|T|} \rightarrow \mathbb{R}, \mathrm{E}_{x \sim \mu}[g(x) h(x \mid T)]=0$.

Define the distribution $\lambda$ on $\left(\{0,1\}^{N}\right)^{k+1}$ as follows. For $x, y_{1}, \ldots, y_{k} \in\{0,1\}^{N}$, let

$$
\lambda\left(x, y_{1}, \ldots, y_{k}\right):=\frac{\mu\left(x \mid \phi\left(y_{1}, \ldots, y_{k}\right)\right)}{2^{(k+1) N-m}} .
$$

That is, we select $y_{1}, \ldots, y_{k}$ uniformly at random and then we select the bits of $x$ indexed by $\phi\left(y_{1}, \ldots, y_{k}\right)$ according to $\mu$ and the others uniformly.

Claim 4.11. $\operatorname{corr}_{\lambda}(\operatorname{Lift}(g, \phi), \operatorname{Lift}(\mathrm{OR}, \phi))=\operatorname{corr}_{\mu}(g, \mathrm{OR})$. 
Proof of Claim 4.11. Let $x^{\prime}=x \mid \phi\left(y_{1}, \ldots, y_{k}\right)$ and $x^{\prime \prime}=x \mid\left([N] \backslash \phi\left(y_{1}, \ldots, y_{k}\right)\right)$. Then,

$$
\begin{aligned}
\operatorname{corr}_{\lambda}(\operatorname{Lift}(g, \phi), \operatorname{Lift}(\mathrm{OR}, \phi)) & =\underset{\left(x, y_{1}, \ldots, y_{k}\right) \sim \lambda}{\mathrm{E}}\left[(\operatorname{Lift}(g, \phi) \cdot \operatorname{Lift}(\mathrm{OR}, \phi))\left(x, y_{1}, \ldots, y_{k}\right)\right] \\
& =\sum_{y_{1}, \ldots, y_{k}} \sum_{x^{\prime \prime}} \sum_{x^{\prime}} \frac{1}{2^{(k+1) N-m}} \cdot(\mu \cdot g \cdot \mathrm{OR})\left(x^{\prime}\right) \\
& =\sum_{x^{\prime}}(\mu \cdot g \cdot \mathrm{OR})\left(x^{\prime}\right)=\operatorname{corr}_{\mu}(g, \mathrm{OR}),
\end{aligned}
$$

where in the second to last step we use the fact that there are a total of $2^{(k+1) N-m}$ terms in the outer sums.

Claim 4.12. $\operatorname{corr}_{\lambda}\left(\operatorname{Lift}(\mathrm{OR}, \phi), \Pi^{c}\right) \leq \operatorname{corr}_{\lambda}\left(\operatorname{Lift}(g, \phi), \Pi^{c}\right)+1 / 6$.

Proof of Claim 4.12. Let $\pi \in \Pi^{c}$ be the protocol that has the highest correlation with Lift $(\mathrm{OR}, \phi)$ under $\lambda$. Using Claims 4.11 and 4.4 and Property (i) of $g$, we get

$$
\begin{aligned}
\operatorname{corr}_{\lambda}\left(\operatorname{Lift}(\mathrm{OR}, \phi), \Pi^{c}\right) & =\operatorname{corr}_{\lambda}(\operatorname{Lift}(\mathrm{OR}, \phi), \pi) \\
& \leq \operatorname{corr}_{\lambda}(\operatorname{Lift}(g, \phi), \pi)+1-\operatorname{corr}_{\lambda}(\operatorname{Lift}(g, \phi), \operatorname{Lift}(\mathrm{OR}, \phi)) \\
& =\operatorname{corr}_{\lambda}(\operatorname{Lift}(g, \phi), \pi)+1-\operatorname{corr}_{\mu}(g, \mathrm{OR}) \\
& \leq \operatorname{corr}_{\lambda}(\operatorname{Lift}(g, \phi), \pi)+1 / 6 \\
& \leq \operatorname{corr}_{\lambda}\left(\operatorname{Lift}(g, \phi), \Pi^{c}\right)+1 / 6
\end{aligned}
$$

Thus, we only have to bound $\operatorname{corr}_{\lambda}\left(\operatorname{Lift}(g, \phi), \Pi^{c}\right)$, and this is addressed next.

\section{Claim 4.13.}

$$
\left(\operatorname{corr}_{\lambda}\left(\operatorname{Lift}(g, \phi), \Pi^{c}\right)\right)^{2^{k}} \leq 2^{(c+m) 2^{k}} \cdot \underset{\bar{y}^{0}, \bar{y}^{1}}{\mathrm{E}}\left[\left|\underset{x}{\mathrm{E}}\left[\prod_{u \in\{0,1\}^{k}}(\mu \cdot g)\left(x \mid \phi\left(\bar{y}^{u}\right)\right)\right]\right|\right] .
$$

Proof of Claim 4.13 .

$$
\begin{aligned}
\operatorname{corr}_{\lambda}\left(\operatorname{Lift}(g, \phi), \Pi^{c}\right) & \leq 2^{c} \cdot \operatorname{disc}_{\mathrm{NOF}, \lambda}(\operatorname{Lift}(g, \phi)) \\
& =2^{c} \cdot\left|\underset{(x, \bar{y}) \sim \lambda}{\mathrm{E}}\left[\left(\operatorname{Lift}(g, \phi) \cdot 1_{T}\right)(x, \bar{y})\right]\right|,
\end{aligned}
$$$$
\text { (by Fact 4.7) }
$$ 
where $T$ is the cylinder intersection witnessing the discrepancy of $\operatorname{Lift}(g, \phi)$ under $\lambda$,

$$
\begin{aligned}
& =2^{c} \cdot\left|\sum_{x, \bar{y}}\left(\lambda \cdot \operatorname{Lift}(g, \phi) \cdot 1_{T}\right)(x, \bar{y})\right| \\
& =2^{c} \cdot\left|\sum_{x, \bar{y}} \frac{(\mu \cdot g)(x \mid \phi(\bar{y}))}{2^{(k+1) \cdot N-m}} \cdot 1_{T}(x, \bar{y})\right|,
\end{aligned}
$$

where we have used the definitions of $\lambda$ and $\operatorname{Lift}(g, \phi)$,

$$
=2^{c+m} \cdot\left|\underset{x, \bar{y} \sim \mathcal{U}}{\mathrm{E}}\left[(\mu \cdot g)(x \mid \phi(\bar{y})) \cdot 1_{T}(x, \bar{y})\right]\right| .
$$

We are now in a position to apply the version of the [BNS92] argument given as Fact 2.9, obtaining:

$$
\left(\operatorname{corr}_{\lambda}\left(\operatorname{Lift}(g, \phi), \Pi^{c}\right)\right)^{2^{k}} \leq 2^{(c+m) 2^{k}} \underset{\bar{y}^{0}, \bar{y}^{1}}{\mathrm{E}}\left[\mid \underset{x}{\mathrm{E}}\left[\prod_{u \in\{0,1\}^{k}}(\mu \cdot g)(x \mid \phi(\bar{y}))\right]\right] .
$$

Henceforth, our analysis makes extensive use of the following notation.

Definition 4.14. Let $\mathcal{S}=\left(S_{1}, \ldots, S_{z}\right)$ be a multiset of $m$-element subsets of $[N]$. Let the range of $\mathcal{S}$, denoted by $\cup \mathcal{S}$, be the set of indices from $[N]$ that appear in at least one set in $\mathcal{S}$. Let the boundary of $\mathcal{S}$, denoted by $\partial \mathcal{S}$, be the set of indices from $[N]$ that appear in exactly one set in the collection $\mathcal{S}$.

For $\bar{y}^{0}=\left(y_{1}^{0}, \ldots, y_{k}^{0}\right), \bar{y}^{1}=\left(y_{1}^{1}, \ldots, y_{k}^{1}\right) \in\left(\{0,1\}^{N}\right)^{k}$, and for $u \in\{0,1\}^{k}$, define $S_{u}\left(\bar{y}^{0}, \bar{y}^{1}, \phi\right):=\phi\left(y_{1}^{u_{1}}, \ldots, y_{k}^{u_{k}}\right)$. Let $\mathcal{S}\left(\bar{y}^{0}, \bar{y}^{1}, \phi\right)$ be the multiset $\left(S_{u}: u \in\{0,1\}^{k}\right)$. We define the number of conflicts in $\mathcal{S}$ to be $q(\mathcal{S}):=m \cdot 2^{k}-|\cup \mathcal{S}|$. We write $S_{u}$ for $S_{u}\left(\bar{y}^{0}, \bar{y}^{1}, \phi\right)$ and $\mathcal{S}$ for $\mathcal{S}\left(\bar{y}^{0}, \bar{y}^{1}, \phi\right)$.

Intuitively, $|\cup \mathcal{S}|$ measures the range of $\mathcal{S}$, while $m \cdot 2^{k}$ is the maximum possible value for this range. We use the following three Lemmas to complete our proof. The first, Lemma 4.15, deals with the case where the multiset $\mathcal{S}$ has few conflicts. In this case, we argue that one of the sets $S_{u} \in \mathcal{S}$ has a very small intersection with the rest of the other sets, which allows us to apply Property (ii) of $g$ and $\mu$ to obtain the stated bound. A variant of Lemma 4.15 appears in [CA08]. 
Lemma 4.15. For every $\bar{y}^{0}, \bar{y}^{1}$ and $\phi$, if $q\left(\mathcal{S}\left(\bar{y}^{0}, \bar{y}^{1}, \phi\right)\right)<\gamma \cdot \sqrt{m} \cdot 2^{k} / 2$, then

$$
\underset{x}{\mathrm{E}}\left[\prod_{u \in\{0,1\}^{k}}(\mu \cdot g)\left(x \mid S_{u}\left(\bar{y}^{0}, \bar{y}^{1}, \phi\right)\right)\right]=0
$$

Lemma 4.16 gives a bound in terms of the number of conflicts in $\mathcal{S}$ which only uses the fact that $\mu$ is a probability distribution. A slightly weaker version of this lemma appeared originally in [CA08]. Independently of our work, Chattopadhyay and Ada have subsequently also derived the stronger statement we give below.

Lemma 4.16. For every $\bar{y}^{0}, \bar{y}^{1}$ and $\phi$ :

$$
\underset{x}{\mathrm{E}}\left[\prod_{u \in\{0,1\}^{k}} \mu\left(x \mid S_{u}\left(\bar{y}^{0}, \bar{y}^{1}, \phi\right)\right)\right] \leq \frac{2^{q\left(\mathcal{S}\left(\bar{y}^{0}, \bar{y}^{1}, \phi\right)\right)}}{2^{m \cdot 2^{k}}} .
$$

Lemma 4.17 is the key place where we exploit the fact that $\phi$ is chosen at random to obtain an upper bound on the probability of having a given number of conflicts in $\mathcal{S}$.

Lemma 4.17. For every $q>0$ and uniformly chosen $\bar{y}^{0}, \bar{y}^{1}, \phi$ :

$$
\operatorname{Pr}_{\bar{y}^{0}, \bar{y}^{1}, \phi}\left[q\left(\mathcal{S}\left(\bar{y}^{0}, \bar{y}^{1}, \phi\right)\right)=q\right] \leq\left(\frac{m^{3} \cdot 2^{2 k}}{q \cdot N}\right)^{q} .
$$

Before proving these Lemmas, we complete the proof of our main theorem. We 
have the following derivation. For a uniformly chosen $\phi$ :

$\underset{\phi}{\mathrm{E}}\left[\operatorname{corr}_{\lambda}\left(\operatorname{Lift}(g, \phi), \Pi^{c}\right)\right]^{2^{k}} \leq \underset{\phi}{\mathrm{E}}\left[\operatorname{corr}_{\lambda}\left(\operatorname{Lift}(g, \phi), \Pi^{c}\right)^{2^{k}}\right]$

$\leq 2^{(c+m) 2^{k}} \underset{\bar{y}^{0}, \bar{y}^{1}, \phi}{\mathrm{E}}\left[\left|\underset{x}{\mathrm{E}}\left[\prod_{u \in\{0,1\}^{k}}(\mu \cdot g)\left(x \mid S_{u}\right)\right]\right|\right] \quad$ (by Claim 4.13)

$=2^{(c+m) 2^{k}} \sum_{q \geq 0} \operatorname{Pr}_{\bar{y}^{0}, \tilde{y}^{1}, \phi}[q(\mathcal{S})=q] \cdot \underset{\bar{y}^{0}, \tilde{y}^{1}, \phi}{\mathrm{E}}\left[\left|\underset{x}{\mathrm{E}}\left[\prod_{u \in\{0,1\}^{k}}(\mu \cdot g)\left(x \mid S_{u}\right)\right]\right| \mid q(\mathcal{S})=q\right]$

$\leq 2^{(c+m) 2^{k}} \sum_{q \geq \frac{\gamma \sqrt{\bar{m} 2^{k}}}{2}} \operatorname{Pr}_{\bar{y}^{0}, \bar{y}^{1}, \phi}[q(\mathcal{S})=q] \cdot \operatorname{F}_{\bar{y}^{0}, \bar{y}^{1}, \phi}^{\mathrm{E}}\left[\left|\operatorname{E}_{x}\left[\prod_{u \in\{0,1\}^{k}}(\mu \cdot g)\left(x \mid S_{u}\right)\right]\right| \mid q(\mathcal{S})=q\right]$

(by Lemma 4.15)

$\leq 2^{(c+m) 2^{k}} \sum_{q \geq \frac{\gamma \sqrt{m} 2^{k}}{2}} \operatorname{Pr}_{\bar{y}^{0}, \bar{y}^{1}, \phi}[q(\mathcal{S})=q] \cdot \underset{\bar{y}^{0}, \tilde{y}^{1}, \phi}{\mathrm{E}}\left[\left|\underset{x}{\mathrm{E}}\left[\prod_{u \in\{0,1\}^{k}} \mu\left(x \mid S_{u}\right)\right]\right| \mid q(\mathcal{S})=q\right]$

(because $|g|=1$ )

$\leq 2^{(c+m) 2^{k}} \sum_{q \geq \frac{\gamma \sqrt{m} 2^{k}}{2}} \operatorname{Pr}_{\bar{y}^{0}, \bar{y}^{1}, \phi}[q(\mathcal{S})=q] \cdot \frac{2^{q}}{2^{m 2^{k}}}=2^{c 2^{k}} \sum_{q \geq \frac{\gamma \sqrt{m} 2^{k}}{2}} \operatorname{Pr}_{\bar{y}^{3}, \bar{y}^{1}, \phi}[q(\mathcal{S})=q] \cdot 2^{q}$

(by Lemma 4.16)

$\leq 2^{c 2^{k}} \sum_{q \geq \frac{\gamma \sqrt{m} 2^{k}}{2}}\left(\frac{m^{3} \cdot 2^{2 k}}{q \cdot N}\right)^{q} \cdot 2^{q} \quad$ (by Lemma 4.17)

$=2^{c 2^{k}} \sum_{q \geq \frac{\gamma \sqrt{m} 2^{k}}{2}}\left(\frac{2 \cdot m^{3} \cdot 2^{2 k}}{q \cdot N}\right)^{q} \leq 2^{c 2^{k}} \sum_{q \geq \frac{\gamma \sqrt{m} 2^{k}}{2}}\left(\frac{4 \cdot m^{3} \cdot 2^{2 k}}{\gamma \cdot \sqrt{m} \cdot 2^{k} \cdot N}\right)^{q} \quad\left(\right.$ using $\left.q \geq \frac{\gamma \cdot \sqrt{m} \cdot 2^{k}}{2}\right)$

$\leq 2^{c 2^{k}} \sum_{q \geq \gamma \sqrt{m} 2^{k} / 2}\left(\frac{4}{\gamma} \cdot N^{\frac{5 \epsilon}{2}-(1-\delta)}\right)^{q} \quad\left(\right.$ using $m=N^{\epsilon}$, and $\left.k \leq \delta \cdot \log N\right)$

$\leq 2^{c 2^{k}} \sum_{q \geq \frac{\gamma \sqrt{m} 2^{k}}{2}}\left(\frac{1}{2}\right)^{q} \quad\left(\right.$ using $\epsilon=\frac{1-\delta}{5}$, for large enough $\left.N\right)$

$\leq 2^{c \cdot 2^{k}+1-\frac{\gamma \sqrt{m} 2^{k}}{2}} \leq 2^{2^{k}\left(N^{\alpha}-(\gamma / 2) \cdot N^{\epsilon / 2}\right)}$

Since $\alpha=\epsilon / 4$, for sufficiently large $N, \mathrm{E}_{\phi}\left[\operatorname{corr}_{\lambda}\left(\operatorname{Lift}(g, \phi), \Pi^{N^{\alpha}}\right)\right]<1 / 6$. Combining 
this with Claim 4.12 we complete the proof of Theorem 4.10 :

$$
\underset{\phi}{\mathrm{E}}\left[\operatorname{corr}_{\lambda}\left(\operatorname{Lift}(\mathrm{OR}, \phi), \Pi^{N^{\alpha}}\right)\right]<1 / 6+1 / 6=1 / 3 \text {. }
$$

It is left to prove the Lemmas. For this, the reader may want to recall Definition 4.14.

Proof of Lemma 4.15, Let $r(\mathcal{S})=|\bigcup \mathcal{S}|$ be the size of the range of $\mathcal{S}$, and let $b(\mathcal{S})=|\partial \mathcal{S}|$ be the size of the boundary of $\mathcal{S}$. Note that $r(\mathcal{S})-b(\mathcal{S}) \leq q(\mathcal{S})$ because every $j \in$ $\cup \mathcal{S} \backslash \partial \mathcal{S}$ occurs in at least 2 sets in $\mathcal{S}$, thus contributes at least 1 to $q(\mathcal{S})$. Furthermore, $r(\mathcal{S})+q(\mathcal{S})=m \cdot 2^{k}$. Then, $\sum_{u \in\{0,1\}^{k}}\left|S_{u} \cap \partial \mathcal{S}\right|=b(\mathcal{S}) \geq r(\mathcal{S})-q(\mathcal{S})=m \cdot 2^{k}-2$. $q(\mathcal{S})>(m-\gamma \sqrt{m}) 2^{k}$. By the pigeonhole principle, there exists $v$ such that $\left|S_{v} \cap \partial \mathcal{S}\right|>$ $m-\gamma \sqrt{m}$. We can write

$$
\underset{x}{\mathrm{E}}\left[\prod_{u \in\{0,1\}^{k}}(\mu \cdot g)\left(x \mid S_{u}\right)\right]=\underset{x \mid S_{v}}{\mathrm{E}}\left[(\mu \cdot g)\left(x \mid S_{v}\right) \underset{x \mid[N] \backslash S_{v}}{\mathrm{E}}\left[\prod_{\substack{u \in\{0,1\}^{k} \\ u \neq v}}(\mu \cdot g)\left(x \mid S_{u}\right)\right]\right],
$$

where $x \sim \mathcal{U}\left(\{0,1\}^{N}\right), x \mid S_{v} \sim \mathcal{U}\left(\{0,1\}^{m}\right)$ and $x \mid[N] \backslash S_{v} \sim \mathcal{U}\left(\{0,1\}^{N-m}\right)$.

Let $T=S_{v} \backslash \partial \mathcal{S}$. So $|T| \leq \gamma \sqrt{m}$. Let $h=\mathrm{E}_{x \mid[N] \backslash S_{v}}\left[\prod_{u \neq v}(\mu \cdot g)\left(x \mid S_{u}\right)\right]$. Since $h$ depends only on $x \mid T$, by Property (ii) of $g$ and $\mu, \mathrm{E}_{x \mid S_{v}}\left[(\mu \cdot g)\left(x \mid S_{v}\right) \cdot h(x \mid T)\right]=0$.

Proof of Lemma 4.16. We see that

$$
\underset{x}{\mathrm{E}}\left[\prod_{u \in\{0,1\}^{k}} \mu\left(x \mid S_{u}\right)\right]=\underset{x \mid \cup \mathcal{S}}{\mathrm{E}}\left[\prod_{u \in\{0,1\}^{k}} \mu\left(x \mid S_{u}\right)\right],
$$

as each $\mu\left(x \mid S_{u}\right)$ only depends on the bits of $x$ in $\cup \mathcal{S}$. Identifying $u \in\{0,1\}^{k}$ with $u \in\left[2^{k}\right]=\left\{1, \ldots, 2^{k}\right\}$, let $\mathcal{S}_{u}$ be the sub-multiset of $\mathcal{S}$ consisting of the sets up to and including $S_{u}: \mathcal{S}_{u}:=\left(S_{1}, \ldots, S_{u}\right)$. So, $\mathcal{S}=\mathcal{S}_{2^{k}}$. Also define $\mathcal{S}_{0}:=\varnothing$. Furthermore, let $G_{u}:=\mathrm{E}_{x \mid \cup \mathcal{S}_{u}}\left[\prod_{i=1}^{u} \mu\left(x \mid S_{i}\right)\right]$ and let $H_{u}\left(x \mid S_{u} \backslash \partial \mathcal{S}_{u}\right):=\mathrm{E}_{x \mid S_{u} \cap \partial \mathcal{S}_{u}}\left[\mu\left(x \mid S_{u}\right)\right]$. Note that this quantity depends on the bits of $x$ in $S_{u} \backslash \partial \mathcal{S}_{u}$, i.e. on $x \mid S_{u} \backslash \partial \mathcal{S}_{u}$. Letting $G_{0}:=1$, observe that, for $1 \leq u \leq 2^{k}$,

$$
G_{u}=\underset{x \mid \cup \mathcal{S}_{u-1}}{\mathrm{E}}\left[\left(\prod_{i=1}^{u-1} \mu\left(x \mid S_{i}\right)\right) \cdot H_{u}\left(x \mid S_{u} \backslash \partial \mathcal{S}_{u}\right)\right] \leq G_{u-1} \cdot \max _{x \mid\left(S_{u} \backslash \partial \mathcal{S}_{u}\right)}\left(H_{u}\right) .
$$


To obtain a bound on $\max \left(H_{u}\right)$, consider an arbitrary partition of $[m]$ into two sets $E, F$. Let $v$ be a distribution on $[m]$, and let $\rho(x \mid E)=\mathrm{E}_{x \mid F}[v(x)]$. Then, $\rho(x \mid E)=$ $\sum_{x \mid F} 2^{-|F|} \cdot v(x)=2^{-|F|} \sum_{x \mid F} v(x) \leq 2^{-|F|}=2^{|E|-m}$, simply using the fact that $v$ is a probability distribution. Thus, $\max _{x \mid\left(S_{u} \backslash \partial \mathcal{S}_{u}\right)}\left(H_{u}\right) \leq 2^{\left|S_{u} \backslash \partial \mathcal{S}_{u}\right|-m}$. Inductively,

$$
\underset{x}{\mathrm{E}}\left[\prod_{u=1}^{2^{k}} \mu\left(x \mid S_{u}\right)\right]=G_{2^{k}} \leq \frac{2^{\sum_{u=1}^{2^{k}}\left|S_{u} \backslash \partial \mathcal{S}_{u}\right|}}{2^{m \cdot 2^{k}}} .
$$

Consider some index $z \in \cup \mathcal{S}$. Suppose this index appears in $l$ sets $S_{u_{1}}, \ldots, S_{u_{l}}$ from $\mathcal{S}$, with $u_{1}<\cdots<u_{l}$. Then, this index contributes exactly $l-1$ to the expression $\sum_{u=1}^{2^{k}}\left|S_{u} \backslash \partial \mathcal{S}_{u}\right|$, once for every $u=u_{2}, \ldots, u_{l}$ (for $u=u_{1}, z \in \partial \mathcal{S}_{u}$ because no set before $S_{u}$ contains $z$ ). Since this holds for every index $z$, we see that $\sum_{u=1}^{2^{k}}\left|S_{u} \backslash \partial \mathcal{S}_{u}\right|=q(\mathcal{S})$ and therefore $\mathrm{E}_{x}\left[\prod_{u \in\{0,1\}^{k}} \mu\left(x \mid S_{u}\right)\right] \leq 2^{q(\mathcal{S})-m 2^{k}}$.

For reasons which we explain in Section 4.3, we split the proof of Lemma 4.17 in two parts. We first prove the following fact, which we reuse later on.

Lemma 4.18. Let $\mathcal{S}=\left(S_{u} \mid u \in\{0,1\}^{k}\right)$ be a collection of $2^{k}$ m-element subsets of $[N]$. When these sets are picked independently and uniformly at random, for every $q>0$,

$$
\operatorname{Pr}_{\mathcal{S} \sim \mathcal{U}\left(\left(\begin{array}{c}
\left.[N]^{2}\right)^{k} \\
m
\end{array}\right)\right.}[q(\mathcal{S})=q] \leq\left(\frac{e \cdot m^{2} \cdot 2^{2 k}}{q \cdot N}\right)^{q} .
$$

Proof of Lemma 4.18. We identify $\{0,1\}^{k}$ with $\left[2^{k}\right]=\left\{1, \ldots, 2^{k}\right\}$. For $1 \leq i \leq 2^{k}$, let $\mathcal{S}_{i}=\left(S_{1}, \ldots, S_{i}\right)$. So, $\mathcal{S}=\mathcal{S}_{2^{k}}$. Let $\mathcal{S}_{0}=\varnothing$. Let $Q=q(\mathcal{S})$. For $1 \leq i \leq 2^{k}$, let $Q_{i}$ be the number of conflicts obtained while picking $S_{i}$ after having picked $\mathcal{S}_{i-1}$ and let $R_{i}$ be the range of $\mathcal{S}_{i}$. Formally, $Q_{i}=\left|S_{i} \cap\left(\bigcup \mathcal{S}_{i-1}\right)\right|$ and $R_{i}=\left|\cup \mathcal{S}_{i}\right|$. It is easy to see that $Q=\sum_{i=1}^{2^{k}} Q_{i}$. Then,

$$
\begin{aligned}
\operatorname{Pr}[Q=q] & =\sum_{\substack{q_{1}, \ldots, q_{2^{k}}: \\
\sum q_{i}=q}} \operatorname{Pr}\left[\forall i, Q_{i}=q_{i}\right] \\
& =\sum_{\substack{q_{1}, \ldots, q_{2^{k}}: \\
\sum q_{i}=q}} \prod_{i=1}^{2^{k}} \operatorname{Pr}\left[Q_{i}=q_{i} \mid \forall j<i, Q_{j}=q_{j}\right]
\end{aligned}
$$


By the nature of the experiment, the probability of obtaining $q_{i}$ conflicts while picking $S_{i}$ depends only on the range of the sets picked before, thus

$$
\operatorname{Pr}\left[Q_{i}=q_{i} \mid \forall j<i, Q_{j}=q_{j}\right]=\operatorname{Pr}\left[Q_{i}=q_{i} \mid R_{i-1}=(i-1) m-\sum_{1 \leq j<i} q_{j}\right] .
$$

For $m+r \leq N$ and $0 \leq q^{\prime} \leq m$, let $C\left(N, m, q^{\prime}, r\right)$ denote the probability that, when picking an $m$-element subset of $[N]$ we obtain exactly $q^{\prime}$ conflicts, conditioned on the fact that the range of elements picked so far is exactly $r$. We have

$$
\begin{aligned}
C\left(N, m, q^{\prime}, r\right) & =\left(\begin{array}{l}
m \\
q^{\prime}
\end{array}\right) \frac{N-r}{N} \cdot \frac{N-r-1}{N} \cdot \ldots \cdot \frac{N-r-\left(m-q^{\prime}-1\right)}{N} \cdot\left(\frac{r+m-q^{\prime}}{N}\right)^{q^{\prime}} \\
& \leq\left(\begin{array}{c}
m \\
q^{\prime}
\end{array}\right)\left(\frac{r+m}{N}\right)^{q^{\prime}}
\end{aligned}
$$

Since $m \cdot 2^{k} \leq N^{\epsilon+\delta}, \epsilon+\delta<1$, and $i \leq 2^{k}$,

$$
\begin{aligned}
\operatorname{Pr}\left[Q_{i}=q_{i} \mid R_{i-1}=(i-1) m-\sum_{1 \leq j<i} q_{j}\right] & =C\left(N, m, q_{i},(i-1) m-\sum_{1 \leq j<i} q_{j}\right) \\
& =\left(\begin{array}{c}
m \\
q_{i}
\end{array}\right)\left(\frac{(i-1) m-\sum_{1 \leq j<i} q_{j}+m}{N}\right)^{q_{i}} \\
& \leq\left(\begin{array}{c}
m \\
q_{i}
\end{array}\right)\left(\frac{m \cdot 2^{k}}{N}\right)^{q_{i}} .
\end{aligned}
$$

Putting these together,

$$
\begin{aligned}
\operatorname{Pr}[Q=q] & \leq \sum_{\substack{q_{1}, \ldots, q_{2^{k}}: \\
\sum q_{i}=q}} \prod_{i=1}^{2^{k}}\left(\begin{array}{c}
m \\
q_{i}
\end{array}\right)\left(\frac{m \cdot 2^{k}}{N}\right)^{q_{i}} \\
& =\left(\frac{m \cdot 2^{k}}{N}\right)^{q}\left(\sum_{\substack{q_{1}, \ldots, q_{2^{k}}: \\
\sum q_{i}=q}}\left(\begin{array}{c}
m \\
q_{1}
\end{array}\right) \cdots \cdot\left(\begin{array}{c}
m \\
q_{2^{k}}
\end{array}\right)\right) \\
& =\left(\frac{m \cdot 2^{k}}{N}\right)^{q} \cdot\left(\begin{array}{c}
m \cdot 2^{k} \\
q
\end{array}\right) \\
& \leq\left(\frac{e \cdot m^{2} \cdot 2^{2 k}}{q \cdot N}\right)^{q} .
\end{aligned}
$$


Proof of Lemma 4.17. The multiset $\mathcal{S}$ consists of the $2^{k}$ sets $S_{u}=\phi\left(y_{1}^{u_{1}}, \ldots, y_{k}^{u_{k}}\right)$ for $u \in$ $\{0,1\}^{k}$. The probability over the choice of the $y^{\prime}$ s that for some $i, y_{i}^{0}=y_{i}^{1}$, is at most $k / 2^{n}$. When this event does not occur, the $2^{k}$ points at which $\phi$ gets evaluated are all distinct. Since $\phi$ is chosen at random, the $2^{k}$ outputs of $\phi$ are $2^{k}$ uniformly and independently random $m$-element subsets of $[N]$. By Lemma 4.18 , we have an upper bound for the probability of having $q$ conflicts in this case. Thus,

$$
\operatorname{Pr}_{\bar{y}^{0},,^{1}, \phi}[q(\mathcal{S})=q] \leq \frac{k}{2^{N}}+\left(\frac{e \cdot m^{2} \cdot 2^{2 k}}{q \cdot N}\right)^{q}
$$

Furthermore,

$$
\begin{aligned}
\left(\frac{e \cdot m^{2} \cdot 2^{2 k}}{q \cdot N}\right)^{q} & \geq\left(\frac{1}{N^{2}}\right)^{q} \quad\left(\operatorname{using} q \leq m \cdot 2^{k} \leq N\right) \\
& =\left(\frac{1}{2}\right)^{2 \cdot q \cdot \log N} \\
& \geq\left(\frac{1}{2}\right)^{N-\log k} \quad\left(\text { using } q \leq m \cdot 2^{k} \leq N^{\epsilon+\delta} \text { and } \epsilon+\delta<1\right) \\
& =\frac{k}{2^{N}} .
\end{aligned}
$$

Thus,

$$
\begin{aligned}
& \operatorname{Pr}_{\bar{y}^{0}, \bar{y}^{1}, \phi}[q(\mathcal{S})=q] \leq \frac{k}{2^{N}}+\left(\frac{e \cdot m^{2} \cdot 2^{2 k}}{q \cdot N}\right)^{q} \\
& \leq 2 \cdot\left(\frac{e \cdot m^{2} \cdot 2^{2 k}}{q \cdot N}\right)^{q} \\
& \leq 2^{q} \cdot\left(\frac{e \cdot m^{2} \cdot 2^{2 k}}{q \cdot N}\right)^{q} \quad(\text { since } q \geq 1) \\
& =(2 \cdot e)^{q} \cdot\left(\frac{m^{2} \cdot 2^{2 k}}{q \cdot N}\right)^{q} \\
& \leq m^{q} \cdot\left(\frac{m^{2} \cdot 2^{2 k}}{q \cdot N}\right)^{q} \quad \text { (for large enough } m \text { ) } \\
& =\left(\frac{m^{3} \cdot 2^{2 k}}{q \cdot N}\right)^{q} \text {. }
\end{aligned}
$$




\subsection{Explicit Separation}

In this Section, we give an explicit function separating randomized from nondeterministic NOF protocols, thus proving Theorem 4.1. We proceed in two stages as follows. First, we prove a "derandomized" version of Theorem 4.10, where we exponentially reduce the amount of randomness required in the choice of the selection function. Second, we argue that by including in the input the random seed used to define the selection function, we obtain an explicit function that is still hard for randomized protocols but easy for nondeterministic ones.

In the proof of Theorem 4.10 , the only place where we use the fact that the selection function $\phi:\left(\{0,1\}^{N}\right)^{k} \rightarrow\left(\begin{array}{c}{[N]} \\ m\end{array}\right)$ is chosen at random is inside Lemma 4.18. More specifically, in Lemma 4.17 we choose $\bar{y}^{0}=\left(y_{1}^{0}, \ldots, y_{k}^{0}\right), \bar{y}^{1}=\left(y_{1}^{1}, \ldots, y_{k}^{1}\right) \in$ $\left(\{0,1\}^{N}\right)^{k}$, we condition on $y_{i}^{0} \neq y_{i}^{1}$ for every $i \in[k]$, we evaluate $\phi$ at the $2^{k}$ points $\left(\bar{y}^{u}=\left(y_{1}^{u_{1}}, \ldots, y_{k}^{u_{k}}\right)\right)_{u \in\{0,1\}^{k}}$ (which are now distinct because of the conditioning), we obtain the collection of sets $\mathcal{S}=\left(S_{u}=\phi\left(\bar{y}^{u}\right)\right)_{u \in\{0,1\}^{k}}$, and in Lemma 4.18 we give a bound for the probability of obtaining many conflicts (equivalently, a small range) in the collection $\mathcal{S}$.

Intuitively, we see that the only property we need from the distribution on $\phi$ is " $2^{k}$-wise independence": for every fixed, distinct, $2^{k}$ points $z_{1}, \ldots, z_{2^{k}}$, when choosing $\phi$ at random, we need $\left(\phi\left(z_{1}\right), \ldots, \phi\left(z_{2^{k}}\right)\right)$ to be jointly uniformly distributed, i.e., distributed according to $\mathcal{U}\left(\left(\begin{array}{c}{[\mathrm{N}]} \\ m\end{array}\right)^{2^{k}}\right)$. Furthermore, if we think of the selection function as a deterministic function $\phi^{\prime}$ that also takes as input an $r$-bit random seed $\rho$, we see that, currently, choosing $\phi$ uniformly at random is equivalent to feeding $\phi^{\prime}$ a total of $r=2^{k N} \cdot \log \left(\begin{array}{l}N \\ m\end{array}\right)$ random bits (since $\rho$ must specify an $m$-element subset of $[N]$ for every possible $k \cdot N$-bit input). As the first stage towards proving Theorem 4.1 , we use a result of [NN93] to replace our selection function with another function $\psi$ whose distribution is now "almost $2^{k}$-wise independent", but $\psi$ uses only $O\left(k \cdot N+2^{k}\right)=N^{O(1)}$ 
random bits.

Proof of Theorem 4.1 Let $D_{1}$ and $D_{2}$ be two distributions on the same set $X$. We say that $D_{1}$ and $D_{2}$ are $\beta$-close in statistical distance, written $\left|D_{1}-D_{2}\right| \leq \beta$, if for every event $E \subseteq X$

$$
\left|\operatorname{Pr}_{x \sim D_{1}}[x \in E]-\operatorname{Pr}_{x \sim D_{2}}[x \in E]\right| \leq \beta .
$$

Given a function $h: X \times Y \rightarrow Z$, a collection of $K$ points $x_{1}, \ldots, x_{K} \in X$, and a distribution $D$ on $Y$, we denote by $h\left(\left(x_{i}\right)_{i \in[K]}, D\right)$ the distribution over $Z^{K}$ of the $K$-tuple $\left(h\left(x_{i}, y\right)\right)_{i \in[K]}$ when $y$ is chosen from $D$.

[NN93] gave an explicit construction of $N$ boolean variables such that any $k$ of them are $\beta$-close to the uniform distribution on $\{0,1\}^{k}$, using $O(\log N+k+\log (1 / \beta))$ truly random bits. We rephrase this as follows.

Fact 4.19 ([NN93]). Given $M, K$, and $\beta$, there exist $R \leq O(M+K+\log (1 / \beta))$ and $a$ polynomial time computable function $h:\{0,1\}^{M} \times\{0,1\}^{R} \rightarrow\{0,1\}$ such that for every fixed, distinct, $p_{1}, \ldots, p_{K} \in\{0,1\}^{M}$, we have

$$
\left|h\left(\left(p_{i}\right)_{i \in[K]}, \mathcal{U}\left(\{0,1\}^{R}\right)\right)-\mathcal{U}\left(\{0,1\}^{K}\right)\right| \leq \beta .
$$

We construct a new selection function $\psi_{k, N}:\left(\{0,1\}^{N}\right)^{k} \times\{0,1\}^{r} \rightarrow\left(\begin{array}{c}{[N]} \\ m\end{array}\right)$ as fol-

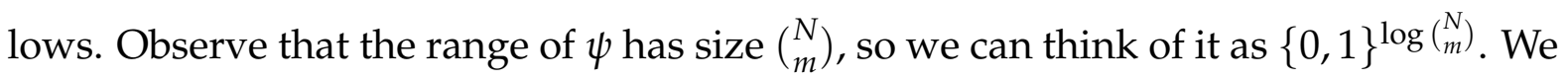
want $\psi$ to be uniform on this range. To achieve this, we use an extra $s \simeq \log \log \left(\begin{array}{l}N \\ m\end{array}\right)$ bits in the input to $h$, and we generate the $\log \left(\begin{array}{l}N \\ m\end{array}\right)$ bits of the output of $\psi_{k, N}$ by ranging over all settings of these extra input bits to $h$. We want $2^{k}$ evaluations of $\psi_{k, N}$ to be almost uniformly distributed, and, for each of those, we use $2^{s}$ evaluations of the boolean $h$. Thus, we need "almost $\left(K:=2^{k} \cdot 2^{s}\right)$-wise independence".

Definition 4.20. Let $s:=\left[\log \log \left(\left(\begin{array}{c}N \\ m\end{array}\right)^{6}\right)\right]$. Set $M:=k \cdot N+s, K:=2^{k} \cdot 2^{s}$ and $\beta:=$ $1 / N^{2 m \cdot 2^{k}}$. Let $R \leq O(M+K+\log (1 / \beta))$ and $h:\{0,1\}^{k N} \times\{0,1\}^{s} \times\{0,1\}^{R} \rightarrow\{0,1\}$ be the respective quantities from Fact 4.19. Let $r:=R$. 
Let $T_{1}, \ldots, T_{\left(\begin{array}{c}N \\ m\end{array}\right)}$ be a list of all $m$-element subsets of $[N]$, in lexicographical order. Identifying $w \in\{0,1\}^{2^{s}}$ with $w \in\left[2^{2^{s}}\right]=\left\{1, \ldots, 2^{2^{s}}\right\}$, for $j \in\left[\left(\begin{array}{c}N \\ m\end{array}\right)\right]$, define

$$
W_{j}:=\left\{w \in\{0,1\}^{2^{s}} \mid 1+\left(w-1 \bmod \left(\begin{array}{l}
N \\
m
\end{array}\right)\right)=j\right\} .
$$

Observe that the sets $W_{j}$ partition $\{0,1\}^{2^{s}}$, and each has size $\left\lfloor 2^{2^{s}} /\left(\begin{array}{l}N \\ m\end{array}\right)\right\rfloor$ or $\left[2^{2^{s}} /\left(\begin{array}{c}N \\ m\end{array}\right)\right]$.

Given $\bar{y} \in\left(\{0,1\}^{N}\right)^{k}$ and random string $\rho \in\{0,1\}^{r}$, we define

$$
\psi_{k, N}(\bar{y}, \rho):=T_{j} \quad \text { where } \quad(h(\bar{y}, v, \rho))_{v \in\{0,1\}^{s}} \in W_{j} .
$$

We write $\psi$ for $\psi_{k, N}$. For $\rho \in\{0,1\}^{r}$, let $\psi^{\rho}:\left(\{0,1\}^{N}\right)^{k} \rightarrow\left(\begin{array}{c}{[N]} \\ m\end{array}\right)$ be the function obtained from $\psi$ by setting its random seed to $\rho$.

Since $h$ is polynomial time computable and $s=O\left(\log \left(6 \cdot \log 2^{N}\right)\right)=O(\log N)$, we see that $\psi$ is also polynomial time computable.

Furthermore, we claim that $\psi$ satisfies the property that we need: for $2^{k}$ distinct points $\bar{y} \in\left(\{0,1\}^{N}\right)^{k}$, the evaluations of $\psi$ over the choice of the random seed $\rho$ are close to uniformly distributed.

Lemma 4.21. For large enough $N$, the following holds. Let $\left(\bar{y}^{u}\right)_{u \in\{0,1\}^{k}}$ be a collection of $2^{k}$ distinct points in $\left(\{0,1\}^{N}\right)^{k}$. Then,

$$
\left|\psi\left(\left(\bar{y}^{u}\right)_{u \in\{0,1\}^{k}}, \mathcal{U}\left(\{0,1\}^{r}\right)\right)-\mathcal{U}\left(\left(\begin{array}{c}
{[N]} \\
m
\end{array}\right)^{2^{k}}\right)\right| \leq 2 \cdot \beta .
$$

Proof of Lemma 4.21. Consider an arbitrary event $E \subseteq\left(\begin{array}{c}{[N]} \\ m\end{array}\right)^{2^{k}}$. When picking a collection $\left(S_{u}\right)_{u \in\{0,1\}^{k}}$ uniformly at random from $\left(\begin{array}{c}{[N]} \\ m\end{array}\right)^{2^{k}}, \operatorname{Pr}\left[\left(S_{u}\right)_{u \in\{0,1\}^{k}} \in E\right]=|E| /\left(\begin{array}{c}N \\ m\end{array}\right)^{2^{k}}$. Therefore, we want to show that

$$
\left|\operatorname{Pr}_{\rho}\left[\left(\psi\left(\bar{y}^{u}, \rho\right)\right)_{u \in\{0,1\}^{k}} \in E\right]-\frac{|E|}{\left(\begin{array}{l}
N \\
m
\end{array}\right)^{2^{k}}}\right| \leq 2 \cdot \beta .
$$

Recall the sets $T_{j}$ and $W_{j}$ from Definition 4.20. Consider any point $\left(T_{j_{u}}\right)_{u \in\{0,1\}^{k}} \in E$. For every $u, \psi\left(\bar{y}^{u}, \rho\right)=T_{j_{u}}$ if and only if $\left(h\left(\bar{y}^{u}, v, \rho\right)\right)_{v \in\{0,1\}^{s}} \in W_{j_{u}}$. We write

$$
\prod_{u \in\{0,1\}^{k}} W_{j_{u}}:=\left\{w \in\left(\{0,1\}^{2^{s}}\right)^{2^{k}} \mid \forall u \in\{0,1\}^{k}, w_{u} \in W_{j_{u}}\right\} .
$$


Define the event

$$
E^{\prime}:=\bigcup_{\left(T_{j_{u}}\right)_{u \in\{0,1\}^{k}} \in E}\left(\prod_{u \in\{0,1\}^{k}} W_{j_{u}}\right) \subseteq\left(\{0,1\}^{2^{s}}\right)^{2^{k}} .
$$

Observe that with these definitions,

$$
\left(\psi\left(\bar{y}^{u}, \rho\right)\right)_{u \in\{0,1\}^{k}} \in E \quad \Longleftrightarrow \quad\left(\left(h\left(\bar{y}^{u}, v, \rho\right)\right)_{v \in\{0,1\}^{s}}\right)_{u \in\{0,1\}^{k}} \in E^{\prime} .
$$

We write

$$
P:=\operatorname{Pr}_{\rho}\left[\left(\psi\left(\bar{y}^{u}, \rho\right)\right)_{u \in\{0,1\}^{k}} \in E\right]=\operatorname{Pr}_{\rho}\left[\left(\left(h\left(\bar{y}^{u}, v, \rho\right)\right)_{v \in\{0,1\}^{s}}\right)_{u \in\{0,1\}^{k}} \in E^{\prime}\right]
$$

When picking a string $w \in\left(\{0,1\}^{2^{s}}\right)^{2^{k}}$ uniformly at random, $\operatorname{Pr}\left[w \in E^{\prime}\right]=$ $\left|E^{\prime}\right| / 2^{2^{k} 2^{s}}$. Therefore, by Fact 4.19 ,

$$
\left|P-\frac{\left|E^{\prime}\right|}{2^{2^{k} 2^{s}}}\right| \leq \beta
$$

Furthermore, since for every $\left.j, \mid 2^{2^{s}} /\left(\begin{array}{c}N \\ m\end{array}\right)\right\rfloor \leq\left|W_{j}\right| \leq\left\lceil 2^{2^{s}} /\left(\begin{array}{c}N \\ m\end{array}\right)\right]$, we get

$$
|E| \cdot\left(\frac{2^{2^{s}}}{\left(\begin{array}{c}
N \\
m
\end{array}\right)}-1\right)^{2^{k}} \leq\left|E^{\prime}\right| \leq|E| \cdot\left(\frac{2^{2^{s}}}{\left(\begin{array}{c}
N \\
m
\end{array}\right)}+1\right)^{2^{k}} .
$$

Combining these, we get

$$
|E| \cdot\left(\frac{1}{\left(\begin{array}{c}
N \\
m
\end{array}\right)}-\frac{1}{2^{2^{s}}}\right)^{2^{k}}-\beta \leq P \leq|E| \cdot\left(\frac{1}{\left(\begin{array}{c}
N \\
m
\end{array}\right)}+\frac{1}{2^{2^{s}}}\right)^{2^{k}}+\beta
$$

Using $(a+b)^{c} \leq a^{c}+2^{c} \cdot b$ and $(a-b)^{c} \geq a^{c}-2^{c} \cdot b$ for $0<a, b<1$, and $|E| \leq\left(\begin{array}{c}N \\ m\end{array}\right)^{2^{k}}$, we obtain

$$
\frac{|E|}{\left(\begin{array}{l}
N \\
m
\end{array}\right)^{2^{k}}}-\left(\frac{2 \cdot\left(\begin{array}{l}
N \\
m
\end{array}\right)}{2^{2^{s}}}\right)^{2^{k}}-\beta \leq P \leq \frac{|E|}{\left(\begin{array}{l}
N \\
m
\end{array}\right)^{2^{k}}}+\left(\frac{2 \cdot\left(\begin{array}{c}
N \\
m
\end{array}\right)}{2^{2^{s}}}\right)^{2^{k}}+\beta .
$$

Since $s=\left\lceil\log \log \left(\left(\begin{array}{c}N \\ m\end{array}\right)^{6}\right)\right], 2 \cdot\left(\begin{array}{c}N \\ m\end{array}\right) / 2^{2^{s}} \leq 1 /\left(\begin{array}{c}N \\ m\end{array}\right)^{4} \leq\left(m^{4} / N^{2}\right)^{m} \cdot 1 / N^{2 m}=N^{2(e-1) m}$. $1 / N^{2 m} \leq 1 / N^{2 m}$ for large enough $N$. Therefore,

$$
\left|P-\frac{|E|}{\left(\begin{array}{l}
N \\
m
\end{array}\right)^{2^{k}}}\right| \leq\left(\frac{1}{N^{2 m}}\right)^{2^{k}}+\beta=2 \cdot \beta
$$


We are now in a position to prove an alternative to Lemma 4.17 for the new selection function $\psi$.

Lemma 4.22. For large enough $N$, the following holds. For every $q>0$, uniformly chosen $\bar{y}^{0}, \bar{y}^{1} \in\left(\{0,1\}^{N}\right)^{k}$ and random $\rho \in\{0,1\}^{r}$,

$$
\operatorname{Pr}_{\bar{y}^{0}, \bar{y}^{1}, \rho}[q(\mathcal{S})=q] \leq\left(\frac{m^{3} \cdot 2^{2^{k}}}{q \cdot N}\right)^{q},
$$

where $\mathcal{S}:=\left(S_{u}\right)_{u \in\{0,1\}^{k}}, S_{u}:=\psi\left(\bar{y}^{u}, \rho\right) \in\left(\begin{array}{c}{[N]} \\ m\end{array}\right)$, and $\bar{y}^{u}:=\left(y_{1}^{u_{1}}, \ldots, y_{k}^{u_{k}}\right)$.

Proof of Lemma 4.22. As in the proof of Lemma 4.17, the probability that there exists an $i \in[k]$ such that $y_{i}^{0}=y_{i}^{1}$ is at most $k / 2^{N}$. Conditioned on this not occurring, the $2^{k}$ points $\bar{y}^{u}$ are distinct. If the collection $\mathcal{S}$ is picked uniformly at random, Lemma 4.18 gives a bound for the probability of the event that $q(\mathcal{S})=q$. By Lemma 4.21, when the collection $\mathcal{S}$ is picked using the selection function $\psi$ and a random $\rho$, the distribution is $(2 \cdot \beta)$-close to the uniform distribution, hence

$$
\operatorname{Pr}_{\bar{y}^{0}, \bar{y}^{1}, \rho}\left[q(\mathcal{S})=q \mid \forall i, y_{i}^{0} \neq y_{i}^{1}\right] \leq\left(\frac{e \cdot m^{2} \cdot 2^{2 k}}{q \cdot N}\right)^{q}+2 \cdot \beta .
$$

Therefore,

$$
\begin{aligned}
\operatorname{Pr}_{\bar{y}^{0}, \bar{y}^{1}, \rho}[q(\mathcal{S})=q] & \leq \operatorname{Pr}_{\bar{y}^{0}, \bar{y}^{1}}\left[\exists i \in[k], y_{i}^{0}=y_{i}^{1}\right]+\operatorname{Pr}_{\bar{y}^{0}, \bar{y}^{1}, \rho}\left[q(\mathcal{S})=q \mid \forall i, y_{i}^{0} \neq y_{i}^{1}\right] \\
& \frac{k}{2^{N}}+\left(\frac{e \cdot m^{2} \cdot 2^{2 k}}{q \cdot N}\right)^{q}+2 \cdot\left(\frac{1}{N}\right)^{2 m 2^{k}} \\
& \leq\left(\frac{m^{3} \cdot 2^{2^{k}}}{q \cdot N}\right)^{q} .
\end{aligned}
$$

For the last inequality above, we first use $k / 2^{N} \leq\left(e \cdot m^{2} \cdot 2^{2 k} / q \cdot N\right)^{q}$ as in the proof of Lemma 4.17). Then, $1 / N^{2 m \cdot 2^{k}}=\left(1 / N^{2}\right)^{m \cdot 2^{k}} \leq\left(e \cdot m^{2} \cdot 2^{2 k} / q \cdot N\right)^{q}$ since $q \leq m \cdot 2^{k} \leq N$. Finally, $4 \cdot\left(e \cdot m^{2} \cdot 2^{2 k} / q \cdot N\right)^{q} \leq\left(4 \cdot e \cdot m^{2} \cdot 2^{2 k} / q \cdot N\right)^{q} \leq\left(m^{3} \cdot 2^{2 k} / q \cdot N\right)^{q}$ since $q \geq 1$.

Since the bound in Lemma 4.22 is exactly the same as the one in Lemma 4.17, by the exact same derivation as in the proof of Theorem 4.10, we obtain the following derandomized equivalent. 
Theorem 4.23. For every $\delta<1$ and for every function $k^{\prime}=k^{\prime}(N)$ satisfying $k^{\prime} \leq \delta \cdot \log N$ for all sufficiently large $N$, there are constants $\epsilon, \alpha>0$ such that, for $m:=N^{\epsilon}$ and for all sufficiently large $N$, for $r$ and $\psi_{k, N}^{\rho}$ as in Definition 4.20, the following holds. There is a distribution $\lambda$ on $\left(\{0,1\}^{N}\right)^{k+1}$ such that

$$
\underset{\rho \sim \mathcal{U}\left(\{0,1\}^{r}\right)}{\mathrm{E}}\left[\operatorname{corr}_{\lambda}\left(\operatorname{Lift}\left(\mathrm{OR}_{m}, \psi_{k, N}^{\rho}\right), \Pi_{k+1, N}^{N^{\alpha}}\right)\right]<1 / 3 .
$$

As the final piece towards the proof of the explicit separation, we argue that even if the random seed $\rho$ is included as part of the input, the function is still hard for NOF randomized protocols.

Proof of Theorem 4.1. We use $N^{*}$ for the input size to the explicit function we construct, so $k+1 \leq \delta \cdot \log N^{*}$ for $\delta<1$.

Consider the function $F_{k+1, N^{*}}^{*}:\left(\{0,1\}^{N^{*}}\right)^{k+1} \rightarrow\{-1,+1\}$ defined as follows. Let $N:=\sqrt{N^{*}} / k$. Let $r=r(N)$ be defined as in Theorem 4.23 and Definition 4.20, On inputs $z_{0}, z_{1}, \ldots, z_{k} \in\{0,1\}^{N^{*}}, F_{k+1, N^{*}}^{*}\left(z_{0}, z_{1}, \ldots, z_{k}\right):=1$ unless:

(i) $z_{0}=x \circ 0^{N^{*}-N}$;

(ii) $z_{1}=y_{1} \circ \rho \circ 0^{N^{*}-N-r}$;

(iii) $z_{i}=y_{i} \circ 0^{N^{*}-N}$ for $2 \leq i \leq k$; and

(iv) $\operatorname{Lift}\left(\mathrm{OR}_{m}, \psi_{k, N}^{\rho}\right)\left(x, y_{1}, \ldots, y_{k}\right)=-1$.

We need to check that we can indeed embed the random seed as part of the input to player 1. By Definition 4.20, $r=O\left(k \cdot N+2^{k} \cdot 2^{s}+2 \cdot m \cdot 2^{k} \cdot \log N\right)$. First, $k \cdot N \leq \sqrt{N^{*}}$. Second, $2^{k} \cdot 2^{s} \leq\left(N^{*}\right)^{\delta} \cdot 2 \cdot 6 \cdot \log \left(\begin{array}{l}N \\ m\end{array}\right) \leq O\left(\left(N^{*}\right)^{\delta} \cdot m \cdot \log N\right)=O\left(\left(N^{*}\right)^{\delta} \cdot N^{\epsilon} \cdot \log N\right) \leq$ $O\left(\left(N^{*}\right)^{\delta+\epsilon} \log N^{*}\right)$. Third, $m \cdot 2^{k} \cdot \log N \leq O\left(\left(N^{*}\right)^{\delta+\epsilon} \log N^{*}\right)$. Putting these together, $r=O\left(\left(N^{*}\right)^{\max \{1 / 2, \delta+2 \epsilon\}}\right)=o\left(N^{*}\right)$ when $\epsilon=(1-\delta) / 5$. Therefore, when $N^{*}$ is large enough, $r \leq N^{*} / 2$. Since $N \leq \sqrt{N^{*}} \leq N^{*} / 2$, we get $r+N \leq N^{*}$, so the input of player 1 fits on $N^{*}$ bits.

The assertion that $F^{*}$ is polynomial time computable follows from the fact that $\psi$ is polynomial time computable. 
Furthermore, $F_{k+1, N^{*}}^{*}$ still has an efficient nondeterministic protocol: player 0 sees both the random seed $\rho$, and all of $y_{1}, \ldots, y_{k}$, so it can compute $\psi_{k, N}^{\rho}\left(y_{1}, \ldots, y_{k}\right) \in\left(\begin{array}{c}{[N]} \\ m\end{array}\right)$; player 0 guesses an index $i \in \psi_{k, N}^{\rho}\left(y_{1}, \ldots, y_{k}\right)$ and communicates it on $O(\log N)=$ $O\left(\log N^{*}\right)$ bits; player 1 checks that $x_{i}=1$. Thus, $N_{k+1}\left(F_{k+1, N^{*}}^{*}\right) \leq O\left(\log N^{*}\right)$.

Lastly, let $\lambda^{\prime}$ be the distribution on $\left(\{0,1\}^{N^{*}}\right)^{k+1}$ where $\left(x, y_{1}, \ldots, y_{k}\right)$ are distributed as in $\lambda$ (from Theorem 4.23) and, independently, $\rho$ is distributed uniformly. Using the inequality $\max _{a} \mathrm{E}_{b}[f(a, b)] \leq \mathrm{E}_{b}\left[\max _{a} f(a, b)\right]$, we get

$$
\begin{aligned}
\operatorname{corr}_{\lambda^{\prime}}\left(F_{k+1, N^{*}}^{*}, \Pi_{k+1, N^{*}}^{N^{\alpha}}\right) & =\max _{\pi \in \Pi_{k+1, N^{*}}^{N^{\alpha}}} \operatorname{corr}_{\lambda^{\prime}}\left(F_{k+1, N^{*}}^{*}, \pi\right) \\
& =\max _{\pi \in \Pi_{k+1, N^{*}}^{N^{\alpha}}} \underset{\sim \mathcal{U}\left(\{0,1\}^{r}\right)}{\mathrm{E}} \underset{(x, \bar{y}) \sim \lambda}{\mathrm{E}}\left[\left(\operatorname{Lift}\left(\mathrm{OR}_{m}, \psi_{k, N}^{\rho}\right) \cdot \pi\right)(x, \bar{y})\right] \\
& \leq \underset{\rho \sim \mathcal{U}\left(\{0,1\}^{r}\right)}{\mathrm{E}}\left[\max _{\pi \in \Pi_{k+1, N^{*}}^{N^{\alpha}}} \underset{(x, \bar{y}) \sim \lambda}{\mathrm{E}}\left[\left(\operatorname{Lift}\left(\mathrm{OR}_{m}, \psi_{k, N}^{\rho}\right) \cdot \pi\right)(x, \bar{y})\right]\right] \\
& =\underset{\rho \sim \mathcal{U}\left(\{0,1\}^{r}\right)}{\mathrm{E}}\left[\operatorname{corr}_{\lambda}\left(\operatorname{Lift}\left(\mathrm{OR}_{m}, \psi_{k, N}^{\rho}\right), \Pi_{k+1, N^{*}}^{N^{\alpha}}\right)\right] \\
& <1 / 3 .
\end{aligned}
$$

By Fact 4.6, $R_{k+1}\left(F_{k+1, N^{*}}^{*}\right)>N^{\alpha}$. But $N=\sqrt{N^{*}} / k \geq \sqrt{N^{*}} / \log N^{*} \geq\left(N^{*}\right)^{1 / 3}$ since $k \leq \log N^{*}$. Hence, $R_{k+1}\left(F_{k+1, N^{*}}^{*}\right)>\left(N^{*}\right)^{\alpha / 3}$.

\subsection{Discussion}

In this chapter, we extended the exponential separation between randomized and nondeterministic $k$-player NOF protocols from $k<\log \log n$ [LS08, CA08] to $k<\delta \cdot \log n$ for every $\delta<1$. To achieve this, we built on the breakthrough work of [She09, She08b], which connects communication complexity with the representation of boolean functions by real polynomials.

We started with a $((k+1) \cdot N)$-bit lifted function Lift $(\mathrm{OR}, \phi)$ that consists of the base function $\mathrm{OR}=\mathrm{OR}_{m}$ and a random selection function $\phi=\phi_{k, N}$. Using the 
approximation-orthogonality principle of [She08b], we obtained a function $g$ highly correlated to OR, and perpendicular to all Fourier characters of small sets. Applying the generalized discrepancy method [Kla07, Raz03], we reduced the problem of proving lower bounds for randomized protocols for $\operatorname{Lift}(\mathrm{OR}, \phi)$ to that of proving upper bounds for the discrepancy of $\operatorname{Lift}(g, \phi)$. Using [BNS92], we analyzed the latter in terms of the average product of $2^{k}$ evaluations of $g$ on a family $\mathcal{S}$ of $m$-element subsets of $[n]$. The heart of the argument was that the random choice of $\phi$ translates into the sets in $\mathcal{S}$ being "spread out", enough so that the fact $g$ is perpendicular to small Fourier characters implies good discrepancy upper bounds for $\operatorname{Lift}(g, \phi)$.

The dependency on the number of players in our lower bound is optimal, because of our reliance on the discrepancy method of [BNS92], which stops working as soon as $k=\log n$. It remains a major open problem in communication complexity to prove any lower bound for $k$-player NOF randomized protocols, even for $k=\log n$. As mentioned in Section 3.6, it would be interesting to study whether better (in terms of the dependency on $k$ ) separations between randomized and nondeterministic protocols could be achieved by counting arguments.

In this work, we showed that the separation between randomized and nondeterministic protocols can be achieved by a polynomial time computable function. This result has been subsequently improved by [BHN09], who showed that the separation can be achieved by a function computed by small depth $\left(\mathrm{AC}^{0}\right)$ circuits. They also improved the lower bounds for the Set Intersection function from $k<\log \log n$ to $k<$ $\Theta\left((\log n)^{1 / 3}\right)$. It would be interesting to extend this lower bound to $k<\Theta(\log n)$.

Randomized lower bounds for functions with cheap nondeterministic protocols are relevant to proof complexity. [BPS07, BPH09] showed how to use reductions from NOF communication complexity to obtain lower bounds for proof systems in which the lines are degree- $r$ inequalities, for $r<\log \log n$. Intuitively, the degree $r$ corresponds to the number of players $k$ in the protocol that is simulating an efficient proof. 
For that reason, it seems it could be possible to prove lower bounds for $r<(\log n)^{\Theta(1)}$ or even $r<\Theta(\log n)$. 


\section{Chapter 5}

\section{Trade-off Lower Bounds for Stack}

\section{Machines}

In this section, we present the third major contribution of this work, which is a new reduction that allows us to use communication complexity lower bounds to derive certain trade-off lower bounds for the computational power of a Turing Machine equipped with a stack.

Henceforth, a Stack Machine (SM) is a Turing Machine with a single "external" or "input" read-only tape containing the input, a fixed number of "internal" or "work" read-write tapes, and a stack. The stack is itself realized as another one-way infinite tape that follows the access semantics of a stack [Coo71]. When dealing with SMs, a space bound refers exclusively to the size of the work tapes, and a pass bound refers exclusively to the number of passes allowed on the input tape. Thus, neither a space bound nor a pass bound places any restriction on the way a SM accesses its stack. Furthermore, we assume that a SM can keep track of the position of its head on the input tape, and that in every pass over the input tape, the head travels from one end of the tape to the

other. 1 For reasons laid out in the Remark below, we concentrate on the setting when

\footnotetext{
${ }^{1}$ When a TM is allowed two-way access to a tape, it is also standard to measure the number of times
} 
SMs are allowed two-way access to the input tape.

Remark 5.1 (One-way vs. Two-way Access). Clearly, two-way access is more general than one-way access. Furthermore, note that the two regimes are polynomially (yet, super-linearly) equivalent. A TM $M$ (with or without a stack) that makes $r(N)$ two-way passes over an input of size $N$ can be simulated by a similar TM $M^{\prime}$ that makes $r(N) \cdot N$ one-way passes: every time $M$ has to move the head left, $M^{\prime}$ pauses the simulation of $M$, starts a new one-way pass, and scans to the appropriate tape location. This is possible because, by assumption, $M^{\prime}$ has enough space to store in its work tape the location of its head on the input tape. Thus, every one of the $r(N)$ passes of $M$ is replaced by at most $N$ one-way passes of $M^{\prime}$.

However, the method presented in this work for proving trade-off lower bounds bottoms up at $\Omega(N)$ because the communication complexity of every function is $O(N)$. In this setting, the best lower bounds we can hope for, for either one-way or two-way access, are of the form $r(N) \geq \Omega\left(N^{\alpha}\right)$ for some $0<\alpha \leq 1$. Therefore, we cannot use our current methods to prove lower bounds for one-way access and then transfer them to two-way access using the simple argument above. Because of this reason, we concentrate directly on the setting when SMs are allowed two-way access.

The main technical result in this chapter is algorithmic in nature, specifically, we show that a $k$-player NIH communication protocol for a base function $f=f_{k, n}$ : $\left(\{0,1\}^{n}\right)^{k} \rightarrow\{0,1\}$ can efficiently simulate a SM for a related function $F=F_{k, m \cdot n}$ : $\left(\left(\{0,1\}^{n}\right)^{m}\right)^{k} \rightarrow\{0,1\}$, which consists, informally, of $m$ instances of $f$, "glued together" by a combining function $h:\{0,1\}^{m} \rightarrow\{0,1\}$ that is symmetrical and has a neutral element (e.g., this is the case for OR and XOR, with neutral element 0 ). We informally state the result here, and we defer the formal definition of $F$ to Section 5.2 .

it reverses the direction of movement of its head on that tape. The connection between this measure and the one we use in this work is: the number of passes equals one plus the number of tape head reversals. 
Theorem 5.2 (Informal statement). Let $k=k(n) \geq 2$ be a non-decreasing function and let $m=m(n) \geq 1$ be an increasing function such that $k \leq m^{O(1)}$. Let $N=N(n):=k \cdot m \cdot n$. Let $s=s(N)$ and $r=r(N)$ be increasing functions and let $\delta<1 / 2$ be a constant. Let $d:=k \cdot r \cdot \log r / \sqrt{m}$.

Let $f=f_{k, n}$ be a boolean base function, and let $F=F_{N}$ be a function related to $f$ in the sense informally described above, and made precise in Section [5.2.

Assume there exists a randomized SM for F with space bound s, pass bound $r$ and error $\delta$. Then, there exists a randomized protocol for $f$, with $\operatorname{cost} O(k \cdot r \cdot \log (k \cdot r) \cdot s)$ and error at most $\delta+O(d)$.

The proof of Theorem 5.2 follows the general line of argument used in [BHN08] to obtain a similar result for the related, but incomparable, setting when, instead of an unbounded stack, the Turing Machine has access to a constant number of external read-write tapes, and the number of passes is counted on all of these tapes.

As a consequence of Theorem 5.2 and of the known communication complexity lower bound for the Inner Product function, we obtain the following trade-off lower bound.

Corollary 5.3 (Informal statement). Let $\epsilon>0$ and $\delta<1 / 2$ be constants. There exists an "inner product-like" function $F=F_{N}$ such that any $S M$ computing $F$ with error $\delta$ requires space $s=\omega\left(N^{1 / 4-\epsilon}\right)$ or passes $r=\omega\left(N^{1 / 4-\epsilon}\right)$.

In particular, a log-space SM needs $\omega\left(N^{1 / 4-\epsilon}\right)$ passes to compute $F$.

The $\ell$-th frequency moment of a sequence $\bar{a}=\left(a_{1}, \ldots, a_{t}\right)$, where $a_{i} \in[R]$, is

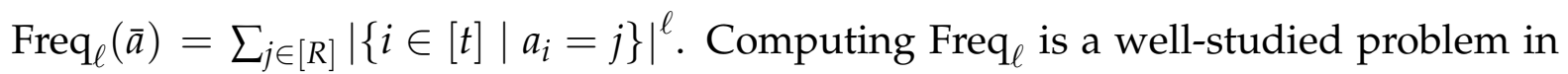
the streaming literature [AMS99]. As another consequence of Theorem 5.2, we also obtain the following result, which can be interpreted as saying that a stack does not help in computing frequency moments. 
Corollary 5.4. Let $\ell>4, \epsilon \geq 0$ and $\delta<1 / 2$ be constants. There exists a constant $0<\beta<1$ such that any randomized SM computing a $(1+\epsilon)$ multiplicative approximation of $\mathrm{Freq}_{\ell}$ with error $\delta$ requires space $s=\omega\left(N^{\prime} \beta\right)$ or passes $r=\omega\left(N^{\prime \beta}\right)$, where $N^{\prime}$ denotes the input size.

In particular, a log-space $S M$ needs $\omega\left(N^{\prime} \beta\right)$ passes to approximate Freq $_{\ell}$.

For this result, we use a NIH communication complexity lower bound for the promise Set Intersection function [Gro09], along with a streaming reduction between the problems of computing the promise Set Intersection function and the frequency moments of a data stream, originating from [AMS99].

We begin in Section 5.1 with an informal argument outlining the proof of Theorem 5.2 in a simplified setting that still involves most of the difficulties. We formally define the notion of "permuted functions" in Section 5.2. In Section 5.3, we give the proof of Theorem 5.2 based on two key Lemmas. We defer their proofs to Sections 5.4 and 5.5. Finally, in Section 5.6 we prove Corollaries 5.3 and 5.4 .

Throughout this section, we only consider NIH communication protocols. The work in this Section appears in [DPS09].

\subsection{Outline of the Argument}

Consider the regular Inner Product function family $\mathrm{IP}=\operatorname{IP}_{2, n}:\left(\{0,1\}^{n}\right)^{2} \rightarrow\{0,1\}$ (see Section 2.3). Let $\left(x_{1}, x_{2}\right)$ be an input, with $x_{p}=\left(x_{p, 1}, \ldots, x_{p, n}\right)$, where $x_{p, j} \in\{0,1\}$ for $p \in[2]$ and $j \in[n]$. In the communication complexity world, we are interested in computing this function with 2 player protocols, in which player $p$ gets $x_{p}$, for $p \in[2]$. We know that $R_{2}\left(\mathrm{IP}_{2, n}\right) \geq \Omega(n)$ [CG88]. In the TM world, we are interested in computing this function when its input is given on a tape in the natural way: first the $n$ bits of $x_{1}$, then the $N$ bits of $x_{2}$. The input size for the TM is $N=2 \cdot n$. In this simplified outline, we study the trade-off between the space $s(N)$ and the number of one-way passes $r(N)$ that a deterministic TM needs. 
In the absence of a stack. To begin with, suppose we are dealing with a regular TM (with no stack). In this case, there is a simple, well-known, simulation of a space and pass bounded TM by a 2 player communication protocol. We include it here for reference.

Player 1, knowing $x_{1}$, simulates the TM until the head first crosses into $x_{2}$, which is the input seen by player 2; at this point player 1 communicates the space of the TM; player 2 continues the simulation until the head reaches the end of the input tape; at this point player 2 communicates the space of the TM; player 1 now continues the simulation of the next pass; at the end of the last pass, player 2 announces the output.

In total, the communication in this protocol is $(2 \cdot s(N)) \cdot(r(N)-1)+s(N)+1$. By the communication complexity lower bound, we get $s(N) \cdot r(N) \geq \Omega(n)=\Omega(N)$.

It is easy to see that the simulation above breaks down in the case of a SM because there might be transfer of information via the stack between the parts of the computation when the input head is scanning $x_{1}$ and $x_{2}$. Thus, to perform the simulation, the players might have to communicate the entire content of the stack, which is unbounded a priori. In order to be able to use communication complexity lower bounds for SMs, we need to show that a SM still solves efficiently some version of the hard communication problem.

Inner Product should still be hard for SMs with one-way access. Before we go on, we give some intuition suggesting that Inner Product is indeed hard for SMs with one-way access. First, consider the case of SMs with more powerful two-way access to the input. It is easy to see that such a SM can compute Inner Product with only 2 passes:

Push $x_{1}$ on the stack; go to the end of the tape; now, moving backwards on the input tape, read the symbols in $x_{2}$ while popping the symbols in 
$x_{1}$ from the stack. The ordering of those is now the same, so the SM can compute $x_{1, j} \wedge x_{2, j}$ for $j$ going from $N$ down to 1 , and keep their partial $\bmod 2$ sum in its state.

However, this scheme entirely breaks down when the SM is only allowed one-way access. Assume such a SM pushes all of $x_{1}$ on the stack during the first pass. Now, the moment when the input head is scanning $x_{2,1}$ (corresponding to the moment when player 2 first takes over the simulation), the value $x_{1,1}$ is at the bottom of the stack. Thus, if the SM attempted to retrieve it in order to compute $x_{1,1} \wedge x_{2,1}$ (which is one of the $n$ terms in the modulo 2 sum defining Inner Product), the entire stack contents on top of $x_{1,1}$ would be either lost or transferred to the (space bounded) work tapes. Then, by the time the SM got to, say, $x_{2, N / 2}$, the corresponding value $x_{1, N / 2}$ might not be available any more, so the machine would need another pass to retrieve it. While imprecise, this argument suggests that the ordering of the symbols on the stack plays an important role in the usefulness of the stack during the computation of a SM, to the extent that, even though a SM can push all of $x_{1}$ on the stack, it is still unclear how that could be used in order to compute Inner Product with small space and few one-way passes.

A framework for dealing with a stack. In order to deal with the presence of a stack, we adapt a framework that was originally introduced by [BHN08] in the context of TMs with several external tapes.

Consider a function $f$ which we know is hard in the communication model. We assume that an efficient SM $M$ exists for a related function $F$. Using this assumption, we build an efficient communication protocol $P$ for $f$. Let $x$ be an input to $f$ in $P$. The players in $P$ first construct an input $v$ to $F$ (which contains $x$ and some public "padding"), they simulate $M$ on $v$, computing $F(v)$, and finally they derive $f(x)$ from $F(v)$. The simulation of $M$ on $v$ must be efficient so that communication lower bounds 
apply.

Let $\Gamma$ be the sequence of configurations of $M$ on $v$. In $P$, the players simulate $\Gamma$ using a series of publicly and privately simulated sections. The public simulation is "cheap" in the sense that it does not cost any communication. The private simulation is "expensive" because, at the end of each private section, the player simulating it must communicate something in order for the other player(s) to "know where they

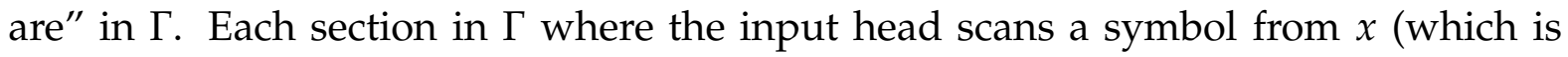
the input to $P$ ) is to be simulated privately by the player who knows that symbol. Moreover, the basic idea is that, since we do not have any bounds on how a SM can use its stack, we want to avoid communicating stack content in $P$. Thus, we do not mind if either:

- a symbol is pushed on the stack during public simulation and popped during either public or private simulation; or

- a symbol is pushed on the stack in a privately simulated section by player $p$ and later popped in a (possibly different) privately simulated section the same player $p$.

What we want to avoid is the remaining scenario:

- a symbol is pushed on the stack in a privately simulated section by player $p_{1}$ and later popped in a privately simulated section by player $p_{2} \neq p_{1}$.

Informally, the way we achieve this "protection" against $M$ using its stack on $x$ is by "hiding" $x$ into the larger input $v$, so that, with high probability, $M$ only uses its stack for "meaningless" computation.

More concretely, let $m=n=\sqrt{N / 2}$. Suppose that in a communication protocol 2 players want to compute $\mathrm{IP}_{2, n}$, and they have access to an efficient (space $s(N)$, one-way passes $r(N)) \mathrm{SM}$ for $\mathrm{IP}_{2, N / 2}$. We think of $\mathrm{IP}_{2, N / 2}$ as $m$ instances of $\mathrm{IP}_{2, n}$ glued 
together by a top-level $\mathrm{XOR}_{m}$ gate. That is, for an input $v=\left(v_{1}, v_{2}\right) \in\left(\{0,1\}^{N / 2}\right)^{2}$, let $v_{p}=\left(v_{p, 1}, \ldots, v_{p, m}\right)$ where $v_{p, i} \in\{0,1\}^{n}$ for $p \in[2], i \in[m]$. Observe that $\operatorname{IP}\left(v_{1}, v_{2}\right)=$ $\mathrm{XOR}_{m}\left(\operatorname{IP}\left(v_{1, i}, v_{2, i}\right) \mid i \in[m]\right)$.

In the communication protocol $P$, the players get inputs $x_{1}, x_{2} \in\{0,1\}^{n}$, respectively. They begin by choosing a random $i^{*} \in[m]$ and random $y_{1, i}, y_{2, i} \in\{0,1\}^{n}$ for $i \in[m] \backslash\left\{i^{*}\right\}$, all using public coins. Let $v=\left(v_{1}, v_{2}\right)$ be the input to $M$ defined by, $v_{p, i^{*}}:=x_{p}$ and $v_{p, i}:=y_{p, i}$, for $i \neq i^{*}$ and $p \in[2]$. Henceforth, the goal of $P$ is to simulate $M$ on $v$.

Thus, both players know most of the input to the SM, except for two pieces of $n$ bits each, where their respective inputs from the communication protocol are embedded in the $N$ bit input $v$ to $M$. Furthermore, observe that if they were indeed able to simulate the SM, they could compute the output for the protocol as

$$
\begin{aligned}
\operatorname{IP}\left(x_{1}, x_{2}\right) & =\operatorname{IP}\left(v_{1, i^{*},}, v_{2, i^{*}}\right) \\
& =\operatorname{XOR}_{2}\left(\operatorname{IP}\left(v_{1}, v_{2}\right), \operatorname{XOR}_{m-1}\left(\operatorname{IP}\left(v_{1, i}, v_{2, i}\right) \mid i \in[m] \backslash\left\{i^{*}\right\}\right)\right) .
\end{aligned}
$$

Unique stack symbols. For the purposes of the analysis, we assume that each symbol on the stack is given a unique tag. Thus, even though the same symbol might appear many times on the stack, we assume we can distinguish between any of those appearances. In particular, to say that "a symbol is placed on the stack in configuration $\gamma_{1}$ and popped in configuration $\gamma_{2}$ " formally means that:

- the stack level is the same in $\gamma_{1}$ and in the configuration immediately following $\gamma_{2} ;$ and

- the stack level is strictly higher in any intermediate configuration between those two.

Corrupted instances. A key concept in our argument is that of a "corrupted instance". For a SM M and an input $v$, we say that instance $i$ is corrupted in $v$ on $M$ 
if, during the run of $M$ on $v$, a symbol is placed on the stack while the input head is scanning $v_{p_{1}, i}$ and popped while it is scanning $v_{p_{2}, i}$, where $p_{1} \neq p_{2}$.

Three claims. Henceforth, our argument is built on the following three claims:

(i) The number of corrupted instances in any one input is small.

(ii) The choice of $i^{*}$ and of $v$ in the communication protocol are statistically independent of each other.

(iii) If the input $x$ to the communication protocol is not embedded in a corrupted instance in the input $v$ to the $\mathrm{SM}$, then the protocol can efficiently simulate the SM.

The intuition for claim (i). Fix a SM M and an input $v$. We want to give a bound for the number of corrupted instances in $v$ on $M$. We associate each corrupted instance $i \in$ $[m]$ with a unique 4 -tuple $\left(l_{1} \leq l_{2}, p_{1} \neq p_{2}\right)$, such that a symbol is pushed on the stack in the pass $l_{1}$ over $v_{p_{1}, i}$ and popped in the pass $l_{2}$ over $v_{p_{2}, i}$. Note, for each corrupted instance there might be several such 4-tuples to choose from, here, we associate $i$ with only (any) one of them. We say that $i$ is corrupted by this 4-tuple.

Let $l_{1} \leq l_{2} \leq r$ and let $p_{1} \neq p_{2} \in[2]$. During the pass $l_{1}$ over $v_{p_{1}}$, the input head scans the instances in $v$ going from left to right in the order: $1,2, \ldots, m$. The same is true for the pass $l_{2}$ over $v_{p_{2}}$. Assume instances $i_{1} \neq i_{2}$ are corrupted by this particular 4-tuple. Without losing generality, say $1 \leq i_{1}<i_{2} \leq m$. We see that: the stack symbol associated with $i_{1}$ is pushed first, then the stack symbol associated with $i_{2}$, then the stack symbol associated with $i_{1}$ is popped, and finally the stack symbol associated with $i_{2}$ is popped. This contradicts the First-In Last-Out access semantics of a stack. Hence, at most one instance is corrupted by any one 4-tuple.

Since the number of 4-tuples is at most $2 \cdot r^{2}$, we derive that at most $O\left(r^{2}\right)$ instances can be corrupted in $v$ on $M$. Thus, as long as $r^{2} / m=o(1)$, the fraction of corrupted 
instances in any one input is small. This argument is extended to work for two-way access, and slightly improved, in Section 5.4 .

The intuition for claim (ii). To see why we need (ii), observe that in the communication protocol $P, v$ is constructed based on $i^{*}$. For (i) to be useful, we would like the choice of $i^{*}$ to probabilistically "hide" the instance containing the input to $P$ from the set of corrupted instances in $v$ on $M$. It seems that we would need $i^{*}$ to be chosen after v.

To achieve (ii), we use an argument that goes through distributional communication complexity: for every distribution $D$ on the inputs to $\mathrm{IP}_{2, n}$ in the communication protocol, the players choose the $m-1$ "decoy" instances from that same distribution $D$, so that $v$ is distributed according to $D^{m}$ independently of $i^{*}$. In this case, we can permute the choices of $v$ and $i^{*}$, so that claim (i) gives a bound on the probability the communication protocol embeds the real input in a corrupted instance. We go back to randomized communication complexity (for which we have lower bounds) using the standard Yao's min-max principle [KN97, Theorem 3.20] connecting these two measures. This argument is made precise inside the proof of Theorem 5.2 in Section 5.3 .

The intuition for claim (iii). Assume that the instance $i^{*}$, where the real input $x=$ $\left(x_{1}, x_{2}\right)$ to the communication protocol $P$ is embedded in $v$, is not corrupted. We want to argue that $P$ can efficiently simulate the SM $M$.

Let $\Gamma$ be the sequence of configurations that $M$ goes through on input $v$. We say that a configuration is input-private to player $p$ if the input head is scanning a symbol from $v_{p, i^{*}}$ (which is where $x_{p}$ is embedded). Intuitively, we want player $p$ to simulate the transition out of a configuration that is input-private to player $p$, because only it knows the symbols in $v_{p, i^{*}}=x_{p}$. Moreover, symbols might be pushed on the stack in such a transition, we say that any such stack symbol is private to player $p$. We say that a configuration is stack-private to player $p$ if the transition out of this configuration pops 
a stack symbol that is private to player $p$. Intuitively, we want player $p$ to simulate such a transition in order to avoid communicating stack contents during the protocol. All other configurations are public. Since we assumed instance $i^{*}$ is not corrupted, we know there is no configuration which is input- and stack-private to different players.

The players simulate $\Gamma$ by alternating between public and private simulation. At the end of each privately simulated section, the player performing that simulation communicates: the state of the SM, the lowest stack level reached during the private simulation section, the current stack level, and the top stack symbol. By Fact 5.5, the height of the stack is at most $2^{O(s)}$, and hence the cost of the communication at the end of each privately simulated section is $O(s)$. In Section 5.5, we argue that the information communicated is sufficient for the players to obtain a "hollow view" of the stack: each player knows the public stack symbols, the stack symbols which are private to itself, and the locations of the stack symbols private to other players. Moreover, this hollow view is sufficient for the players to continue the simulation until the end of $\Gamma$.

Finally, let us give an informal bound on the amount of communication. Observe that input-private configurations form exactly $2 \cdot r$ contiguous subsequences in $\Gamma$. Let us denote these contiguous zones of input-private configurations in $\Gamma$ by $S_{a}$, for $1 \leq a \leq 2 \cdot r$. Let $\gamma_{a}$ and $\gamma_{a}^{\prime}$ be the configurations at the beginning and end of $S_{a}$, respectively. Observe that the stack at the end of $S_{a}$ (in $\gamma_{a}^{\prime}$ ) contains at most a contiguous zones of private stack symbols, at most one such zone corresponding to each previous input-private section $S_{a^{\prime}}$, with $a^{\prime} \leq a$. Some of these zones might be accessed before $S_{a+1}$. As explained in detail in Section 5.5, the players in the protocol can simulate the sequence of configurations between $\gamma_{a}^{\prime}$ and $\gamma_{a+1}$ using as many privately simulated sections as there are contiguous zones of private stack symbols in $\gamma_{a}^{\prime}$. Since $a \leq 2 \cdot r$, the number of privately simulated sections between $\gamma_{a}^{\prime}$ and $\gamma_{a+1}$ is $O(r)$. Summing over all $a$, and taking into account the privately simulated sections $S_{a}$, we get that, in total, the simulation of $\Gamma$ can be performed using at most $O\left(r^{2}\right)$ privately simulated sections. 
Hence, the communication bound for the entire protocol is $O\left(r^{2} \cdot s\right)$.

Putting (i), (ii) and (iii) together, assuming that we have a SM for $\mathrm{IP}_{2, N / 2}$, we obtain a randomized communication protocol for $\mathrm{IP}_{2, n}$ that has error $O\left(r^{2} / m\right)$ and cost $O\left(r^{2} \cdot s\right)$. As long as $r^{2} / m=o(1)$, the known randomized communication complexity lower bound $R_{2}\left(\mathrm{IP}_{2, n}\right) \geq \Omega(n)$ applies [CG88], and we obtain the trade-off lower bound $r(N)^{2} \cdot s(N) \geq \Omega(n)=\Omega\left(N^{1 / 2}\right)$.

What was left out of this outline. First, the number of players in this outline is set to $k=2$. While this is sufficient to derive SM trade-off lower bounds for Inner Productlike functions, we need to allow the number of players to be a function of the input size, that is, $k=k(n)$, in order to obtain inapproximability results for the frequency moments problem in Corollary 5.4 .

Second, the Inner Product function is of such a nature that no matter how the players in $P$ choose the decoy instances $y_{p, i} \in\{0,1\}^{n}$ for $p \in[2]$ and $i \neq i^{*}$, they can always retrieve $f(x)=\operatorname{IP}\left(x_{1}, x_{2}\right)$ from $F(v)=\operatorname{IP}\left(v_{1}, v_{2}\right)$ by computing the XOR of all decoy instances $\operatorname{IP}\left(y_{1, i}, y_{2, i}\right)$ together with $F(v)$. This is a property of the top-level gate in IP, which is XOR. In order to deal with the frequency moments problem, we use a reduction involving the promise Set Intersection problem, which has top-level gate OR. In this case, in order to be able to retrieve $f(x)$ from $F(v)$, the decoy instances must be 0 -instances, and only then do we have $f(x)=F(v)$. To accommodate for this requirement and still achieve claim (ii), we need to treat 0-inputs and 1-inputs differently inside the proof of Theorem 5.2 .

Third, we need to fill in the details of the simulation informally described as claim (iii).

Fourth, and most importantly, there is the issue of two-way versus one-way passes. As explained in Remark 5.1, to get trade-off lower bounds for SMs with two-way access, we need to have communication protocols directly simulate the latter. 
This outline only considers how to obtain trade-off lower bounds for SMs with one-way access to the input. As we have seen above, a SM with two-way access to the input can compute IP with only 2 passes. The point where this framework breaks down is claim (i), which is outright false. Consider the case when $p_{1}=1, p_{2}=2$, the pass $l_{1}=1$ is left-to-right, and the pass $l_{2}=2$ is right-to-left. We see that the instances are visited in the pass 1 in the order $1,2, \ldots, m-1, m$ and they are visited in the pass 2 in the order $m, m-1, \ldots, 2,1$. Then, the associated "corrupting" stack symbols can be pushed in the order $1, \ldots, m$ and popped in the order $m, \ldots, 1$, without violating the access semantics of a stack. Thus, potentially all instances can be corrupted by a single 4-tuple $(1,2,1,2)$, which is precisely what happens in the simple 2-pass protocol seen earlier.

In order to obtain trade-off lower bounds for SMs with two-way access, we need to fix claim (i). To that end, we "scramble" the order in which the $m$ instances are seen in pass $l_{1}$ over $v_{p_{1}}$ and in pass $l_{2}$ over $v_{p_{2}}$, no matter what $p_{1}$ and $p_{2}$ are, and no matter whether the passes $l_{1}$ and $l_{2}$ are left-to-right or right-to-left. Informally, we achieve this by reordering the $m$ instances corresponding to each player using a family of permutations $\Phi=\left(\varphi_{1}, \ldots, \varphi_{k}\right)$, with one permutation for each player, that has the following property.

For $p \in[k]$, let $\sigma_{p}:=\left(\varphi_{p}(1), \ldots, \varphi_{p}(m)\right)$ and $\sigma_{p}^{\text {rev }}:=\left(\varphi_{p}(m), \ldots, \varphi_{p}(1)\right)$. Then, for every $p_{1} \neq p_{2}, \sigma_{1}^{\prime} \in\left\{\sigma_{p_{1}}, \sigma_{p_{1}}^{\text {rev }}\right\}$, and $\sigma_{2}^{\prime} \in\left\{\sigma_{p_{2}}, \sigma_{p_{2}}^{\text {rev }}\right\}, \sigma_{1}^{\prime}$ and $\sigma_{2}^{\prime}$ have as small a common subsequence as possible.

It turns out that there exist families $\Phi$ where any such common subsequence has size $O(\sqrt{m})$ [BHN08]. 


\subsection{Preliminaries}

Notation. We use $n$ to denote the size of the input to a player in a communication protocol. The number of players is $k=k(n)$, which is a non-decreasing function of $n$. We use $f=f_{k, n}:\left(\{0,1\}^{n}\right)^{k} \rightarrow\{0,1\}$ to denote a generic boolean function which we are interesting in computing in the communication model.

We use $F$ to denote a generic function that we are interested in computing in the Stack Machine model. In general, $F$ consists of $m$ instances of a base function $f_{k, n}$ combined together by combining function $h_{m}:\{0,1\}^{m} \rightarrow\{0,1\}$. The number of instances, $m=m(n)$, is an increasing function of $n$. We denote by $N$ the size of the input to $F$, so $N=N(n)=k \cdot m \cdot n$. We use $s=s(N)$ and $r=r(N)$ to denote increasing functions bounding the space and the number of passes a SM is allowed (these are defined below).

Stack Machines. A deterministic (uniform) Stack Machine (SM) is a Turing Machine with a fixed number of internal tapes, a single read-only external tape of unbounded size containing the input, and a stack. The stack is implemented as an additional oneway infinite tape that follows the access semantics of a stack [Coo71]. A randomized SM is allowed to flip an unbiased coin before every single transition.

Let $s, r$ be increasing functions. A SM has a space bound $s$ if, for every $N$, the size of its work tapes is at most $s(N)$ on inputs of length $N$. A SM has a pass bound $r$ if, for every $N$, the number of passes it makes over the input tape is at most $r(N)$ on inputs of length $N$. Neither of these bounds refers in any way to the stack.

Without losing generality, we make the following assumptions about SMs:

(1) A SM has enough space on its work tapes to record the position of the head on the input tape. That is, $s(n) \geq \Omega(\log N)$.

(2) In every pass over the input tape, the input head travels from one end of the tape to the other. 
(3) The stack in a SM is empty both at the beginning and at the end of its computation.

(4) In every transition of a SM, exactly one of the following occurs:

- the input head moves on the input tape, referred to as a move transition; or

- a symbol is pushed on the stack, referred to as a push transition; or

- a symbol is popped from the stack, referred to as a pop transition.

It turns out that we need to consider non-uniform Stack Machines in one of our proofs. Naturally, the non-uniform model is more powerful than the uniform one, so lower bounds for the non-uniform model are stronger. Moreover, communication protocols are by definition a non-uniform model of computation, so when a communication protocol simulates a Stack Machine, we might as well assume the Stack Machine is also non-uniform.

For a fixed input length $N$, a stack automaton $A_{N}$ is an automaton with a read-only input tape of size $N$, a set of states whose size can now depend on $N$, and a stack. Assumptions (1) - (4) still apply. A stack automaton $A_{N}$ has a space bound $s(N)$ if $A_{N}$ has at most $2^{s(N)}$ states. $A_{N}$ has a pass bound $r(N)$ if $A_{N}$ makes at most $r(N)$ passes over its input.

A non-uniform Stack Machine is a collection $A=\left(A_{N}\right)_{N \in \mathbb{N}}$ of stack automata, one for each input length. For increasing functions $r, s$, a nonuniform SM $A=\left(A_{N}\right)$ has a space bound $s$ and a pass bound $r$ if, for every $N, A_{N}$ has a space bound $s(N)$ and a pass bound $r(N)$, respectively.

A (full) configuration of a SM contains:

- the state and the full contents of the work tapes in the uniform case, or simply the state in the non-uniform case;

- the location of the head on the input tape; and

- the entire contents of the stack. 
A surface configuration of a SM is the same as a full configuration, but instead of the entire stack contents, it only includes:

- the top stack symbol.

Fact 5.5. Let $A=\left(A_{N}\right)$ be a nonuniform SM that halts on every input and runs in space $s=s(N)$. Then, for large enough $N$ and for every input w of length $N$, the maximum stack height of $A$ during its run on $w$ is at most $2^{4 s(N)}$.

Proof of Fact 5.5. Assume the claim doesn't hold. Let $c$ denote the (constant) number of symbols in the stack alphabet of $A$. Let $N$ be large enough so that $s(N) \geq \log c$ (note that $s(N) \geq \log N$ by assumption (1)). Fix an input $w$ of size $N$ on which the stack reaches height more than $2^{4 s(N)}$. Let $\Gamma$ be the sequence of configurations of $M$ on $w$. Let $\gamma \in \Gamma$ be a configuration in which the stack contains strictly more than $2^{4 s(N)}$ symbols.

For every stack level $l$, with $1 \leq l \leq 2^{3 s(N)}+1<2^{4 s(N)}$, let $\gamma_{l}$ be the last configuration preceding $\gamma$ in which the symbol at level $l$ is at the top of the stack. Obviously, the transition out of $\gamma_{l}$ is a push transition. For every such $l$, let $\bar{\gamma}_{l}$ denote the surface configuration corresponding to $\gamma_{l}$.

Observe that a surface configuration can be represented on $\log N+s(N)+\log c \leq$ $3 \cdot s(N)$ bits. Since there are more than $2^{3 s(N)}$ symbols on the stack, a surface configuration must be repeated. Accordingly, let $1 \leq l_{1}<l_{2} \leq 2^{3 s(N)}+1$ be stack levels such that $\bar{\gamma}_{l_{1}}=\bar{\gamma}_{l_{2}}$

By definition, $\gamma_{l_{1}}$ is the last configuration when the stack level was $l_{1}$. Hence, the SM got from $\gamma_{l_{1}}$ to $\gamma_{l_{2}}$ without accessing a symbol at level $l_{1}$ or below. But then, since $\bar{\gamma}_{l_{2}}=\bar{\gamma}_{l_{1}}$, the SM is bound to repeat this part of its computation, eventually ending up in a configuration $\gamma_{l_{3}}$ with $l_{3}-l_{2}=l_{2}-l_{1}$ and $\bar{\gamma}_{l_{3}}=\bar{\gamma}_{l_{2}}$. This process is repeated indefinitely, so the SM does not halt on $w$, a contradiction. 
Permutations, Sequences, and Sortedness. Let $S_{m}$ denote the set of all permutations of $[m]$. Let id denote the identity permutation. For a permutation $\pi \in S_{m}$, let $\operatorname{seq}(\pi)$ denote the $m$-element sequence $(\pi(1), \pi(2), \ldots, \pi(m))$. For a sequence $\sigma$, let $\sigma^{\text {rev }}$ denote $\sigma$ reversed. E.g., seq $(\pi)^{\text {rev }}=(\pi(m), \ldots, \pi(1))$. For two sequences $\sigma_{1}, \sigma_{2}$, let $\operatorname{LCS}\left(\sigma_{1}, \sigma_{2}\right)$ denote their longest common (not necessarily contiguous) subsequence. For a sequence $\sigma$, let $|\sigma|$ denote its length.

Consider two permutations $\varphi_{1}, \varphi_{2} \in S_{m}$. We measure their relative sortedness according to the following experiment.

Intuitively, imagine we lay out seq $\left(\varphi_{1}\right)=\left(\varphi_{1}(1), \ldots, \varphi_{1}(m)\right)$ on one tape, and $\operatorname{seq}\left(\varphi_{2}\right)=\left(\varphi_{2}(1), \ldots, \varphi_{2}(m)\right)$ on another tape. We are allowed two passes, one over each tape, in parallel, with the heads moving independently of each other. We can choose to make the pass over each tape leftto-right or right-to-left. We "eliminate" an index $a \in[m]$ if, at some point during the two passes, $a$ is the value being scanned on both tapes. We define the relative sortedness of $\varphi_{1}$ and $\varphi_{2}$ to be the maximum number of indices we can eliminate in this way.

Formally,

$$
\text { relsort }\left(\varphi_{1}, \varphi_{2}\right):=\max \left\{\left|\operatorname{LCS}\left(\sigma_{1}, \sigma_{2}\right)\right| \mid \forall i \in[2], \sigma_{i} \in\left\{\operatorname{seq}\left(\varphi_{i}\right), \operatorname{seq}\left(\varphi_{i}\right)^{\mathrm{rev}}\right\}\right\}
$$

For example, for

$$
\varphi_{1}=\left(\begin{array}{llllll}
1 & 2 & 3 & 4 & 5 & 6 \\
\underline{2} & \underline{1} & \underline{4} & \underline{3} & 5 & 6
\end{array}\right) \text { and } \varphi_{2}=\left(\begin{array}{llllll}
1 & 2 & 3 & 4 & 5 & 6 \\
\underline{3} & 6 & \underline{4} & \underline{1} & 5 & \underline{2}
\end{array}\right),
$$

we have relsort $\left(\varphi_{1}, \varphi_{2}\right)=4$, because $(2,1,4,3)$ is a common subsequence of $\operatorname{seq}\left(\varphi_{1}\right)$ and $\operatorname{seq}\left(\varphi_{2}\right)^{\text {rev }}$.

For a set of permutations $\Phi=\left\{\varphi_{1}, \ldots, \varphi_{t}\right\}$, we define its relative sortedness to be the maximum relative sortedness of any two of its members. Formally,

$$
\operatorname{relsort}(\Phi):=\max \left\{\operatorname{relsort}\left(\varphi_{i}, \varphi_{j}\right) \mid i \neq j \in[t]\right\}
$$


We use the following Fact to obtain sets of permutations with small relative sortedness.

Fact 5.6. Let $\varphi_{1}, \varphi_{2} \in S_{m}$. If relsort $\left(\varphi_{1}, \varphi_{2}\right)=t$, then $\operatorname{seq}\left(\varphi_{2}^{-1} \circ \varphi_{1}\right)$ contains a monotone subsequence of length $t$.

Proof of Fact 5.6. First, assume $\left(i_{1}, i_{2}, \ldots, i_{t}\right)$ is a common subsequence of $\operatorname{seq}\left(\varphi_{1}\right)$ and $\operatorname{seq}\left(\varphi_{2}\right)$. In this case, the corresponding sequences of pre-images are both increasing, $\left(\varphi_{1}^{-1}\left(i_{1}\right)<\varphi_{1}^{-1}\left(i_{2}\right)<\ldots<\varphi_{1}^{-1}\left(i_{t}\right)\right)$ and $\left(\varphi_{2}^{-1}\left(i_{1}\right)<\varphi_{2}^{-1}\left(i_{2}\right)<\ldots<\varphi_{2}^{-1}\left(i_{t}\right)\right)$. Let $i_{j}^{\prime}:=\varphi_{1}^{-1}\left(i_{j}\right)$ for $j \in[t]$, and observe that $\varphi_{2}^{-1}\left(\varphi_{1}\left(i_{j}^{\prime}\right)\right)=\varphi_{2}^{-1}\left(i_{j}\right)$. Then, $\left(i_{1}^{\prime}, i_{2}^{\prime}, \ldots, i_{t}^{\prime}\right)$ is a subsequence of $(1,2, \ldots, m)$, so $\left(\varphi_{2}^{-1} \circ \varphi_{1}\left(i_{1}^{\prime}\right)<\varphi_{2}^{-1} \circ \varphi_{1}\left(i_{2}^{\prime}\right)<\ldots<\varphi_{2}^{-1} \circ \varphi_{1}\left(i_{t}^{\prime}\right)\right)$ is an increasing subsequence of $\left(\varphi_{2}^{-1} \circ \varphi_{1}(1), \varphi_{2}^{-1} \circ \varphi_{1}(2), \ldots, \varphi_{2}^{-1} \circ \varphi_{1}(m)\right)=\operatorname{seq}\left(\varphi_{2}^{-1} \circ\right.$ $\left.\varphi_{1}\right)$.

The cases when the subsequence $\left(i_{1}, i_{2}, \ldots, i_{t}\right)$ comes from seq $\left(\varphi_{1}\right)^{\text {rev }}$ instead of $\operatorname{seq}\left(\varphi_{1}\right)$, or from seq $\left(\varphi_{2}\right)^{\text {rev }}$ instead of $\operatorname{seq}\left(\varphi_{2}\right)$, or both, are treated similarly. The only difference is that one or both of the corresponding sequences of pre-images are now decreasing, which might change the monotonicity of the resulting subsequence of $\operatorname{seq}\left(\varphi_{2}^{-1} \circ \varphi_{1}\right)$.

By Fact 5.6, to obtain permutations with small relative sortedness, it is enough to require that the (sequences of their) corresponding compositions have small monotone subsequences. This method comes with a limitation, as by the Erdös-Szekeres Theorem [ES35], every sequence of $m$ distinct elements contains a monotone subsequence of length $\lceil\sqrt{m}]$. Thus, the smallest relative sortedness that we can achieve between two permutations using Fact 5.6 is $\Theta(\sqrt{m})$. [GS05] showed that, in fact, a very simple permutation has asymptotically optimal relative sortedness with the identity permutation.

Fact 5.7 (Lemma 6 in [GS05]). For every $m$, there exists a permutation $\psi_{m}^{*} \in S_{m}$ such that relsort $\left(\mathrm{id}, \psi_{m}^{*}\right) \leq 2 \cdot \sqrt{m}-1$. Furthermore, $\psi_{m}^{*}$ can be computed in space $O(\log m)$. 
[BHN08] used a simple counting argument to show that a similar bound, still asymptotically optimal, can be achieved even for sets of permutations.

Fact 5.8 (Corollary 2.2 in [BHN08]). Let $k=k(m)$ be a function such that $k \leq m^{O(1)}$. There exists a family $\Phi^{*}=\left(\Phi_{k, m}^{*}\right)_{m}$ where $\Phi_{k, m}^{*}=\left\{\varphi_{1}, \ldots, \varphi_{k}\right\}$ is a set of $k$ permutations from $S_{m}$, such that relsort $\left(\Phi_{k, m}^{*}\right) \leq O(\sqrt{m})$.

Permuted Functions. Let $f=f_{k, n}:\left(\{0,1\}^{n}\right)^{k} \rightarrow\{0,1\}$ be a base function. Let $h=h_{m}:\{0,1\}^{m} \rightarrow\{0,1\}$ be a combining function. Let $\Phi=\Phi_{k, m}=\left\{\varphi_{1}, \ldots, \varphi_{k}\right\}$ be a set of $k$ permutations from $S_{m}$. In what follows, we define $\operatorname{LiftMix}\left(f_{k, n}, h_{m}, \Phi_{k, m}\right)$ : $\left(\{0,1\}^{m \cdot n}\right)^{k} \rightarrow\{0,1\}$ which is a lift-and-mix function that consists of $m$ instances of $f$, permuted by $\Phi$, and combined by $h$.

Let $v \in\left(\{0,1\}^{m \cdot n}\right)^{k}$ be an input. Let $v=\left(v_{1}, \ldots, v_{k}\right)$, where $v_{p} \in\{0,1\}^{m \cdot n}$ for every $p \in[k]$. Let $v_{p}=\left(v_{p, 1}, \ldots, v_{p, m}\right)$, where $v_{p, i} \in\{0,1\}^{n}$ for every $(p, i) \in[k] \times[m]$. Let $v_{p, i}=\left(v_{p, i, 1}, \ldots, v_{p, i, n}\right)$, where $v_{p, i, j} \in\{0,1\}$ for every $(p, i, j) \in[k] \times[m] \times[n]$. When $v$ is given as an input to a TM, $v$ appears on the input tape as

$$
\underbrace{v_{1,1}, \ldots, v_{1, m}}_{v_{1}}, \underbrace{v_{2,1}, \ldots, v_{2, m}}_{v_{2}}, \ldots, \underbrace{v_{k, 1}, \ldots, v_{k, m}}_{v_{k}},
$$

and for $(p, i) \in[k] \times[m], v_{p, i}$ appears as the sequence of bits

$$
v_{p, i, 1}, v_{p, i, 2}, \ldots, v_{p, i, n}
$$

For $\left(p, i^{\prime}\right) \in[k] \times[m]$, we say that $v_{p, \varphi_{p}^{-1}\left(i^{\prime}\right)}$ is the $i^{\prime}$-th instance (of $f$ ) inside $v_{p}$. For $i^{\prime} \in[m]$, we define the $i^{\prime}$-th instance (of $f$ ) inside $v$ to be

$$
v_{\left[i^{\prime}\right], \Phi}:=\left(v_{1, \varphi_{1}^{-1}\left(i^{\prime}\right)}, v_{2, \varphi_{2}^{-1}\left(i^{\prime}\right)}, \ldots, v_{k, \varphi_{k}^{-1}\left(i^{\prime}\right)}\right) \in\left(\{0,1\}^{n}\right)^{k}
$$

For $(p, i) \in[k] \times[m], v_{p, i}$ appears in $v_{\left[i^{\prime}\right], \Phi}$ if and only if $i=\varphi_{p}^{-1}\left(i^{\prime}\right)$. Since this is equivalent to $i^{\prime}=\varphi_{p}(i)$, we observe the following basic fact. 
Fact 5.9. The order on the tape of the $m$ instances inside $v_{p}$ is $\left(\varphi_{p}(1), \varphi_{p}(2), \ldots, \varphi_{p}(m)\right)$, which is precisely seq $\left(\varphi_{p}\right)$. Thus, a left-to-right tape scan over $v_{p}$ visits the $m$ instances in the $\operatorname{order} \operatorname{seq}\left(\varphi_{p}\right)$, and a right-to-left tape scan visits them in the order $\operatorname{seq}\left(\varphi_{p}\right)^{\text {rev }}$.

We now define the lift-and-mix function $\operatorname{LiftMix}(f, h, \Phi):\left(\{0,1\}^{m \cdot n}\right)^{k} \rightarrow\{0,1\}$ by

$$
\operatorname{LiftMix}(f, h, \Phi)(v):=h\left(f\left(v_{[1], \Phi}\right), f\left(v_{[2], \Phi}\right), \ldots, f\left(v_{[m], \Phi}\right)\right) .
$$

We embed an input to $f$ into a specific instance of an input to $F$ as follows. Let $x \in\left(\{0,1\}^{n}\right)^{k}$ be an input to $f$. Let $i^{*} \in[m]$ be an instance number. Let $\bar{y}_{-i^{*}}=$ $\left(y_{1}, \ldots, y_{i^{*}-1}, y_{i^{*}+1}, \ldots, y_{m}\right)$ be a set of $m-1$ inputs to $f$, where $y_{i} \in\left(\{0,1\}^{n}\right)^{k}$ for $i \neq i^{*}$. We define $v_{\Phi}\left(i^{*}, x, \bar{y}_{-i^{*}}\right)$ to be the input to $F$ in which $x$ is embedded at instance $i^{*}$ and $y_{i}$ is embedded at instance $i$, for $i \neq i^{*}$. Formally,

$$
v_{\Phi}\left(i^{*}, x, \bar{y}_{-i^{*}}\right):=v \in\left(\{0,1\}^{m n}\right)^{k} \text { such that } \begin{cases}v_{[i], \Phi}=x, & i=i^{*} \\ v_{[i], \Phi}=y_{i}, & i \neq i^{*}\end{cases}
$$

Corrupted Instances. Let $M$ be a deterministic SM and $v$ be an input of size $N=k \cdot m$. $n$. We say that instance $i \in[m]$ is corrupted in $v$ on $M$ if there exist players $p_{1} \neq p_{2} \in[k]$ such that, during the run of $M$ on $v$, a symbol is pushed on the stack when the input head is scanning $v_{p_{1}, \varphi_{p_{1}}^{-1}(i)}$ and that symbol is popped when the input head is scanning $v_{p_{2}, \varphi_{p_{2}}^{-1}(i)}$, where $\varphi_{p_{1}}, \varphi_{p_{2}} \in \Phi_{k, m}$. Observe that both strings above are part of $v_{[i], \Phi}$, which is instance $i$ inside $v$.

Let $\operatorname{Bad}(M, v) \subseteq[m]$ be the set of all instances which are corrupted in $v$ on $M$. Observe that this definition implicitly depends on the values of $k, m, n$ and $\Phi$, so we should formally write $\operatorname{Bad}_{k, m, n, \Phi}(M, v)$. We use the shorter form for brevity.

Neutral Element. We say that a combining function $h$ has a neutral element $e \in\{0,1\}$ if for every $b \in\{0,1\}$ and for every $i \in[m]$,

$$
h(\underbrace{e, \ldots, e}_{i-1}, b, \underbrace{e, \ldots, e}_{m-i})=b .
$$


Observe that both OR and XOR have neutral element $e=0$.

\subsection{The Reduction}

Using the definitions in Section 5.2, we can now formally restate our main reduction result.

Theorem 5.2 (Formal statement). Let $k=k(n) \geq 2$ be a non-decreasing function and let $m=m(n) \geq 1$ be an increasing function such that $k \leq m^{O(1)}$. Let $N=N(n):=k \cdot m \cdot n$. Let $s=s(N)$ and $r=r(N)$ be increasing functions. Let $\delta<1 / 2$ be a constant. Let $\Phi=\left(\Phi_{k, m}\right)_{m}$ be a family of permutations, where $\Phi_{k, m}=\left\{\varphi_{1}, \ldots, \varphi_{k}\right\}$ are $k$ permutations from $S_{m}$. Let $d:=k \cdot r \cdot \log r \cdot \operatorname{relsort}\left(\Phi_{k, m}\right) / m$.

Let $f=f_{k, n}$ be a boolean base function and let $h=h_{m}$ be a combining function with a neutral element. Let $F=F_{N}:=\operatorname{LiftMix}\left(f_{k, n}, h_{m}, \Phi_{k, m}\right)$.

Assume there exists a randomized nonuniform SM M for F with space bound s, pass bound $r$ and error $\delta$. Then, there exists a randomized $k$-player NIH communication protocol $P$ for $f$, with cost $O(k \cdot r \cdot \log (k \cdot r) \cdot s)$ and error at most $\delta+O(d)$.

In this section, we prove Theorem 5.2 based on the two Lemmas below, which correspond to claims (i) and (iii) in the outline from Section 5.1. First, we formally define the set of corrupted instances in a particular input. Throughout this section, let $n, k, m, N, s, r, \Phi$ be as in Theorem 5.2.

The following Lemma gives a bound on the number of corrupted instances inside a fixed input. As such, it directly corresponds to Claim (i) from the outline in Section 5.1. As opposed to that claim, the Lemma allows for a growing number of players $k=k(n)$ and for two-way passes over the input tape thanks to the "scrambling" of instances according to the family of permutations $\Phi$. We defer its proof to Section 5.4 . 
Lemma 5.10. Let $M^{\prime}$ be a deterministic nonuniform $S M$ with space bound s and (two-way) pass bound $r$. Let $v^{\prime}$ be an input of size N. Then,

$$
\left|\operatorname{Bad}\left(M^{\prime}, v^{\prime}\right)\right| \leq O\left(k \cdot r \cdot \log r \cdot \operatorname{relsort}\left(\Phi_{k, m}\right)\right) .
$$

The next Lemma corresponds to Claim (iii) from the outline in Section 5.1. It says that if the players in a communication protocol embed their input in a non-corrupted instance, then the protocol can efficiently simulate the SM. We defer its proof to Section 5.5 .

Lemma 5.11. Let $M^{\prime}$ be a deterministic nonuniform $S M$. Let $i^{*} \in[m]$ be an instance number and let $\bar{y}_{-i^{*}}=\left(y_{1}, \ldots, y_{i^{*}-1}, y_{i^{*}+1}, \ldots, y_{m}\right)$ be a set of $m-1$ inputs to $f$. Then, there exists a deterministic $k$-player NIH communication protocol $P^{\prime}=P^{\prime}\left(M^{\prime}, i^{*}, \bar{y}_{-i^{*}}\right)$ such that, on input $x$ :

- if $i^{*} \in \operatorname{Bad}\left(M^{\prime}, v\left(i^{*}, x, \bar{y}_{-i^{*}}\right)\right)$, then $P^{\prime}$ outputs "fail";

- otherwise, $P^{\prime}$ correctly simulates $M^{\prime}$ and outputs $M^{\prime}\left(v\left(i^{*}, x, \bar{y}_{-i^{*}}\right)\right)$;

- the cost of $P^{\prime}$ is $O(k \cdot r \cdot \log (k \cdot r) \cdot s)$.

Proof of Theorem 5.2. For every $q$, let $M_{q}$ denote the deterministic nonuniform SM obtained by running $M$ with random string $q$.

Let $D$ be any distribution on the space $\left(\{0,1\}^{n}\right)^{k}$ of inputs to $f$. Below, we give a randomized protocol $P_{D}$ for $f$ with cost and error as described in Theorem 5.2, but with error computed over the choice of the input $x$ from $D$ and of the random coins $\rho$ in $P$. By standard arguments, we can fix $\rho$ to obtain a deterministic protocol with the same error, but only over the choice of $x$ from $D$. Since such a protocol exists for every distribution $D$, by Yao's min-max principle (e.g., Theorem 3.20 in [KN97]), the conclusion of Theorem 5.2 follows.

Without loss of generality, assume that the neutral element of the combining function $h$ is $e=0$. If instead we have neutral element $e=1$, the same proof applies with the roles of 0 and 1 reversed. 
If $D$ has support only on 0 -inputs or only on 1-inputs, $P_{D}$ is trivial. Otherwise, let $D_{0}$ be the distribution $D$, conditioned on $f(x)=0$.

Consider the following protocol $P_{D}$ :

On input $x$ (where player $p$ gets $x_{p}$ ), and shared random string $\rho$ :

Publicly draw $i^{*}$ uniformly from $[m]$

Publicly draw $\bar{y}_{-i^{*}}=\left(y_{1}, \ldots, y_{i^{*}-1}, y_{i^{*}+1}, \ldots, y_{m-1}\right)$ from $\left(D_{0}\right)^{m-1}$

Publicly draw $q$ uniformly

Run $P^{\prime}=P^{\prime}\left(M_{q}, i^{*}, \bar{y}_{-i^{*}}\right)$ (from Lemma 5.11) on input $x$, simulating $M_{q}$ on $v\left(x, i^{*}, \bar{y}_{-i^{*}}\right)$

If $P^{\prime}$ outputs "fail", then $P_{D}$ outputs 1 .

Else, $P_{D}$ outputs the same value as $P^{\prime}$, that is, $M_{q}\left(v\left(x, i^{*}, \bar{y}_{-i^{*}}\right)\right)$

Observe that, since the neutral element of $h$ is $e=0$, and since the instances in $\bar{y}_{-i^{*}}$ are drawn from $D_{0}$, we always have $f(x)=F\left(v\left(i^{*}, x, \bar{y}_{-i^{*}}\right)\right)$. Clearly, the cost of $P_{D}$ is equal to the cost of $P^{\prime}$, which is $O(k \cdot r \cdot \log (k \cdot r) \cdot s)$. To argue about the error in $P_{D}$, we define the following events:

$$
\begin{aligned}
& A=A(x, \rho): P_{D} \text { is correct on input } x \text { with coins } \rho \\
& B=B(q, v): M \text { is correct on input } v \text { with coins } q\left(\text { i.e., } M_{q}(v)=F(v)\right) \\
& C=C\left(i^{*}, q, v\right): \text { instance } i^{*} \text { is not corrupted in } v \text { on } M_{q}\left(\text { i.e., } i^{*} \notin \operatorname{Bad}\left(M_{q}, v\right)\right)
\end{aligned}
$$

Our goal is to show $\operatorname{Pr}[\bar{A}] \leq \delta+O(d)$. By Lemma 5.11, the simulation in $P^{\prime}$ fails if and only if $\bar{C}$. Let $\alpha:=\operatorname{Pr}_{x}[f(x)=1]$. We write

$$
\operatorname{Pr}[A]=\alpha \cdot \operatorname{Pr}[A \mid f(x)=1]+(1-\alpha) \cdot \operatorname{Pr}[A \mid f(x)=0] .
$$

For the left term, consider the conditioning $f(x)=1 . P_{D}$ is correct if either the simulation in $P^{\prime}$ fails (and $P_{D}$ correctly outputs 1 ), or the simulation in $P^{\prime}$ does not fail and $M_{q}$ is correct. Thus, $\bar{C} \vee(C \wedge B) \Rightarrow A$, in particular $B \Rightarrow A$, so $\operatorname{Pr}[A \mid f(x)=$ $1] \geq \operatorname{Pr}[B \mid f(x)=1]$. The event $f(x)=1$ is independent of the choice of $q$, and by 
the correctness condition of $M, \forall v, \operatorname{Pr}_{Q}[B(Q, v)] \geq 1-\delta$. Hence, $\operatorname{Pr}[B \mid f(x)=1]=$ $\operatorname{Pr}[B] \geq 1-\delta$.

For the right term, consider the conditioning $f(x)=0$. Then, $P_{D}$ is correct whenever the simulation in $P^{\prime}$ works and $M_{q}$ is correct, thus $B \wedge C \Rightarrow A$, and

$$
\operatorname{Pr}[A \mid f(x)=0] \geq \operatorname{Pr}[B \mid f(x)=0] \cdot \operatorname{Pr}[C \mid B, f(x)=0] .
$$

As before, $\operatorname{Pr}[B \mid f(x)=0] \geq 1-\delta$. Next, $i^{*}$ is clearly statistically independent of $x$ and $q$. Furthermore, under the conditioning $f(x)=0$, we claim that $i^{*}$ is statistically independent from $v$. To see this, notice how the distribution obtained on pairs $\left(i^{*}, v\right)$ in $P_{D}$ is the same as independently choosing $m 0$-inputs from $D_{0}$ and combining them in $v$, and choosing $i^{*}$ uniformly from $[m]$. Thus, in the expression $\operatorname{Pr}\left[C\left(i^{*}, q, v\right) \mid B(q, v), f(x)=\right.$ $0]$, the conditioning depends on $(x, v, q), C$ itself depends on $i^{*}$, and $i^{*}$ is statistically independent from $(x, v, q)$.

By Lemma 5.10,

$$
\forall(q, v), \quad \operatorname{Pr}_{I^{*}}\left[\overline{C\left(I^{*}, q, v\right)}\right] \leq \frac{O\left(k \cdot r \cdot \log r \cdot \text { relsort }\left(\Phi_{k, m}\right)\right)}{m} \leq O(d) .
$$

Then, $\operatorname{Pr}[C \mid B, f(x)=0]=\operatorname{Pr}[C] \geq 1-O(d)$.

Putting everything together, we get

$$
\begin{aligned}
\operatorname{Pr}[A] & =\alpha \cdot \operatorname{Pr}[A \mid f(x)=1]+(1-\alpha) \cdot \operatorname{Pr}[A \mid f(x)=0] \\
& \geq \alpha \cdot(1-\delta)+(1-\alpha) \cdot(1-\delta) \cdot(1-O(d)) \\
& \geq(1-\delta) \cdot(1-O(d))
\end{aligned}
$$

Then, $\operatorname{Pr}[A] \leq \delta+O(d)$.

\subsection{Bounding the Number of Corrupted Instances}

In this Section we formally prove Lemma 5.10, which corresponds to claim (i) in the outline from Section 5.1 . 
Let $n, k, m, N, s, r, \Phi$ be as in Theorem 5.2. Let $M^{\prime}$ be a deterministic nonuniform SM with space bound $s$ and pass bound $r$. Let $v^{\prime}$ be an input of size $N$. We want to show that the number of corrupted instances in $v^{\prime}$ on $M^{\prime}\left(\right.$ that is, $\left.\left|\operatorname{Bad}\left(M^{\prime}, v^{\prime}\right)\right|\right)$ is at most $O\left(k \cdot r \cdot \log r \cdot \operatorname{relsort}\left(\Phi_{k, m}\right)\right)$.

We say that instance $i \in \operatorname{Bad}\left(M^{\prime}, v^{\prime}\right)$ is corrupted by the 4-tuple $\left(l_{1}, l_{2}, p_{1}, p_{2}\right)$ if $l_{1} \leq$ $l_{2}, p_{1} \neq p_{2}$, and this is the lexicographically smallest 4-tuple such that: a symbol is pushed on the stack during pass $l_{1}$ with the input head scanning $v_{p_{1}, \varphi_{p_{1}}^{-1}(i)}$ (which is instance $i$ inside $v_{p_{1}}$ ), and popped from the stack during pass $l_{2}$ with the input head scanning $v_{p_{2}, \varphi_{p_{2}}^{-1}(i)}$ (which is instance $i$ inside $v_{p_{2}}$ ). In what follows, we first consider the question of how many instances can be corrupted by one 4-tuple. Then, we argue that not all 4-tuples can simultaneously corrupt instances.

Fix one 4-tuple $\left(l_{1}, l_{2}, p_{1}, p_{2}\right)$. Let $I \subseteq[m]$ be the set of instances that are corrupted by this 4-tuple and let $t=|I|$. Label the instances in $I$ by $\left(i_{1}, i_{2}, \ldots, i_{t}\right)$, in the order in which they are visited in the pass $l_{1}$ over $v_{p_{1}}$. By Fact 5.9 , we see that, depending on whether the pass $l_{1}$ is left-to-right or right-to-left, $\left(i_{1}, i_{2}, \ldots, i_{t}\right)$ is a subsequence of either seq $\left(\varphi_{p_{1}}\right)$ or seq $\left(\varphi_{p_{1}}\right)^{\text {rev }}$. Furthermore, because of the First-In Last-Out semantics of a stack, the instances in I must be visited in the order $\left(i_{t}, \ldots, i_{2}, i_{1}\right)$ in the pass $l_{2}$ over $v_{p_{2}}$. Then, again by Fact $5.9,\left(i_{t}, \ldots, i_{2}, i_{1}\right)$ is a subsequence of either $\operatorname{seq}\left(\varphi_{p_{2}}\right)$ or seq $\left(\varphi_{p_{2}}\right)^{\text {rev }}$. Therefore, we see that in any case $\left(i_{1}, i_{2}, \ldots, i_{t}\right)$ is a common subsequence of one of $\left\{\operatorname{seq}\left(\varphi_{p_{1}}\right), \operatorname{seq}\left(\varphi_{p_{1}}\right)^{\text {rev }}\right\}$ and one of $\left\{\operatorname{seq}\left(\varphi_{p_{2}}\right), \operatorname{seq}\left(\varphi_{p_{2}}\right)^{\text {rev }}\right\}$. Hence, $t \leq \operatorname{relsort}\left(\varphi_{p_{1}}, \varphi_{p_{2}}\right) \leq \operatorname{relsort}\left(\Phi_{k, m}\right)$.

Next, consider the $r \times r$ matrix $A$, where $A\left[l_{1}, l_{2}\right]:=1$ if there exist $p_{1}, p_{2} \in[k]$ such that some instance is corrupted by the tuple $\left(l_{1}, l_{2}, p_{1}, p_{2}\right)$, and $A\left[l_{1}, l_{2}\right]:=0$ otherwise. Note that $A=0$ under the main diagonal, because for a tuple to corrupt an instance we must have $l_{1} \leq l_{2}$.

Moreover, for $j \geq 1$, consider the diagonal $l_{2}-l_{1}=j$, and two entries on this diagonal that are $j^{\prime}$ cells apart, for $1 \leq j^{\prime}<j$. We claim that we cannot have $A\left[l_{1}, l_{1}+\right.$ 
$j]=1$ and $A\left[l_{1}+j^{\prime}, l_{1}+j+j^{\prime}\right]=1$. Assume this were true. Then, some instance $i$ is corrupted because a symbol is pushed on the stack in the pass $l_{1}$ and popped in the pass $l_{1}+j$, and another instance $i^{\prime}$ is corrupted because a symbol is pushed on the stack in the pass $l_{1}+j^{\prime}$ and popped in the pass $l_{1}+j+j^{\prime}$. But, with these settings, $l_{1}<l_{1}+j^{\prime}<l_{1}+j<l_{1}+j+j^{\prime}$, which contradicts the First-In Last-Out semantics of a stack.

Hence, for $j \geq 1$, the diagonal $l_{2}-l_{1}=j$ can contain at most $r / j 1$ s. In total, we see that $A$ contains at most $O(r \cdot \log r) 1$ s.

Finally, for fixed $l_{1} \leq l_{2}$, consider the $k \times k$ matrix $B=B_{\left(l_{1}, l_{2}\right)}$, where $B\left[p_{1}, p_{2}\right]:=1$ if some instance is corrupted by the tuple $\left(l_{1}, l_{2}, p_{1}, p_{2}\right)$, and $B\left[p_{1}, p_{2}\right]:=0$ otherwise. First, consider the case when both passes $l_{1}$ and $l_{2}$ are left-to-right.

We claim that, for $j^{\prime} \geq 1$, we cannot have $B\left[p_{1}, p_{2}\right]=1$ and $B\left[p_{1}+j^{\prime}, p_{2}+j^{\prime}\right]=1$. Assume this were true. Then, some instance $i$ is corrupted because a symbol is pushed on the stack during the pass $l_{1}$ over $v_{p_{1}}$ and popped during the pass $l_{2}$ over $v_{p_{2}}$, and another instance $i^{\prime}$ is corrupted because a symbol is pushed on the stack during the pass $l_{1}$ over $v_{p_{1}+j^{\prime}}$ and popped during the pass $l_{2}$ over $v_{p_{2}+j^{\prime}}$. But in the pass $l_{1}, v_{p_{1}}$ is visited before $v_{p_{1}+j^{\prime}}$, and in the pass $l_{2}, v_{p_{2}}$ is visited before $v_{p_{2}+j^{\prime}}$. This contradicts the First-In Last-Out semantics of a stack.

Hence, on every diagonal $i_{2}-i_{1}=j$, the matrix $B$ can contain a single 1 . In total, $B$ contains at most $O(k) 1 \mathrm{~s}$. The cases where either pass $l_{1}$ or $l_{2}$ or both are right-toleft are treated similarly (except that we have to count the entries on the top-right to bottom-left diagonals when the directions of the two passes differ).

So, at most $O(k \cdot r \cdot \log r)$ 4-tuples can corrupt instances, and each 4-tuple can corrupt at most relsort $\left(\Phi_{k, m}\right)$ instances. Therefore,

$$
\left|\operatorname{Bad}\left(M^{\prime}, v^{\prime}\right)\right| \leq O\left(k \cdot r \cdot \log r \cdot \operatorname{relsort}\left(\Phi_{k, m}\right)\right) .
$$




\subsection{Simulating a Stack Machine with a Protocol}

In this Section, we prove Lemma 5.11, which corresponds to claim (iii) in the outline from Section 5.1 .

Let $n, k, m, N, f, s, r, \Phi$ be as in Theorem 5.2. Let $M^{\prime}$ be a deterministic nonuniform SM with space bound $s$ and pass bound $r$. Let $i^{*} \in[m]$ and let $\bar{y}_{-i^{*}}$ be a set of $m-1$ inputs to $f$. Our goal is to show that there exists a deterministic $k$-player NIH communication protocol $P^{\prime}=P^{\prime}\left(M^{\prime}, i^{*}, \bar{y}_{-i^{*}}\right)$ that, on input $x$,

- if $i^{*} \in \operatorname{Bad}\left(M^{\prime}, v\left(i^{*}, x, \bar{y}_{-i^{*}}\right)\right)$, then $P^{\prime}$ outputs "fail";

- otherwise, $P^{\prime}$ correctly simulates $M^{\prime}$ and outputs $M^{\prime}\left(v\left(i^{*}, x, \bar{y}_{-i^{*}}\right)\right)$;

- the cost of $P^{\prime}$ is $O(k \cdot r \cdot \log (k \cdot r) \cdot s)$.

Fix an input $x$ and let $v:=v\left(i^{*}, x, \bar{y}_{-i^{*}}\right)$. Observe that the players in $P^{\prime}$ share $i^{*}$ and $\bar{y}_{-i^{*}}$, and their private inputs are $x_{1}, \ldots, x_{k}$. Thus, from the input $v$ to $M^{\prime}$, they each know all instances $i \neq i^{*}$, and from instance $i^{*}$, player $p$ only knows $x_{p}$. Furthermore, observe that the goal of $P^{\prime}$ is not to compute $f$, but rather, to compute the output of $M^{\prime}$ on $v$.

Private Input Symbols. We say that an input symbol from $v$ is private to player $p$ if it's part of $v_{p, \varphi_{p}^{-1}\left(i^{*}\right)}$, which is where $x_{p}$, the input to player $p$, is embedded in $v$. Input symbols that are not private to any player are public.

Private Stack Symbols. Let $\Gamma$ be the sequence of configurations of $M^{\prime}$ on $v$. Consider a configuration $\gamma \in \Gamma$ and look at the contents of the stack in $\gamma$. For every symbol $\xi$ appearing on the stack, we say that the stack symbol $\xi$ is private to player $p$ if the input head was scanning an input symbol private to player $p$ in the configuration prior to the transition in which $\xi$ was pushed on the stack. A stack symbol that is not private to any player is public. 
Input- and Stack-Private Configurations. We say that configuration $\gamma$ is input-private to player $p$ if the input head in $\gamma$ is scanning an input symbol that is private to player $p$. We say that configuration $\gamma$ is stack-private to player $p$ if it is not input-private to player $p$, the transition out of $\gamma$ is a pop transition and the top stack symbol in $\gamma$ is private to player $p$. A configuration that is neither input- nor stack-private is public.

Intuitively, player $p$ is responsible for simulating the transitions out of configurations that are input- or stack-private to itself. Note that, there exists a configuration that is input-private and stack-private to two different players if and only if $i^{*} \in \operatorname{Bad}\left(M^{\prime}, v\right)$.

Hollow View. We say that player $p$ sees a hollow view of the stack in a configuration $\gamma$ if player $p$ knows:

(i) the stack height;

(ii) for every symbol on the stack, whether it is public, or the player to which it is private (without knowing the symbol itself);

(iii) all stack symbols that are public or private to itself; and

(iv) for every $p^{\prime} \neq p$, the top stack symbol in any contiguous zone of symbols private to player $p^{\prime}$.

We say that a player sees a hollow view of the configuration $\gamma$ if it knows the state of the $\mathrm{SM}$, the location of the input head, and it also sees a hollow view of the stack in $\gamma$.

Some Simple Facts. In the protocol $P^{\prime}$, the players simulate $\Gamma$ transition by transition, always using hollow views of the configurations along the way. We observe the following facts.

(1) If a player $p$ sees a hollow view of a configuration $\gamma$, then that player can determine whether $\gamma$ is public, input-private to some player (since it knows the location of the input head), or stack-private. There is a mild subtlety involving 
the latter, specifically, the definition of $\gamma$ being stack-private involves knowing that the transition out of $\gamma$ is a pop transition. To this end, observe that, by virtue of part (iv) of the hollow view of the stack, if $\gamma$ is not input-private, then player $p$ knows the transition out of $\gamma$, even in the case when the top stack symbol is private to another player.

(2) If a player sees a hollow view of a public configuration $\gamma$, then that player can compute a hollow view of the configuration following $\gamma$ (denoted by next $(\gamma)$ ).

(3) As long as $i^{*} \notin \operatorname{Bad}\left(M^{\prime}, v\right)$, if player $p$ sees a hollow view of a configuration $\gamma$ which is input- or stack-private to player $p$, then player $p$ can compute a hollow view of $\operatorname{next}(\gamma)$.

(4) If player $p$ sees a hollow view of a configuration $\gamma$ that is input-private to itself, it can detect whether $\gamma$ is stack-private to another player $p^{\prime} \neq p$.

The Protocol. We now describe the protocol $P^{\prime}$. The sequence $\Gamma$ is split into several contiguous sections, each of which is of exactly one of the following three types:

(a) Public sections. These sections start with some public configuration (not just any), they consist exclusively of public configurations, and they extend up to, and including, the first configuration which is no longer public.

Inductively, by facts (1) and (2) above, if all players have a hollow view of the configuration at the beginning of a public section, they can all simulate the entire public section starting at that configuration. At the end of the section, the players give control of the simulation to that player to which the configuration reached is input- or stack-private. All this is done without any communication.

(b) Input-private sections. Each such section starts with, and contains, a maximal sequence of input-private configurations to some player $p$, and it ends with the single configuration immediately following that sequence. The latter might be 
public, or input-private to a player other than $p$, or stack-private to any player (including $p$ ).

Inductively, by facts (1), (3) and (4) above, if player $p$ sees a hollow view of the configuration at the beginning of a sequence of input-private configurations to itself, it can either detect that instance $i^{*}$ is corrupted, in which case player $p$ aborts the simulation and the protocol $P^{\prime}$ outputs "fail", or simulate the entire section. Let $\gamma^{\prime}$ be the configuration at the end of the section. At this point, player $p$ communicates:

(v) the state in $\gamma^{\prime}$;

(vi) the input head position in $\gamma^{\prime}$;

(vii) the stack height $L^{\prime}$ in $\gamma^{\prime}$;

(viii) the top stack symbol in $\gamma^{\prime}$;

(ix) the lowest stack height $L^{\prime \prime}$ achieved in a configuration between $\gamma$ and $\gamma^{\prime}$; and

(x) the stack symbol at level $L^{\prime \prime}$.

(c) Mixed stack-private and public sections. Each such section begins with a configuration that is stack-private to some player $p$, and contains a mix of configurations that are either stack-private to $p$ or public.

Let $\gamma$ be the configuration at the beginning of such a section. Let $p$ be the player to which it is stack-private, and assume this player has a hollow view of $\gamma$. Let $\gamma^{\prime}$ be the first configuration following $\gamma$ which is either input-private to any player (including $p$ ), or stack-private to a player other than $p$. Inductively, by facts (1), (2) and (3) above, player $p$ can simulate the entire sequence of configurations between $\gamma$ and $\gamma^{\prime}$.

The key technical point of this entire proof is determining the right place where a player stops a privately simulated section of type (c). It turns out that this is 
not $\gamma^{\prime}$ : doing so could result in public symbols being placed on the stack which would only be known to player $p$, unless they would be subsequently communicated, which would affect the cost of the protocol. Furthermore, it turns out it is also a bad idea for player $p$ to end this section at the first public configuration encountered: the subtlety here is that the stack level might go up and down, and each time it goes down a few private stack symbols are being popped from the same contiguous section of private stack symbols. This scenario would hurt the cost of the protocol, for we could only bound the amount of communication in terms of the length of the contiguous zones of private stack symbols, which can be large.

Instead, player $p$, looking at the entire sequence from $\gamma$ to $\gamma^{\prime}$, computes the minimum possible stack level for this section, and finds the last configuration $\gamma^{\prime \prime}$ preceding, and possibly equal to, $\gamma^{\prime}$ where the stack level is minimal. At this point, player $p$ communicates (v)—(viii) for configuration $\gamma^{\prime \prime}$. Observe that, by definition of $\gamma^{\prime \prime}$, (ix) and (x) are equal to (vii) and (viii), respectively.

Having defined the types of simulated sections, we show that the players have enough information to carry out the simulation. Let $A$ be the number of different sections in the simulation. We claim that for every $1 \leq a \leq A$, all players eventually see a hollow view of the configuration at the beginning of section $a$. We prove this by induction on $a$.

For $a=1$, observe that the initial configuration is either public or input-private to player 1. In either case, all players see a hollow view of the stack, because the stack is empty.

Inductively, assume all players have a hollow view of the configuration $\gamma$ at the beginning of section $a \geq 1$.

If the section is of type (a), by fact (2), all players see a hollow view of the configuration at the end of this section. 
If the section is of type (b), let $p$ be the player to which $\gamma$ is input-private. Clearly, by fact (3), $p$ itself sees a hollow view of the configuration $\gamma^{\prime}$ at the end of the section. After $p$ communicates $(\mathrm{v})-(\mathrm{x})$, each player $p^{\prime} \neq p$ updates its view of the stack as follows: it truncates the stack at height $L^{\prime \prime}$; fills it to the height $L^{\prime}$ with symbols private to player $p$; to compute part (iv) of the hollow view, player $p^{\prime}$ obtains the top stack symbol in $\gamma^{\prime}$ from (ix), and the stack symbol at level $L^{\prime \prime}$ from (x). Taking into account (v) and (vi), now player $p^{\prime}$ sees a hollow view of $\gamma^{\prime}$.

If the section is of type (c), let $p$ be the player to which $\gamma$ is stack-private. Let $\gamma^{\prime \prime}$ be the configuration where this section ends. Clearly, by facts (2) and (3), player $p$ sees a hollow view of $\gamma^{\prime \prime}$. After $p$ communicates (v)-(viii) at the end of the section, each player $p^{\prime} \neq p$ updates its view of the stack as follows: it truncates the stack at height $L^{\prime}$; and it updates the top stack symbol using (vii). Taking into account (v) and (vi), now $p^{\prime}$ sees a hollow view of $\gamma^{\prime \prime}$.

This completes the description of the protocol $P^{\prime}$. If $i^{*} \notin \operatorname{Bad}\left(M^{\prime}, v\right)$, the players get to the final configuration, obtaining the output of $M^{\prime}$ on input $v$. If $i^{*} \in \operatorname{Bad}\left(M^{\prime}, v\right)$, one of the players detects this during a section of type (b), the simulation is aborted, and $P^{\prime}$ and outputs "fail".

The Cost of the Protocol. We now compute the amount of communication in $P^{\prime}$. The number of sections of type (b) is $k \cdot r$, one for every player, in every pass. For $1 \leq a \leq k \cdot r$, let $S_{a}$ be the $a$-th section of type (b) and let $\gamma_{a}$ and $\gamma_{a}^{\prime}$ be the configurations at the beginning and at the end of $S_{a}$.

Fix $a<k \cdot r$, and look at the stack in $\gamma_{a}^{\prime}$. It contains public stack symbols, and several contiguous zones of private stack symbols, at most one such zone for every previous section of type (b). Furthermore, and crucially, observe that:

Claim 5.12. The number of sections of type (c) in between $S_{a}$ and $S_{a+1}$ equals the number of different contiguous zones of private stack symbols from $\gamma_{a}^{\prime}$ (the end of $S_{a}$ ) from which a 
symbol is popped before $\gamma_{a+1}$ (the beginning of $S_{a+1}$ ).

Proof of Claim 5.12. This critical property follows from the way sections of type (c) are terminated. Specifically, we claim it is impossible for two different sections of type (c) between $S_{a}$ and $S_{a+1}$ to pop symbols from the same contiguous zone of stack symbols private to some player $p$.

Assume this was the case for some sections $S_{1}^{\prime}$ and $S_{2}^{\prime}$. In between them, there can be no input-private configurations (by definition of $S_{a}$ and $S_{a+1}$ ), and also no configurations stack-private to a player other than $p$ (otherwise, the zones of private stack symbols corresponding to $S_{1}^{\prime}$ and $S_{2}^{\prime}$ would not be contiguous). Hence, all configurations between $S_{1}^{\prime}$ and $S_{2}^{\prime}$ are either public, or stack-private to player $p$. Moreover, the stack level in $S_{2}^{\prime}$ is strictly lower than in $S_{1}^{\prime}$. Then, player $p$ should not have ended section $S_{1}^{\prime}$ so early, instead, it should have simulated the entire section of configurations between $S_{1}^{\prime}$ and $S_{2}^{\prime}$, and then it should have continued with $S_{2}^{\prime}$.

Clearly, for every contiguous zone of private stack symbols from which symbols are popped in between $S_{a}$ and $S_{a+1}$, there must be at least one associated section of type (c). By the argument above, there will be exactly one such section of type (c).

Let $\gamma_{k r+1}$ denote the final configuration. Consider the $(k \cdot r) \times(k \cdot r)$ matrix $\mathrm{B}$, where $B\left[a_{1}, a_{2}\right]:=1$ if, in between $\gamma_{a_{2}}^{\prime}$ (the end of $S_{a_{2}}$ ) and $\gamma_{a_{2}+1}$ (the beginning of $S_{a_{2}+1}$ or the final configuration), a private stack symbol is popped that was pushed in $S_{a_{1}}$; and $B\left[a_{1}, a_{2}\right]:=0$ otherwise.

Claim 5.13. There are at most $O(k \cdot r \cdot \log (k \cdot r)) 1$ entries in $B$.

Proof of Claim 5.13. The matrix is 0 under the main diagonal, because for a symbol from $S_{a_{1}}$ to be popped after $S_{a_{2}}$, we must have $a_{1} \leq a_{2}$. Consider the diagonal $a_{2}-$ $a_{1}=j$ for some $j \geq 1$. We claim that it is impossible to have $B[a, a+j]=1$ and $B\left[a+j^{\prime}, a+j+j^{\prime}\right]=1$ for some $a$ and some $0<j^{\prime} \leq j$. Assume $B[a, a+j]=1$, so in between $\gamma_{a+j}^{\prime}$ and $\gamma_{a+j+1}$ a symbol is popped that was pushed in $S_{a}$. But then, the 
stack in $\gamma_{a+j+1}$ can no longer contain any symbols pushed in $S_{a^{\prime}}$, for any $a^{\prime}$ such that $a<a^{\prime} \leq a+j$. In particular, for $a^{\prime}=a+j^{\prime}$, all symbols pushed in $S_{a+j^{\prime}}$ are no longer on the stack in $\gamma_{a+j+1}$, which precedes, or equals, $\gamma_{a+j+j^{\prime}}$, Hence, it is impossible to have $B\left[a+j^{\prime}, a+j+j^{\prime}\right]=1$.

The argument above shows that the matrix $B$ contains at most $k \cdot r /(j+1) 1$ entries on the diagonal $a_{2}-a_{1}=j$. Hence, in total, it contains at most $O(k \cdot r \cdot \log (k \cdot r)) 1$ entries.

Lastly, we observe the following.

Claim 5.14. The number of sections of type (c) in between $S_{a}$ and $S_{a+1}$ is at most the number of 1 entries in column a of matrix $B$.

Proof of Claim 5.14. By Claim 5.12, every section of type (c) in between $S_{a}$ and $S_{a+1}$ is associated with a contiguous zone of stack symbols from $\gamma_{a}^{\prime}$ (the end of $S_{a}$ ) that are private to some player $p$. Since all private stack symbols pushed in $S_{a^{\prime}}$ are contiguous on the stack (this is true at any point of the simulation), we see that there can be at most one section of type (c) for every section $S_{a^{\prime}}$ such that symbols from $S_{a^{\prime}}$ are popped in between $S_{a}$ and $S_{a+1}$, or, equivalently, such that $B\left[a^{\prime}, a\right]=1$.

Putting everything together, first observe that sections of type (a) do not result in any communication. There are $k \cdot r$ sections of type (b), and by Claims 5.13 and 5.14 , there are a total of $O(k \cdot r \cdot \log k r)$ sections of type (c).

And the end of every section of type (b) or (c), items (v)-(x) are communicated. The full state takes $O(s)$ bits, the input head position takes $\log N \leq O(s)$ bits, the stack height takes $O(s)$ bits by Fact 5.5. Thus, every communication consists of $O(s)$ bits. The total cost of the protocol $P^{\prime}$ is thus $O(k \cdot r \cdot \log k r \cdot s)$. 


\subsection{Consequences of the Reduction}

In this section, we prove Corollaries 5.3 and 5.4 .

Corollary 5.3 (Restated). Let $\epsilon>0$ and $\delta<1 / 2$ be constants. There exists a constant $\alpha=\alpha(\gamma)>0$ such that the following holds. Let $m=m(n):=n^{\alpha}$, let $k=k(n):=2$, and let $N:=2 \cdot m \cdot n$. Let $\Psi_{2, m}^{*}:=\left\{\mathrm{id}, \psi_{m}^{*}\right\}$ for the permutations $\psi_{m}^{*}$ defined in Fact 5.7. Let $F=F_{N}:=\operatorname{LiftMix}\left(\mathrm{IP}_{2, n}, \mathrm{XOR}_{m}, \Psi_{2, m}^{*}\right)$.

Every randomized $S M$ that computes $F$ with error $\delta$ requires space $s=\omega\left(N^{1 / 4-\epsilon}\right)$ or two-way passes $r=\omega\left(N^{1 / 4-\epsilon}\right)$.

Before going into the proof, observe that $F_{N}$ is a permuted inner product function between two $N$ /2-bit strings. Furthermore, by Fact 5.7, $F_{N}$ is computable in space $\mathrm{O}(\log N)$. Hence, $F \in \mathrm{L}$.

Proof of Corollary 5.3. This is mostly an exercise in setting parameters. If $\epsilon \geq 1 / 4$, there is nothing to prove. Otherwise, let $\beta:=1 / 4-\epsilon>0$. Since $0<\beta<1 / 4$, we get

$$
0<\frac{1}{1-2 \beta}<\frac{1}{2 \beta} \text {. }
$$

Define $\alpha$ so that $\alpha+1$ is in between the two quantities above. That is,

$$
\alpha:=\left(\frac{1}{1-2 \beta}+\frac{1}{2 \beta}\right) \cdot \frac{1}{2}-1 .
$$

Then, $1 /(1-2 \beta)-1<\alpha$ and $\alpha<1 /(2 \beta)-1$. By algebra, these inequalities imply:

$$
\begin{aligned}
& \beta(\alpha+1)<\alpha / 2 \\
& 2 \beta(\alpha+1)<1
\end{aligned}
$$

Assume that there exists a randomized SM $M$ that computes $F$ with error $\delta$ with space bound $s=O\left(N^{\beta}\right)$ and pass bound $r=O\left(N^{\beta}\right)$. By Theorem 5.2 , there exists a randomized communication protocol for $\mathrm{IP}_{2, n}$ with cost $O(k \cdot r \cdot \log (k \cdot r) \cdot s)$ and error at most $\delta+O(d)$, where $d=k \cdot r \cdot \log r \cdot \operatorname{relsort}\left(\Psi_{2, m}^{*}\right) / m$. 
Observe that

$$
\begin{array}{rlrl}
O(d) & =O\left(\frac{k \cdot r \cdot \log r \cdot \operatorname{relsort}\left(\Psi_{2, m}^{*}\right)}{m}\right) & \\
& =O\left(\frac{k \cdot r \cdot \log r}{\sqrt{m}}\right) & & (\text { by Fact 5.7) } \\
& =O\left(\frac{r \cdot \log r}{\sqrt{m}}\right) & & \text { (since } k=2) \\
& =O\left(\frac{(2 \cdot m \cdot n)^{\beta} \cdot \log (2 \cdot m \cdot n)^{\beta}}{\sqrt{m}}\right) & & \left(\text { since } N=2 \cdot m \cdot n \text { and } r=O\left(N^{\beta}\right)\right) \\
& =O\left(\frac{n^{\beta(\alpha+1)} \cdot \log n}{n^{\alpha / 2}}\right) & & \left(\text { since } m=n^{\alpha}\right) \\
& \longrightarrow 0 & & \text { (by Equation (5.1)) }
\end{array}
$$

Hence, eventually, $d<(1 / 2-\delta) / 2$, and the error in $P$ becomes at most $\delta+(1 / 2-$ $\delta) / 2<1 / 2$. By standard arguments, repeating $P$ a constant number of times and taking the majority vote, we get a protocol for $\mathrm{IP}_{2, n}$ with error at most $1 / 3$.

Similarly, observe that

$$
\frac{O(k \cdot r \cdot \log k r \cdot s)}{n}=\frac{O\left((m \cdot n)^{2 \beta} \cdot \log n\right)}{n}=\frac{O\left(n^{2 \beta(\alpha+1)} \cdot \log n\right)}{n} \longrightarrow 0,
$$

where in the limit we use Equation (5.2). Therefore, the cost of the protocol $P$ for $\mathrm{IP}_{2, n}$ (even after being repeated a constant number of times), is $o(n)$. This contradicts the known communication complexity lower bound $R_{2}\left(\mathrm{IP}_{2, n}\right) \geq \Omega(n)$ [CG88].

In order to prove Corollary 5.4, we use the following result, saying that an efficient SM $A$ computing frequency moments can be transformed into an efficient SM $B$ computing the permuted Set Intersection function $F_{N}=\operatorname{LiftMix}\left(\operatorname{pSetInt}_{k, n^{\prime}}, \mathrm{OR}_{m}, \Phi_{k, m}\right)$, for any permutation family $\Phi=\left(\Phi_{k, m}\right)_{m}$. Subsequently, we apply Theorem 5.2 to obtain an efficient NIH protocol for the promise Set Intersection function pSetInt ${ }_{k, n}$, for which we have the lower bound $\Omega(n / k)$ [Gro09].

The basic idea is the following. Let $v$ be an input to $F_{N}$. We construct a sequence $\bar{a}$ on which we eventually run the SM $A$ that computes $\operatorname{Freq}_{\ell}(\bar{a})$. If $v$ contains a 1 bit 
in the part of the input corresponding to player $p \in[k]$, instance $i \in[m]$, and position $j \in[n]$, we add the value $(i-1) \cdot n+j \in[m \cdot n]$ to the sequence $\bar{a}$. The key idea is that, because of the promise inside each instance of pSetInt:

- if $F(v)=0$, the values in $\bar{a}$ are distinct; and

- if $F(v)=1$, some value in $\bar{a}$ appears exactly $k$ times.

By setting the right parameters, the approximate value of $\operatorname{Freq}_{\ell}(\bar{a})$ can be used to distinguish between these cases, thus computing F. As we can see, the stack is not involved at all in the translation of the input, so $B$ is free to use its stack to simulate the stack of $A$.

This streaming reduction originates in [AMS99], where there are no permutations $\Phi$ to deal with. It was rewritten in [BHN08] to deal with the case of several external tapes, where the permutations $\Phi$ are needed. The version in here is a combination of the two: we need to deal with permutations, and we also have to perform it "online", because we have no external tapes in this model. In order to deal with the permutations $\Phi$, we use non-uniformity.

Lemma 5.15. Let $\ell>1, \epsilon \geq 0$ and $0<\delta<1 / 2$ be constants. Let $n, k, m, N, s, r, \Phi$ be as in Theorem 5.2. Set $k=k(n):=\Theta\left((m \cdot n)^{1 / \ell}\right)$. Let $F_{N}:=\operatorname{LiftMix}\left(\operatorname{pSetInt}_{k, n}, \mathrm{OR}_{m}, \Phi_{k, m}\right)$. Assume there exists a randomized SM A with space bound s, pass bound $r$, and error $\delta$, that computes a $(1+\epsilon)$ multiplicative approximation of $\mathrm{Freq}_{\ell}$. Then, there exists a randomized nonuniform $S M B=\left(B_{N}\right)$ that computes $F_{N}$ with space bound $s^{\prime}(N)=s(N)+O(\log N)$, pass bound $r^{\prime}(N)=r(N)+2$, and error $\delta$.

Proof of Lemma 5.15. Let $v$ be an input of size $N$ to $B_{N}$. First, we assume $B_{N}$ has another special tape on which it can write an input to $A$ and then simulate $A$. We describe what this input $\bar{a}=\bar{a}(v)$ is, and we show that $B_{N}$ can compute $F(v)$ from the output of $A$ on $\bar{a}$. Then, we explain how to deal with the fact that $B_{N}$ has no extra tape, and the construction of $\bar{a}$ and the simulation of $A$ on $\bar{a}$ have to be performed in parallel. 
The SM $B_{N}$ begins its computation by counting, in one pass, the number of $1 \mathrm{~s}$ in $v$, and saving this number as the value $C$ on $\log N$ bits of its work tape. In pass number $2, B_{N}$ returns the input head position to the left end of the tape.

We think of the input to each instance of pSetInt $_{k, n}$ as consisting of $n$ columns and $k$ rows. The output is 1 if there exists an all- 1 column, and 0 otherwise. By the promise, we know the number of $1 \mathrm{~s}$ in each column is either 0,1 or $k$, and also that there is at most one all-1 column in each instance of pSetInt $_{k, n}$ We think of $v$, the input to $F_{N}$, as consisting of $m \cdot n$ columns, $n$ of them for each of the $m$ instances.

If $C<k$, there cannot be an all- 1 column in any of the $m$ instances of pSetInt $_{k, n}$, so $B_{N}$ simply outputs 0 . Also, if $C>m \cdot n$, there must be at least 21 s in some column of one one of the $m$ instances of pSetInt $_{k, n}$ (there are only $m \cdot n$ columns in total). In this case, by the promise in pSetInt, that is an all- 1 column, so $B_{N}$ outputs 1 . From now on, assume neither of the above easy cases occurs, so $k \leq C \leq m \cdot n$.

Next, $B_{N}$ constructs an input $\bar{a}$ to $A$ as follows. The bits in $v$ are ordered first by player number $p \in[k]$, then by part $i \in[m]$ (recall, the $m$ instances in $v_{p}$ occur in the $\left.\operatorname{order}\left(\varphi_{p}(1), \ldots, \varphi_{p}(m)\right)\right)$, and finally by bit position $j \in[n]$.

For every $(p, i, j)$ such that $v_{p, i, j}=1$,

$B_{N}$ adds an element with value $\operatorname{val}(i, j):=\left(\varphi_{p}^{-1}(i)-1\right) \cdot n+j$ to $\bar{a}$.

The property of this construction is that the values corresponding to the 1s from the same column of $v$ are all equal, and different from the values corresponding to any other $1 \mathrm{~s}$.

We assume $B_{N}$ is given $\varphi_{1}^{-1}, \ldots, \varphi_{k}^{-1} \in S_{m}$ as non-uniform advice, i.e., it is hardcoded in the state transition of $B_{N}$. To see this is possible without affecting the space bound of $B_{N}$, observe that the input for this computation is $(p, i) \in[k] \times[m]$ and the output is $\varphi_{p}^{-1}(i) \in[m]$. Thus, $O(\log N)$ bits are enough to describe both an input and an output as part of a state of $B_{N}$. We have already assumed that the space bound is always at least $s(N) \geq \Omega(\log N)$. 
Next, observe that $\bar{a}$ consists of exactly $C$ numbers from $[m \cdot n]$, so it is encoded on $N^{\prime}=O(C \cdot \log m n) \leq O(m \cdot n \cdot \log m n)=o(N)$, because $N=k \cdot m \cdot n$ and $k=$ $\Theta\left((m \cdot n)^{1 / \ell}\right)$. Hence, eventually, $N^{\prime} \leq N$.

After constructing $\bar{a}, B_{N}$ runs $A=A_{N^{\prime}}$ on input $\bar{a}$. If $A_{N^{\prime}}$ makes an error, which happens with probability $\delta$, so does $B_{N}$. Otherwise, $A_{N^{\prime}}$ outputs some value

$$
V \in\left[\frac{1}{1+\epsilon} \cdot \operatorname{Freq}_{\ell}(\bar{a}),(1+\epsilon) \cdot \operatorname{Freq}_{\ell}(\bar{a})\right] .
$$

At this point, $B$ outputs 0 if and only if $V \leq C \cdot(1+\epsilon)$, and $B$ outputs 1 otherwise. We need to argue $B$ is correct.

If $F(v)=0$, then all $m$ instances of pSetInt $_{k, n}$ inside $v$ are 0 -instances, so, by the promise, each column has at most one 1 . Hence, all numbers in $\bar{a}$ are distinct, and Freq $_{\ell}(\bar{a})=C$. On the other hand, if $F(v)=1$, then there are $b \geq 1$ 1-instances in $v$ and $m-b 0$-instances. Again by the promise, of all $m \cdot n$ columns in $v, b$ of them have $k 1 \mathrm{~s}$, and all others have at most one 1 . Then, $\operatorname{Freq}_{\ell}(\bar{a})=C-b \cdot k+b \cdot k^{\ell} \geq C+k^{\ell}-k$. Furthermore, if $C \cdot(1+\epsilon)<\left(C+k^{\ell}-k\right) /(1+\epsilon)$, the ranges of possible output values $V$ of $A_{N^{\prime}}$ are disjoint between the cases $F(v)=0$ and $F(v)=1$. Thus, $B$ is correct to output 0 if and only if $V \leq C \cdot(1+\epsilon)$.

We see that the ranges are disjoint if and only if $k^{\ell}-k>\epsilon \cdot C \cdot(2+\epsilon)$. Since $\ell>1$, for large enough $k, k^{\ell}-k>k^{\ell} / 2$. We also know that $m \cdot n \geq C$. Thus, it is enough to have $k^{\ell}>2 \cdot \epsilon \cdot m \cdot n \cdot(2+\epsilon)$, which we achieve by setting $k:=\Theta\left((m \cdot n)^{1 / \ell}\right)$. This completes the argument that $B_{N}$ is correct.

Lastly, we turn to the issue that $B_{N}$ doesn't have an extra tape to first write $\bar{a}$ and then simulate $A_{N^{\prime}}$ on $\bar{a}$, but has to do these in parallel. To this end, $B_{N}$ operates as follows. At every step, it keeps the three values $(p, i, j) \in[k] \times[m] \times[n]$, corresponding to the location of the input head in $v$, using $\log N$ bits on its work tapes. $B_{N}$ also has a buffer of $\log (m \cdot n) \leq \log N$ bits, which it fills with the value $\operatorname{val}(i, j) \in[m \cdot n]$. $B_{N}$ simulates $A_{N^{\prime}}$ by using the buffer as the input tape of $A_{N^{\prime}}$, using space $s\left(N^{\prime}\right)$ on its work tapes, and using its own stack as the stack of $A_{N^{\prime}}$. At any point where 
$A_{N^{\prime}}$ attempts to move the head left (respectively, right) and the corresponding value from $\bar{a}$ is not in the buffer, $B_{N}$ pauses the simulation of $A_{N^{\prime}}$, scans left (respectively, right) on its input tape until it finds the next 1 bit, and refills the buffer with the new value val $(i, j)$. Observe that $B_{N}$ uses at most as many passes as $A_{N^{\prime}}$ during this simulation. In total, $B_{N}$ makes at most $r\left(N^{\prime}\right)+2 \leq r(N)+2$ passes, and uses space $s\left(N^{\prime}\right)+O(\log N) \leq s(N)+O(\log N)$.

We are now in a position to prove the trade-off lower bound for computing frequency moments with a SM.

Corollary 5.4(Restated). Let $\ell>4, \epsilon \geq 0$ and $\delta<1 / 2$ be constants. There exists a constant $0<\beta<1$ such that any randomized SM computing $a(1+\epsilon)$ multiplicative approximation of Freq $_{\ell}$ with error $\delta$ requires space $s=\omega\left(N^{\prime \beta}\right)$ or passes $r=\omega\left(N^{\prime} \beta\right)$, where $N^{\prime}$ denotes the input size.

Proof of Corollary 5.4. We set the parameters first. Let $\beta:=(\ell-4) /(5 \cdot(\ell+1))$. Observe that $0<\beta<(\ell-4) /(4 \cdot(\ell+1))<1$. By algebra, this implies

$$
0<\frac{1}{1-2 / \ell-2 \beta(1+1 / \ell)}<\frac{1}{2 / \ell+2 \beta(1+1 / \ell)}
$$

Set $\alpha$ so that $\alpha+1$ is halfway in between the two quantities above, that is,

$$
\alpha:=\left(\frac{1}{1-2 / \ell-2 \beta(1+1 / \ell)}+\frac{1}{2 / \ell+2 \beta(1+1 / \ell)}\right) \cdot \frac{1}{2}-1
$$

Thus,

$$
\frac{1}{1-2 / \ell-2 \beta(1+1 / \ell)}<\alpha+1<\frac{1}{2 / \ell+2 \beta(1+1 / \ell)} .
$$

By algebra, the two inequalities above imply:

$$
\begin{aligned}
(1+\alpha) \frac{1}{\ell}+(1+\alpha) \beta\left(1+\frac{1}{\alpha}\right) & <\frac{\alpha}{2} \\
2(1+\alpha) \frac{1}{\ell}+2(1+\alpha) \beta\left(1+\frac{1}{\alpha}\right) & <1
\end{aligned}
$$


Assume there exists a SM $A$ approximating Freq $q_{\ell}$ to within $(1+\epsilon)$, that has space bound $s\left(N^{\prime}\right)=O\left(N^{\prime} \beta\right)$, pass bound $r\left(N^{\prime}\right)=O\left(N^{\prime} \beta\right)$, and error $\delta$. We derive a contradiction.

Set $m:=n^{\alpha}$ and $k=k(n):=\Theta\left((m \cdot n)^{1 / \ell}\right)=\Theta\left(n^{(1+\alpha) / \ell}\right)$. Set $\Phi_{k, m}:=\Phi_{k, m^{\prime}}^{*}$ for the family of permutations $\Phi_{k, m}^{*}$ defined in Fact 5.8. By Lemma 5.15, there exists a randomized nonuniform SM $B=B_{N}$ for $F_{N}=\operatorname{LiftMix}\left(\operatorname{pSetInt}_{k, n^{\prime}} \mathrm{OR}_{m}, \Phi_{k, m}^{*}\right)$ with space bound $s(N)=O\left(N^{\beta}\right)$, pass bound $r(N)=O\left(N^{\beta}\right)$, and error $\delta$. By Theorem 5.2, there exists a randomized NIH communication protocol $P$ for pSetInt ${ }_{k, n}$ with $\operatorname{cost} O(k \cdot r \cdot \log (k \cdot r) \cdot s)$ and error $\delta+O(d)$ where $d=k \cdot r \cdot \log r \cdot \operatorname{relsort}\left(\Phi_{k, m}^{*}\right) / m$.

Observe that

$$
\begin{array}{rlr}
O(d) & =O\left(\frac{k \cdot r \cdot \log r \cdot \text { relsort }\left(\Phi_{k, m}^{*}\right)}{m}\right) & \\
& =O\left(\frac{k \cdot r \cdot \log r}{\sqrt{m}}\right) & (\text { by Fact 5.8) } \\
& =O\left(\frac{n^{(1+\alpha) / \ell} \cdot n^{(1+\alpha)(1+1 / \ell) \beta} \cdot \log n}{n^{\alpha / 2}}\right) & \left(\text { using } r=O\left((k \cdot m \cdot n)^{\beta}\right)\right. \text { and replacing) } \\
& \longrightarrow 0 & \text { (by Equation (5.3)) }
\end{array}
$$

Thus, eventually $d<(1 / 2-\delta) / 2$, and the error in $P$ becomes at most $\delta+(1 / 2-$ $\delta) / 2<1 / 2$. By standard arguments, repeating $P$ a constant number of times and taking the majority vote we get a similar protocol with error at most $1 / 3$.

Furthermore, observe that

$$
\frac{O(k \cdot r \cdot \log (k \cdot r) \cdot s)}{n / k}=\frac{O\left(n^{2(1+\alpha) / \ell} \cdot n^{2(1+\alpha)(1+1 / \ell) \beta} \cdot \log n\right)}{n} \longrightarrow 0,
$$

where we first used $r, s \leq(k \cdot m \cdot n)^{\beta}$, replaced for $n$, and finally used Equation (5.4). Therefore, the cost of $P$, even after constantly many repetitions, is $o(n / k)$. This contradicts the known lower bound $R_{\mathrm{NIH}, k}\left(\right.$ pSetInt $\left._{k, n}\right) \geq \Omega(n / k)$ [Gro09]. 


\subsection{Discussion}

There are several known reductions between efficient communication protocols and various types of small space Turing Machines. In this chapter, we added to this arsenal a new reduction showing that an efficient communication protocol can simulate a Turing Machine with small space, few passes, and an unbounded stack.

To obtain this reduction, we adapted a framework presented in [BHN08]. Starting with a $(k \cdot n)$-bit base function $f$, we constructed a lifted $(k \cdot m \cdot n)$-bit function $F$ which consists of $m$ disjoint instances to $f$, glued together by a combining gate, usually the top gate of $f$. Assuming there exists a Stack Machine computing $F$ with small space and few passes, we presented an efficient communication protocol for $f$. The idea is that the players in the protocol construct an input $v$ to $F$ by choosing $m-1$ random "decoy" instances to $f$, and embedding their real input $x$ as the remaining instance. Henceforth, the players simulate the Stack Machine on input $v$, and, if the simulation is successful, they retrieve $f(x)$ from $F(v)$. Intuitively, by hiding the real input instance within a sea of decoy instances, the goal of the players is to "confuse" the Stack Machine into not dedicating its stack to computation on the real instance. We showed that if the Stack Machine is so "confused", the players can simulate it efficiently, because they do not have to communicate stack contents. In the outline Section 5.1, we gave an easy argument showing that a Stack Machine with one-way access to the input is easily "confused". Subsequently, in Section 5.4, we showed that a Stack Machine with two-way access to the input is also "confused" in the way described above if the $m$ instances inside $F$ are "scrambled" by a family of permutations that have small relative sortedness.

Our arguments are similar to the ones in [BHN08] at the general framework level. The trade-off lower bounds they obtained are for "read/write Stream Algorithms"; these are Turing Machines that have internal tapes and a constant number of read- 
write external tapes, space is bounded on the internal tapes, and passes are bounded over all external tapes. There is an "obvious" simulation of a Stack Machine by a r/w Stream Algorithm with 2 external tapes: use the second external tape to simulate the stack. However, if the Stack Machine makes a few passes over its input tape, it does not follow that the $\mathrm{r} / \mathrm{w}$ Stream Algorithm makes a few passes over both of its external tapes. The crucial point is that there is no bound on the number of passes (reversals) the Stack Machine makes on its stack, but such a bound appears in the $\mathrm{r} / \mathrm{w}$ Stream Algorithm simulating the Stack Machine. As a result, this particular simulation cannot be used to obtain trade-off lower bounds for Stack Machines from similar lower bounds for $\mathrm{r} / \mathrm{w}$ Stream Algorithms. In fact, [DPS09, Lemma 12] provided evidence that there does not exists an efficient simulation of a Stack Machine by a $\mathrm{r} / \mathrm{w}$ Stream Algorithm.

Despite its connection to circuit classes, The Stack Machine model of computation is not well understood. We believe it would be interesting to study what problems can actually be solved by efficient Stack Machines.

It would also be interesting to obtain space-time trade-offs for this model, as opposed to the space-passes trade-offs obtained in here. To be more precise, we can simply replace passes by time in our bounds, but, at least in the case of regular Turing Machines, we can obtain better (larger) space-time lower bounds by simple padding arguments (see, for example, Lemma 12.1 in [KN97]). However, no such arguments are known in the case of either Stack Machines or r/w Stream Algorithms. 


\section{Bibliography}

[ABI86] Noga Alon, László Babai, and Alon Itai. A fast and simple randomized parallel algorithm for the maximal independent set problem. Journal of Algorithms, 7(4):567-583, 1986.

[All89] Eric Allender. P-uniform circuit complexity. Journal of the ACM, 36(4):912928, 1989.

[AMS99] Noga Alon, Yossi Matias, and Mario Szegedy. The space complexity of approximating the frequency moments. Journal of Computer and System Sciences, 58(1):137-147, 1999.

$\left[\mathrm{BCD}^{+} 89\right]$ Allan Borodin, Stephen Cook, Patrick Dymond, Larry Ruzzo, and Martin Tompa. Two applications of inductive counting for complementation problems. SIAM Journal on Computing, 18(3):559-578, 1989.

[BDPW07] Paul Beame, Matei David, Toniann Pitassi, and Philipp Woelfel. Separating deterministic from nondeterministic NOF multiparty communication complexity. In International Colloquium on Automata, Languages and Programming, pages 134-145. Springer, 2007.

[BFS86] László Babai, Péter Frankl, and János Simon. Complexity classes in communication complexity theory (preliminary version). In Symposium on the Foundations of Computer Science, pages 337-347. IEEE, 1986. 
[BHK01] László Babai, Thomas P. Hayes, and Peter G. Kimmel. The cost of the missing bit: Communication complexity with help. Combinatorica, 21(4):455$488,2001$.

[BHN08] Paul Beame and Dang-Trinh Huynh-Ngoc. On the value of multiple read/write streams for approximating frequency moments. In Symposium on the Foundations of Computer Science, pages 499-508. IEEE, 2008.

[BHN09] Paul Beame and Dang-Trinh Huynh-Ngoc. Multiparty communication complexity and threshold circuit size of $\mathrm{AC}^{0}$. In Symposium on the Foundations of Computer Science. IEEE, 2009.

[BNS92] László Babai, Noam Nisan, and Márió Szegedy. Multiparty protocols, pseudorandom generators for logspace, and time-space trade-offs. Journal of Computer and System Sciences, 45(2):204-232, 1992.

[BPH09] Paul Beame, Toniann Pitassi, and Trinh Huynh. Hardness amplification in proof complexity. Technical Report TR09-072, Electronic Colloquium on Computational Complexity (ECCC), 2009.

[BPS07] Paul Beame, Toniann Pitassi, and Nathan Segerlind. Lower bounds for Lovász-Schrijver systems and beyond follow from multiparty communication complexity. SIAM Journal on Computing, 37(3):845-869, 2007.

[BT94] Richard Beigel and Jun Tarui. On ACC. Computational Complexity, 4(4):350366, 1994.

[BV02] Paul Beame and Erik Vee. Time-space tradeoffs, multiparty communication complexity, and nearest-neighbor problems. In Symposium on the Theory of Computing, pages 688-697. ACM, 2002. 
[CA08] Arkadev Chattopadhyay and Anil Ada. Multiparty communication complexity of disjointness. Technical Report TR08-002, Electronic Colloquium on Computational Complexity (ECCC), 2008.

[CFL83] Ashok Chandra, Merrick Furst, and Richard Lipton. Multi-party protocols. In Symposium on the Theory of Computing, pages 94-99. ACM, 1983.

[CG88] Benny Chor and Oded Goldreich. Unbiased bits from sources of weak randomness and probabilistic communication complexity. SIAM Journal on Computing, 17(2):230-261, 1988.

[CG89] Benny Chor and Oded Goldreich. On the power of two-point based sampling. Journal of Complexity, 5(1):96-106, 1989.

[Cha07] Arkadev Chattopadhyay. Discrepancy and the power of bottom fan-in in depth-three circuits. In Symposium on the Foundations of Computer Science, pages 449-458. IEEE, 2007.

[CKS03] Amit Chakrabarti, Subhash Khot, and Xiaodong Sun. Near-optimal lower bounds on the multi-party communication complexity of set disjointness. In Conference on Computational Complexity, pages 107-117. IEEE, 2003.

[Coo71] Stephen Cook. Characterizations of pushdown machines in terms of timebounded computers. Journal of the ACM, 18:4-18, 1971.

[CT93] Fan Chung and Prasad Tetali. Communication complexity and quasi randomness. SIAM Journal on Discrete Mathematics, 6(1):110-123, 1993.

[DPS09] Matei David, Periklis Papakonstantinou, and Anastasios Sidiropoulos. Polynomial time with restricted use of randomness. Technical Report TR09-039, Electronic Colloquium on Computational Complexity, 2009. 
[DPV09] Matei David, Toniann Pitassi, and Emanuele Viola. Improved separations between nondeterministic and randomized multiparty communication. ACM Transactions on Computation Theory, 1(2):1-20, 2009.

[ES35] Paul Erdös and George Szekeres. A combinatorial problem in geometry. Compositio Math. 2: 463-470, 1935.

[FG05] Jeff Ford and Anna Gál. Hadamard tensors and lower bounds on multiparty communication complexity. In International Colloquium on Automata, Languages and Programming, pages 1163-1175. Springer, 2005.

[Gro09] Andre Gronemeier. Asymptotically optimal lower bounds on the NIHmulti-party information complexity of the and-function and disjointness. In Symposium on the Theoretical Aspects of Computer Science, pages 505-516, 2009.

[GS05] Martin Grohe and Nicole Schweikardt. Lower bounds for sorting with few random accesses to external memory. In Symposium on the Principles of Database Systems, pages 238-249. ACM, 2005.

[HG91] Johan Håstad and Mikael Goldmann. On the power of small-depth threshold circuits. Computational Complexity, 1(2):113-129, 1991.

[Kla07] Hartmut Klauck. Lower bounds for quantum communication complexity. SIAM Journal on Computing, 37(1):20-46, 2007.

[KN97] Eyal Kushilevitz and Noam Nisan. Communication complexity. Cambridge University Press, 1997.

[KS92] Bala Kalyanasundaram and Georg Schintger. The probabilistic communication complexity of set intersection. SIAM Journal on Discrete Mathematics, 5(4):545-557, 1992. 
[LS08] Troy Lee and Adi Shraibman. Disjointness is hard in the multi-party number-on-the-forehead model. In Conference on Computational Complexity, pages 81-91. IEEE, 2008.

[MNT93] Yishay Mansour, Noam Nisan, and Prasoon Tiwari. The computational complexity of universal hashing. Theoretical Computer Science, 107(1):121133, 1993.

[New91] Ilan Newman. Private vs. common random bits in communication complexity. Information Processing Letters, 39(2):67-71, 1991.

[NN93] Joseph Naor and Moni Naor. Small-bias probability spaces: efficient constructions and applications. SIAM Journal on Computing, 22(4):838-856, 1993.

[NS94] Noam Nisan and Mario Szegedy. On the degree of boolean functions as real polynomials. Computational Complexity, 4:301-313, 1994.

[Pat92] Ramamohan Paturi. On the degree of polynomials that approximate symmetric boolean functions. In Symposium on the Theory of Computing, pages 468-474. ACM, 1992.

[Pud03] Pavel Pudlák. An application of Hindman's theorem to a problem on communication complexity. Combinatorics, Probability and Computing, 12(6):661-670, 2003.

[Raz90] Alexander Razborov. On the distributional complexity of disjointness. In International Colloquium on Automata, Languages and Programming, pages 249-253. Springer, 1990.

[Raz00] Ran Raz. The BNS-Chung criterion for multi-party communication complexity. Computational Complexity, 9(2):113-122, 2000. 
[Raz03] Alexander Razborov. Quantum communication complexity of symmetric predicates. Izvestiya: Mathematics, 67(1):145-159, 2003.

[Ruz80] Walter Ruzzo. Tree-size bounded alternation. Journal of Computer and System Sciences, 21(2):218-235, 1980.

[Ruz81] Walter Ruzzo. On uniform circuit complexity. Journal of Computer and System Sciences, 22(3):365-383, 1981.

[She08a] Alexander Sherstov. Communication lower bounds using dual polynomials. Bulletin of the EATCS, 95:59-93, 2008.

[She08b] Alexander Sherstov. The pattern matrix method for lower bounds on quantum communication. In Symposium on the Theory of Computing, pages 85-94. ACM, 2008.

[She09] Alexander Sherstov. Separating $\mathrm{AC}^{0}$ from depth-2 majority circuits. SIAM Journal on Computing, 38(6):2113-2129, 2009.

[Vee04] Erik Vee. Time-space tradeoffs for nonuniform computation. PhD thesis, University of Washington, 2004.

[VW08] Emanuele Viola and Avi Wigderson. Norms, XOR lemmas, and lower bounds for GF(2) polynomials and multiparty protocols. Theory of Computing, 4:137-168, 2008.

[Yao79] Andrew Yao. Some complexity questions related to distributive computing (preliminary report). In Symposium on the Theory of Computing, pages 209-213. ACM, 1979. 